\title{
Planning for Mars Returned Sample Science: Final Report of the MSR End-to-End International Science Analysis Group (E2E-iSAG)
}

\author{
A report requested by the Mars Exploration Program Analysis Group (MEPAG) November 22, 2011
}

\section{Table of Contents}

Executive Summary 176

1. Introduction 178

1.1. A proposed MSR implementation architecture 180

2. MSR Campaign Scientific Aims and Objectives 180

2.1. Introduction 180

2.2. Specific proposed science aims and objectives for Mars returned sample science (organized by topic) 180

2.2.1. Aim A. Life

2.2.2. Aim B. Surface

2.2.4. Aim D. Prepare for human exploration

2.3. Prioritization of scientific objectives 195

3. Achieving the Scientific Objectives—Some Considerations Involving Collecting Samples from a Field Area 196

3.1. Relationship between field and sample science 196

3.2. Information hierarchy 196

3.3. Collect early, exchange later 196

3.4. The importance of rock and soil sample suites 197

4. Achieving the Proposed Scientific Objectives—Samples Required/Desired 197

4.1. Integrated priorities for rock samples 197

4.1.1. Considerations in designing the suites of rock samples 197

4.1.2. Possible/probable variations in rock we need to be prepared to detect and sample 198

4.1.3. Scientific value of a subsurface rock sample 198

4.1.4. Number of rock samples desired/required 199

4.2. Considerations related to the number and type of regolith samples 199

4.2.2. Regolith—sampling implications 199

4.3. Considerations related to the number and type of gas samples 200

4.3.1. Integrated priorities for gas samples 200

4.3.2. Atmospheric gas—sampling implications 200

4.3.3. The importance of rock/mineral samples with fluid or melt inclusions 200

5. Where on Mars Might It Be Possible to Obtain the Samples Needed to Achieve the Proposed Objectives

5.1. Establishing a reference landing site set 200

5.2. Brief descriptions of the reference landing sites 201

5.3. Implications of the reference landing set for the major EDL and mobility parameters 203

6. Measurements on Returned Samples Required to Achieve the Proposed Objectives 204

6.1. Analysis flow for rock and regolith samples 204

6.1.1. Preliminary examination 204

Recommended bibliographic citation: MEPAG E2E-iSAG. (2011) Planning for Mars returned sample science: final report of the MSR Endto-End International Science Analysis Group (E2E-iSAG). Astrobiology 12:175-230.

Or: McLennan, S.M., Sephton, M.A., Allen, C., Allwood, A.C., Barbieri, R., Beaty, D.W., Boston, P., Carr, M., Grady, M., Grant, J., Heber, V.S., Herd, C.D.K., Hofmann, B., King, P., Mangold, N., Ori, G.G., Rossi, A.P., Raulin, F., Ruff, S.W., Sherwood Lollar, B., Symes, S., and Wilson, M.G. (2011) Planning for Mars returned sample science: final report of the MSR End-to-End International Science Analysis Group (E2E-iSAG). Astrobiology 12:175-230.

Inquiries regarding this report should be directed to Scott McLennan (scott.mclennan@sunysb.edu) or Mark Sephton (m.a.sephton@ imperial.ac.uk), E2E-iSAG co-chairs, or to David Beaty (David.Beaty@jpl.nasa.gov).

JPL Document Review Clearance CL\#11-5351. 
6.1.2. Planetary protection 205

6.1.3. Scientific research 206

6.1.4. Requirement for replicate analyses 206

6.1.5. Reserve samples for future research 207

6.2. Implications for sample sizing: rock samples 207

6.3. Implications for sample sizing: regolith samples 208

6.4. Implications for sample sizing: atmospheric gas samples 208

7. Capabilities on Mars Needed to Select, Acquire, and Preserve the Samples 209

7.1. Observations required to understand geological context 209

7.1.1. Scale of required field observations 210

7.2. The Pasteur payload 211

7.2.1. Potential use of the instruments of the Pasteur payload to support the objectives
of the MSR Campaign

7.3. Measurement needs of the proposed 2018 joint rover in addition to those of Pasteur 212

7.3.1. Mast-mounted instruments 212

7.3.2. Arm-mounted instruments 212

7.3.3. Summary of on-Mars measurement needs in excess of Pasteur 213

7.4. Sample collection and preservation system 213

7.4.1. Sizing the sample cache 213

7.4.2. Sample packing 214

7.4.3. Organic blanks and calibration standards 214

7.4.4. Sample sealing and preservation 215

7.4.5. Sealing concepts and materials 215

8. Conclusions 216

8.1. Summary 216

8.2. Recommendation for future work 217

8.2.1. MEPAG-related tasks 217

8.2.2. Programmatic issues (NASA and ESA) 217

8.2.3. Research work (NASA and ESA) 217

8.2.4. Engineering development (NASA and ESA) 217

A. Appendices 217

A1. Glossary 217

A2. Charter of This Study 218

A2.1. Charter assumptions 218

A2.2. Methodology and approach 218

\begin{tabular}{lr} 
A2.2.1. & Requested tasks \\
\hline A2.2.
\end{tabular}

$\begin{array}{lr}\text { A2.2.2. Methods } & 219\end{array}$

A2.2.3. Deliverables, schedule
219

A3. Scientific Risk for the MSR Campaign 219

A4. Planetary Protection Issues/Opportunities 220

A5. Reference Landing Sites 221

Acknowledgments 221

Abbreviations 221

References 222

\section{Executive Summary}

$\mathbf{R}$ ETURNing SAMPLes from Mars to Earth for scientific analysis has been, and continues to be, among the highest-priority objectives of planetary science. Partly for this reason, the 2011 Planetary Science Decadal Survey placed high priority on a proposed 2018 rover mission that would conduct careful in situ science and use that scientific information to select and cache samples that could be returned to Earth by a potential future mission. To ensure that the potential contributions of the 2018 rover to the proposed Mars Sample Return (MSR) Campaign are properly planned, this study was undertaken to consider the science of the MSR Campaign concept from end to end. This white paper is the principal output of the MSR End-to-End International Science
Analysis Group (E2E-iSAG): a group chartered by the Mars Exploration Program Analysis Group (MEPAG).

We have built upon previous MEPAG and National Research Council (NRC) studies to consolidate and prioritize science objectives for a potential MSR campaign. Considering those objectives, we evaluated the implications for accessing, selecting, obtaining, and caching suitable samples on Mars during the proposed 2018 in situ science rover mission. Key issues addressed include the types of material needed (rock, regolith, gas), the number and character of samples and sample suites, the resulting sample mass, the in situ science measurements needed to establish the geological context of the samples, and the types of landing sites on Mars that could provide the diverse materials needed to meet the science objectives. As one of the key inputs to this analysis, 
we also evaluated the range of likely analytical investigations that would be carried out on the returned samples.

In developing science objectives and priorities for MSR, the E2E-iSAG identified four overarching science themes or Aims:

(A) Life and its organic chemical precursors,

(B) Surface materials and the record of martian surface processes,

(C) Planetary evolution of Mars and its atmosphere, and

(D) Potential for future human exploration.

Within these Aim categories, eight specific scientific objectives were defined that could be addressed through the analysis of returned materials. Using criteria based on the value of increased knowledge that could be gained by analyzing returned samples, we placed the eight objectives in priority order as follows:

(1) Critically assess any evidence for past life or its chemical precursors, and place detailed constraints on the past habitability and the potential for preservation of the signs of life.

(2) Quantitatively constrain the age, context, and processes of accretion; early differentiation; and magmatic and magnetic history of Mars.

(3) Reconstruct the history of surface and near-surface processes involving water.

(4) Constrain the magnitude, nature, timing, and origin of past planet-wide climate change.

(5) Assess potential environmental hazards to future human exploration.

(6) Assess the history and significance of surface modifying processes, including, but not limited to, impact, photochemical, volcanic, and aeolian processes.

(7) Constrain the origin and evolution of the martian atmosphere, accounting for its elemental and isotopic composition with all inert species.

(8) Evaluate potential critical resources for future human explorers.

In addition, evaluating the possibility of extant life in all returned samples would be important, both to meet planetary protection requirements and because of the intrinsic scientific interest. However, we felt that there would be no logical way to implement the search for extant life as a primary mission objective that would be expected to be achieved.

Returned sample types most likely to achieve the objectives described above are, in priority order;

(1A) Suites of subaqueous or hydrothermal sediments (equal priority),

(1B) Suites of hydrothermally altered rocks or lowtemperature fluid-altered rocks (equal priority),

(2) Suite of unaltered igneous rocks,

(3) At least one and preferably two or more samples of regolith, including airfall dust, obtained some distance from any landing site contamination and preferably including a subsurface sample,

(4) At least one and preferably two aliquots of presentday atmosphere and samples of sedimentary-igneous rocks containing ancient trapped atmosphere.

The E2E-iSAG found that the value of returned sample science is dependent on the quality of in situ science. Parti- cularly to address the higher-priority science objectives, sample suites would need to be collected from a site that has been well characterized through a campaign of in situ field science. The goal of site characterization would be the establishment of geological context so that the relationship of samples to each other, and to their surroundings, could be understood. This information would ensure that only the best samples would be returned to Earth and that measurements made on Earth could be confidently interpreted and lead to the most significant discoveries.

To obtain the required context, previous experience demonstrates the need for integrated observations ranging from macroscopic (i.e., regional, outcrop) down to microscopic (i.e., submillimeter) scales. Experience from terrestrial studies and the Mars Exploration Rovers (MERs) further demonstrates the need to evaluate many more rocks and soils than are eventually collected (by several orders of magnitude) and also the need to remove dust and weathering products from rock surfaces in order to interpret the rocks correctly. This characterization would require in situ measurements from outcrops and soils across the areas of interest as well as the precise locations of the samples selected; thus, a suite of scientific instruments and supporting capabilities on the sample-collecting rover would be needed.

To achieve the proposed science objectives, the total number of rock samples should be $\sim 30$. To prepare for new discoveries during surface operations, a capability to exchange $\geq 25 \%$ of earlier collected samples with later collected samples would add valuable scientific flexibility. The $2 \mathrm{~m}$ ESA drill would provide unique sampling opportunities from the unexplored subsurface, with its enhanced likelihood of preservation of organics; accordingly, obtaining these samples is also highly desirable.

To evaluate the size of individual samples needed to meet science objectives, the E2E-iSAG reviewed various analytical methods likely to be applied to returned samples by preliminary examination teams, for planetary protection (i.e., life detection, biohazard assessment), and by principal investigators. The E2E-iSAG concluded that samples should be sized so that all high-priority analyses could be done in triplicate and that at least $40 \%$ of each sample should be preserved for future scientific investigations, consistent with standard curatorial practice of extraterrestrial materials. Samples sized at $15-16 \mathrm{~g}$ would be optimal, and containers designed to accommodate sedimentary and igneous rocks of this mass would also be sufficient for regolith samples. Total mass of returned rocks, soils, blanks, and standards should be $\sim 500 \mathrm{~g}$. To achieve all high-priority objectives related to an atmospheric gas sample, it should be sized at the equivalent of $50 \mathrm{~cm}^{3}$ at Mars ambient atmospheric conditions (which is equivalent to $5 \mathrm{~cm}^{3}$ with $10 \times$ compression).

To preserve acceptable sample quality during storage on Mars-perhaps for many years-and to transport the cache to Earth, the sample containers would need to be sealed. The critical volatile component to be considered in devising containment is structural and adsorbed water that may be present in some samples. Accordingly, individual sample tubes would require some level of sealing during storage on the martian surface. It would also be scientifically desirable to seal the entire sample canister before leaving Mars to avoid a significant pressure differential across sample tube seals during transit and thus minimize volatile mobility. 
Finding sites that would contain the desired samples and also be safe to land on is challenging. To overcome this challenge, it may be necessary to have sufficient mobility to explore outside landing ellipses and the capability to avoid or tolerate certain hazards during entry, descent, and landing (EDL) so that the ellipse may include rocky materials needed to address the science objectives. The E2E-iSAG formulated a reference landing site set to (1) demonstrate the ability to find sites that in principle could achieve the highest-priority science objectives and (2) provide environmental conditions to allow engineering planning to take place. The E2E-iSAG evaluated 85 sites previously proposed by the Mars science community. Threshold criteria, based on finding the materials that could address the science objectives and sampling priorities, were applied, and at least 10 sites that address most of the objectives were identified. Of these, seven were selected as "reference sites" because they have a range of properties that would help engineers define landing and roving capabilities and because they already have sufficient imaging to conduct terrain evaluation. In due course, a call for landing site proposals would be made to initiate a comprehensive site selection process similar to those employed for MER and Mars Science Laboratory (MSL).

In response to the proposed ESA-NASA collaboration for a single 2018 joint rover, several assumptions were added to the E2E-iSAG deliberations in May 2011: (1) a single rover delivered by the MSL descent system, (2) the rover would support scientific objectives originating both from MSR and ExoMars in situ planning, and (3) the Pasteur payload would be retained in the rover. The group then evaluated the measurement capabilities in addition to those included in the Pasteur payload that would be required for sample selection and caching, and our conclusions were

(1) Rapidly obtained mast-based mineralogical (i.e., spectroscopic) determinations would be required, in addition to the Pasteur payload macroscopic imaging capability (PanCam) (Required);

(2) Arm-mounted contact measurements include microscopic imaging, elemental chemistry, and mineralogy, preferably with imaging and mineralogy resolved at the submillimeter scale (Required). The capability to detect organic carbon with a contact instrument is also highly desired;

(3) The arm should also include a device capable of removing dust and weathering layers from rock surfaces (Required);

(4) The capability of transferring samples collected by an arm-mounted corer to the Pasteur Analytical Laboratory Drawer (ALD), especially for analyses of organics and mineralogy, and the capability of transferring subsurface samples from the ESA drill to the sample cache are both highly desirable, but the latter capability would have significantly higher priority (Desired).

The E2E-iSAG identified several issues requiring further study as soon as possible. These include questions of science objectives and strategies best addressed by MEPAG, program-level matters directed to both ESA and NASA, and science-engineering research and development requirements, also to be addressed by both NASA and ESA.

In summary, the E2E-iSAG identified science objectives that would drive the scientific analyses conducted on the first samples returned from well-characterized environments on Mars. The group identified the number and types of samples, associated masses and volumes, in situ measurements needed for context, and sample containment requirements needed to meet all or a majority of the stated objectives. The group also identified a reference set of landing sites that could be used to scope the related engineering tasks for addressing successfully the MSR Campaign scientific objectives. The E2E-iSAG anticipates that the results of this unprecedented campaign would fundamentally advance our understanding of Mars, planetary evolution, and the possible origin and distribution of life in the Solar System.

\section{Introduction}

Scientific exploration of Mars has been, and remains, a key component of the space programs of the space-faring nations of the world. Of the planets in our solar system, Mars is the most accessible by spacecraft, the most Earth-like in terms of geological history and environment, and the planet most likely to have hosted an independent origin and evolution of life. The return to Earth of geological and atmospheric samples collected from the martian surface has long been an important goal of planetary exploration, in general, and Mars exploration, in particular. Although planning for such an enterprise has a very long history, the recent successes of various orbital and landed missions to Mars have enhanced the rationale and renewed the impetus to pursue Mars sample return (MSR). As part of this development, in 2009, the space exploration programs of the United States (NASA) and Europe (ESA) began discussion of a formal program of cooperation and collaboration in Mars exploration. In the United States, an important recent development is the recommendation of the 2011 Planetary Science Decadal Survey that a cost-constrained rover mission (with potential launch in 2018), with key in situ scientific objectives, also include a sample cache, and in that sense would become the first element of an MSR campaign. The proposed 2018 rover was judged to be the top priority for NASA-sponsored flagship missions in the coming decade (NRC, 2011).

The Mars Exploration Program Analysis Group (MEPAG), in support of both NASA and ESA, has been actively studying concepts for a collaborative MSR campaign for several years. An important early step was carried out by the MEPAG Next Decade Mars Sample Return Science Analysis Group (MEPAG ND-SAG, 2008) that provided the first extensive evaluation of science priorities and necessary mission capabilities for MSR, within a constrained engineering and budgetary context. As the likely architecture of an MSR campaign came into better focus, the MEPAG Mid-Range Rover Science Analysis Group (MRR-SAG) formulated a mission concept for a 2018 Mars Astrobiology ExplorerCacher (MAX-C). This proposed mission was intended to represent the first element, involving in situ exploration of a compelling site on Mars as well as sample collection and caching, in what could eventually become a three-mission MSR campaign (Fig. 1; MEPAG MRR-SAG, 2010). An additional important study is that of International Mars Architecture for the Return of Samples (iMARS, 2008), sponsored by the International Mars Exploration Working Group.

At about the time of the MAX-C final report (MEPAG MRR-SAG, 2010), the possibility that a mid-range rover and 


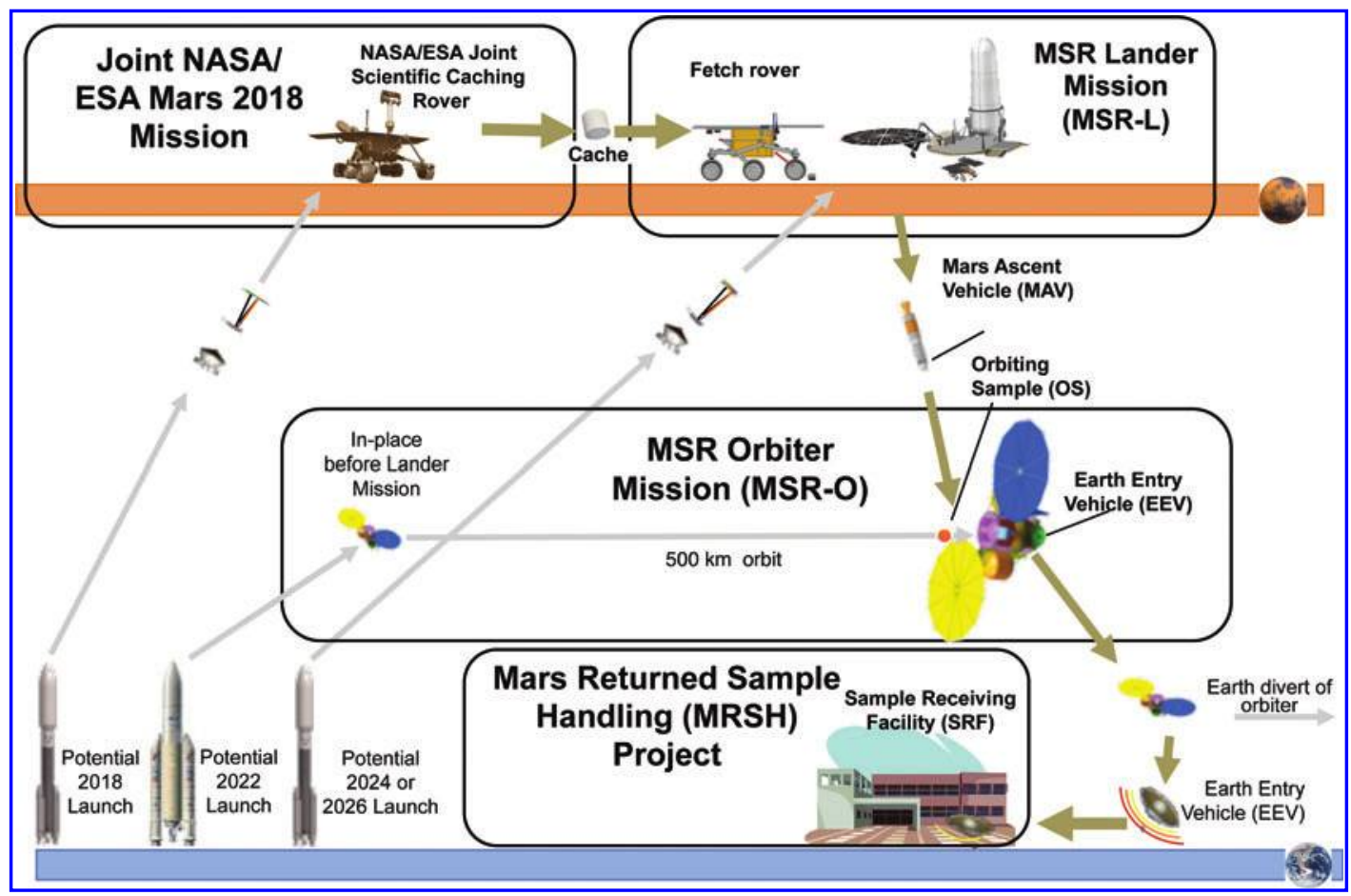

FIG. 1. Mission elements of the proposed NASA/ESA MSR Campaign architecture. Note that the potential 2022 and 2024 (or 2026) launches of MSR-O and MSR-L are nominal only and could take place at later dates. Color images available online at www.liebertonline.com/ast

ESA's ExoMars rover could be delivered to Mars together, and landed at the same site, was proposed. This in turn resulted in an evaluation of the collaborative science that MAX-C and ExoMars could accomplish by the MEPAG 2-Rover International Science Analysis Group (MEPAG 2R-iSAG, 2010). It was within this framework that the deliberations reported here were initiated.

As described in greater detail below, the MEPAG MSR End-to-End International Science Analysis Group (MEPAG E2E-iSAG) was chartered in August 2010 to fully evaluate the implications on the proposed 2018 rover concept of the cache. What would those samples be used for? How many would be needed? How big would they need to be? These, and a myriad of other questions related to the concept of an MSR campaign (Fig. 2), need to be addressed to design the proposed cache and plan for how the samples would be selected and stored. In the midst of our deliberations, technical and cost considerations led to the proposal that the essential functionalities of the MAX-C and ExoMars rovers might be combined into a single joint ESA/NASA rover capable of meeting many, if not most, of the science objectives of both original missions. Although this possibility was
FIG. 2. General approach of the E2E-iSAG deliberations. Color images available online at www .liebertonline.com/ast

\begin{tabular}{|c|c|c|c|c|}
\hline & \multicolumn{3}{|c|}{ Prioritized MSR science objectives } & \\
\hline & \multicolumn{3}{|c|}{ Derived implications } & \\
\hline & \multicolumn{2}{|c|}{$\begin{array}{l}\text { Samples required/desired to } \\
\text { meet objectives }\end{array}$} & $\begin{array}{l}\text { Measurements } \\
\text { on Earth }\end{array}$ & \\
\hline \multicolumn{5}{|c|}{ Critical Science Planning Questions for 2018} \\
\hline $\begin{array}{c}\text { Variations of } \\
\text { interest? } \\
\text { \# of samples? }\end{array}$ & $\begin{array}{l}\text { Types of landing } \\
\text { sites that best } \\
\text { support the } \\
\text { objectives? }\end{array}$ & $\begin{array}{l}\text { Sample } \\
\text { size? }\end{array}$ & $\begin{array}{l}\text { Measurements } \\
\text { needed to interpret } \\
\text { \& document geology } \\
\text { and select samples? }\end{array}$ & $\begin{array}{l}\text { On-Mars } \\
\text { strategies? }\end{array}$ \\
\hline \multicolumn{5}{|c|}{ Engineering implications } \\
\hline $\begin{array}{l}\text { Sampling } \\
\text { hardware }\end{array}$ & $\begin{array}{l}\text { Instruments on } \\
\text { sampling rover }\end{array}$ & $\begin{array}{l}\text { EDL \& m } \\
\text { lifetir }\end{array}$ & $\begin{array}{l}\text { oility parameters, } \\
\text { ops scenario }\end{array}$ & $\begin{array}{c}\text { Sample } \\
\text { preservation }\end{array}$ \\
\hline
\end{tabular}


not part of our initial assumptions, and was considered only late in our deliberations, the E2E-iSAG did attempt to reconcile such a mission concept with its original charter.

\subsection{A proposed MSR implementation architecture}

The currently proposed architecture for the MSR Campaign entails four primary elements: a rover mission to collect and cache the samples; a lander mission (MSR-L) to transfer that cache to orbit; an orbiter mission (MSR-O) to retrieve the orbiting cache and return it to Earth; and a Mars Returned Sample Handling (MRSH) project that would encompass all the functionalities necessary to receive the samples (in a special facility referred to as the Sample Receiving Facility) and provide access to the laboratory infrastructure necessary to carry out the scientific investigation of the samples. Note that the first mission in this sequence (the mid-range rover) would have compelling in situ objectives sufficient to justify it as a standalone mission, and the decision to fly the 2018 rover would not automatically mean commitment to fly the remaining elements of MSR. Also, the length of time the cache would remain at the surface may be indefinite (see NRC, 2011).

The campaign would entail three launches, as depicted in Fig. 1 (Mattingly and May, 2011; NRC, 2011). The current baseline for the first mission is the proposed NASA/ESA Mars 2018 joint rover mission, which would utilize a heritage Mars Science Laboratory (MSL)-style entry, descent, and landing (EDL) system (Steltzner et al., 2006) to land on Mars a jointly developed rover with the capacity to meet both in situ and MSR scientific objectives. This rover would collect and cache samples that would be ready for future pickup. The proposed second mission is the MSR Orbiter (MSR-O), which could be two Mars launch opportunities (i.e., 4 years) later. MSR-O is projected to launch before the MSR Lander (MSR-L) so that it could provide telecommunications infrastructure for the lander mission operations during arrival and after landing. The third mission is the proposed MSR-L, which would also use an MSLstyle EDL system to deliver the lander platform to the surface of Mars. The lander platform would dispatch a fetch rover to retrieve the sample cache. The cache may be augmented by a lander-collected sample of gas and possibly also regolith. The landing platform would also serve as a launch pad for a Mars Ascent Vehicle (MAV), which would be used to insert an orbiting sample container (OS) into a $500 \mathrm{~km}$ near-circular orbit. After monitoring the MAV launch through release of the OS from the MAV, MSR-O would rendezvous with and capture the OS, which would then be packaged into an Earth entry vehicle (EEV) carried with MSR-O. The orbiter would then return to Earth, release the EEV a few hours before entry, and divert into a non-Earth-return trajectory. The EEV would hard land on the surface and then be transferred to a secure sample receiving facility (SRF; see Beaty et al., 2009, and references therein) for quarantine before samples would be made available for scientific research.

\section{MSR Campaign Scientific Aims and Objectives}

\subsection{Introduction}

Several groups, most recently MEPAG (2010) and the National Research Council (NRC, 2011), have outlined the science objectives for Mars exploration. The objectives are diverse enough that no single mission could address them all. It has been recognized for several years that sample return is the single activity that would make the most progress toward the entire list. Most recently, the NRC (2011) concluded that the state of our knowledge of Mars is such that MSR is the next logical major step in Mars exploration and that there are no other strategic missions worth flying before MSR. However, one of the realities of sample-based science is that not all samples are equally useful for all kinds of scientific questions. A first step, therefore, in planning the MSR Campaign is to identify and prioritize the science objectives. This is then input into choosing where to go to get the samples and to determine the many sample-related attributes of the flight hardware such as mission lifetime, EDL capabilities, mobility parameters, sampling capabilities, onMars measurements, and sample preservation capabilities.

The '03/'05 MSR mission was planned in 1999-2000 with seven scientific objectives (listed in Table 1 of MEPAG ND-SAG, 2008), all of which were highly generalized. These objectives provided very little guidance on where to send the mission or the kinds and quantities of samples to collect. Based on the results of the NASA and ESA exploration missions during the period 1996-2007, the MEPAG ND-SAG (2008) proposed 11 much more specific statements of "possible scientific objectives," with the caveat that "the achievable degree of progress towards these scientific questions would depend on the choice of landing site" and several other factors, thereby deferring several essential questions of scientific priority to successor planning teams and to a later landing site selection process.

Several additional recent studies have played a major role in setting the scene for the present update of MSR scientific planning. The NRC's Astrobiology Strategy report (NRC, 2007), MEPAG ND-SAG (2008), iMars Working Group (2008), MEPAG MRR-SAG (2010), and the Decadal Survey report (NRC, 2011) all emphasized that we are now ready to be much more specific about MSR scientific planning, strategies, and priorities, and that astrobiology-related objectives have become important enough to become a driving force behind the mission.

The scientific value of a potential MSR has been discussed in the literature for at least 30 years (see, e.g., NRC, 1978, 1990a, 1990b, 1994, 1996, 2003, 2006, 2007), and the scientific rationale for returning samples has evolved over time. Early studies (e.g., NRC, 1978) emphasized the need for samples to better understand the evolution of Mars as a planet. More recently, however, emphasis has shifted to the search for life (e.g., NASA, 1995; NRC, 2007).

Finally, for the purposes of this study, the vision of the MSR Campaign was constrained by what was considered to be practical (see charter assumptions in Appendix A2). Thus, objectives that might require high latitudes, high elevations, deep ( $>2 \mathrm{~m}$ ) drilling, and large sample masses, for example, were given lower priority. The intent was not to provide a prioritized list in the abstract, but one that could be used to guide a sample return campaign in the context of our current knowledge and expectations of future engineering and fiscal resources.

\subsection{Specific proposed science aims and objectives for Mars returned sample science (organized by topic)}

In the present study, the E2E-iSAG considered all previous statements of scientific objectives for Mars returned sample 
Table 1. Science Objectives Defined by the E2E-iSAG, in Thematic Order

AIM Objective

In rocks interpreted (from orbital and in situ data) to represent one or more paleoenvironments with high potential for past habitability and biosignature

A. Life 1 Critically assess any evidence for past life or its chemical precursors, and place detailed constraints on the past habitability and the potential for preservation of the signs of life.

2 Determine if the surface and near-surface materials contain evidence of extant life.

B. Surface $\quad 1$ Reconstruct the history of surface and near-surface processes involving water.

2 Assess the history and significance of surface modifying processes, including, but not limited to: impact, photochemical, volcanic, and aeolian.

3 Constrain the magnitude, nature, timing, and origin of past planet-wide climate change.

C. Planetary evolution 1 Quantitatively constrain the age, context and processes of accretion, early differentiation, and magmatic and magnetic history of Mars.

2 Constrain the origin and evolution of the martian atmosphere, accounting for its elemental and isotopic composition with all inert species.

D. Human exploration 1 Assess potential environmental hazards to future human exploration.

2 Evaluate potential critical resources for future human explorers.

science and more recent perspectives from a broad segment of the Mars science community, along with its own perspective and judgment. The E2E-iSAG also developed eight implementable statements of scientific objectives, which are organized under four higher-level scientific aims (Table 1). The eight objectives have been prioritized, and it is our recommendation that these priorities guide landing site selection and a broad range of implementation considerations. Whereas the ND-SAG and MRR-SAG objectives encompassed a range of possible MSR missions (for example, a mission to return an ice sample), the present study is intended to be limited to missions that could be implemented with currently plausible resource and political constraints, as reflected in the assumptions presented in our charter (Appendix A2).

2.2.1. Aim A. Life. The question of whether life arose (and possibly still exists) somewhere other than Earth is one of the most fundamental questions asked by humankind, and Mars is a tantalizing target in the quest to find an answer. Of all the bodies in the Solar System, Mars is most similar to Earth and bears evidence of watery, potentially habitable environments in the deep past. Mars and Earth are both generally considered to have been uninhabitable until after the Late Heavy Bombardment ( 4.0 Ga), and on Mars, conditions appear to have become far less hospitable by around 3.5 billion years ago (e.g., NRC, 2007, and references therein). However, on Earth that relatively short length of time was evidently enough to allow life to gain a footholdit is widely accepted that on Earth the fossil record extends back at least as far as almost 3.5 Ga (Allwood et al., 2006, 2009; Wacey et al., 2011). Could life have arisen on Mars during the same time frame? This question is central to Mars exploration, and returning samples from Mars is considered essential for meeting this aim.

There are two recent, and very instructive, case histories of the role of laboratory sample studies in seeking the signs of life: (1) the investigation of the Allan Hills meteorite (ALH84001) and (2) the search for the evidence of life in the oldest rocks on Earth.
- In 1996, McKay et al. presented the hypothesis, based on four lines of evidence, that there is evidence of ancient life in the martian meteorite ALH84001. This triggered a flurry of activity into criteria for distinguishing biotic from abiotic signatures and the way in which these criteria could be applied to this particular rock. Multiple research teams applied every method available in Earth's research infrastructure to the problem. As of this writing, the debate continues (for recent references, see Steele et al., 2007; Niles et al., 2009; Thomas-Keprta et al., 2009; and Treiman and Essene, 2011).

- The studies of putative biosignatures in Earth's Early Archean-age rocks (e.g., Lowe, 1983; Walter, 1983; Schopf and Packer, 1987; Brasier et al., 2002; Allwood et al., 2006, 2009; Schopf, 2006, and references therein) have similarly involved dozens of research teams studying different rock units and different kinds of samples and formulating and testing multiple hypotheses over an extended period of time. We learned from this that evidence of ancient microbial life on Earth is extremely challenging to identify and requires careful integration of diverse lines of evidence across multiple scales, including kilometer- to submillimeter-scale observations in outcrops and centimeter-to submicrometerscale observations in returned samples.

These two case histories illustrate that searching for the signs of life requires intensive multidisciplinary laboratory approaches and the testing and retesting of samples over and over as the hypotheses are progressively refined. Note that a major difference in these two examples, however, is that, in the case of the ancient outcrops on Earth, it was also possible to bring field methods to bear (in addition to laboratory study of samples); this has proved to be essential in providing contextual constraints on the possible biogenic origin of different features. These terrestrial astrobiology studies show that unambiguous interpretations of samples in the laboratory depend on the acquisition of the necessary comprehensive, multiscale geological context in the field, and there are significant implications therein for our strategies on how to approach the search on Mars (see Section 7). 
FINDING \#1: The analysis of samples returned from Mars is considered an essential component of the effort to determine whether life ever arose on that planet.

2.2.1.1. Objective A1: Critically assess any evidence for past life or its chemical precursors, and place detailed constraints on the past habitability and the potential for preservation of the signs of life. Objective A1 has three distinct but strongly interrelated components.

Evidence for life or its chemical precursors. The first component would be to determine whether life or its chemical precursors once existed at the explored location on Mars by analyzing evidence that occurs within the sampled rocks. Importantly, this objective is not to determine whether life ever existed on Mars, as a negative result at one site does not equate to a negative result for the whole planet. Rather, the objective would be to determine whether evidence for life exists in the sampled material, with the expectation that landing sites and samples with a high likelihood of containing such evidence would be targeted.

To ensure that samples with a high likelihood of containing evidence of life would be collected, it is vitally important to narrow the search, as has been shown in studies of early life on Earth, by acquiring a comprehensive understanding of the local geology. What was the past environment? What conditions and processes occurred in the past environment, and how did they change over time and space? How did those variations affect habitability and preservation potential? What are the different deposits and facies that record those environments and processes?

Habitability. The second component of Objective A1 pertains to understanding the habitability of the past environment in which the rocks were formed. For example, if sedimentary rocks are sampled, what was the environment of deposition, and how hospitable was it to life as we know it? Issues of concern include water availability (e.g., water chemistry, longevity of water bodies), availability of energy sources (e.g., sunlight, chemical gradients), and availability of organic carbon.

A significant body of literature exists on the potential habitability of different martian environments through time (e.g., Boston et al., 1992; Jakosky et al., 2003; Nisbet et al., 2007; Squyres et al., 2008; Tosca et al., 2008; Smith et al., 2009; Fairén et al., 2010; Johnson and Pratt, 2010). In particular, significant advances in the understanding of martian habitability through time have been made as a result of recent missions, including Phoenix and the Mars Exploration Rovers (MERs), and would continue to be made as future missions such as MSL continue to provide new insights at different locations. Two significant insights arising from recent in situ exploration efforts are (1) the existence of liquid water at the surface of Mars in the past (e.g., Squyres et al., 2004a, 2004b; Grotzinger et al., 2005) and (2) the heterogeneity of martian conditions through time and space. Because of this heterogeneity, habitability assessments are not something that could be done once for the whole planet (as is true of some other kinds of measurements). Rather, it would need to be done at every site where evidence of life is sought.

Determining paleohabitability at any given site has multiple purposes. One purpose would be to guide the exploration process toward the locations with best promise and to understand which specific materials within a deposit are more likely to contain evidence of life. Another purpose would be to help understand possible reasons for the absence of biosignatures if none are found. If the geological record indicates sustained habitable conditions and ideal processes for biosignature preservation, then the absence of biosignatures may truly reflect the absence of a martian biosphere. However, if the local geological setting indicates habitable conditions were short-lived or processes were not ideal for biosignature preservation, then the absence of biosignatures would have more limited bearing on the presence or absence of a martian biosphere.

Finally, it is worth noting that biosignatures can be transported from a more habitable location to a less habitable one. For example, the toe of a lacustrine delta that had biological organic matter within it has as much (or more) to do with the habitability of the hinterland than the habitability of the lake. Thus, biosignatures may occur at the less habitable location because of the existence of mechanisms to transport, concentrate, and preserve the biosignatures there.

Preservation potential. The third component of Objective A1 relates to understanding the potential for signs of life or abiotic organic matter to be preserved in the rocks. "Preservation potential" simply refers to the notion that in order for evidence of life to be detectable, it must have survived all the geological processes that affected the rocks. Numerous geological processes, including erosion, oxidation, recrystallization, physical deformation, and chemical alteration, can erase the signs of life. Characterization of the environmental features and processes that preserve specific lines of evidence for life is a critical prerequisite in the search for life (MEPAG, 2010).

Different types of biosignatures require different conditions for preservation: these are discussed in the MEPAG Goals document and in the scientific literature (e.g., Summons et al., 2011, and references therein). As with habitability, preservation potential serves to target materials that are more likely to contain evidence of life and to help understand possible reasons for the absence of biosignatures if none are found.

An important additional benefit of investigating habitability and preservation potential is that the palaeoenvironmental and broader geological interpretations done to determine habitability and preservation potential would also provide essential contextual framework for the recognition (detection, interpretation) of any biosignatures that may exist.

FINDING \#2: Credible strategies to search for evidence of past life in the geological record are fundamentally dependent upon simultaneously evaluating past habitability potential and determining the potential for preservation of ancient biosignatures.

Past or present life? Given our current state of knowledge of Mars, we have very clear strategies for how to explore for past life on Mars (see e.g., NRC, 2007, 2011; MEPAG, 2010). There are criteria for prioritizing landing sites and, once on the ground, strategies for what to sample (see later sections of this report). By contrast, it is much less clear that indigenous martian life could exist today at, or close to, the martian surface where it could be accessed by our present exploration systems. The general surface environment, in which the rover would operate, with its low water activity, high UV radiation, and low temperatures, would be very hostile. 
Moreover, if life could exist on Mars today, it is not clear where we would be most likely to find it. Hypotheses exist for possible present-day martian near-surface habitats; but until more is known, setting an objective to locate and return a sample likely to contain a live martian organism would be akin to searching for a needle in a haystack, that is, relying on a strategy of hope. Thus, as of this writing, we do not have a means of using extant life search parameters to prioritize landing sites or to prioritize samples at the site. It is of course entirely possible that during the landing site selection process for the MSR Campaign somebody would be able to make such arguments, and if so, they need to be considered. For now, however, our judgment is that we should concentrate our strategic planning on the search for ancient life.

The current assumption is that all returned samples would have the potential for the presence of extant life. However, since we do not have a way of assessing this potential during rover operations, or using it in scientific sample selection, we have to assume that this potential exists equally for all returned samples (that were selected for other reasons). Therefore, we would want to do extant life testing on all samples, dealing with them as if they come to us "blind." (This is the reason the extant life objective cannot easily be cross-prioritized with the others).

FINDING \#3: Analysis of the returned samples for extant life would be a high-priority science objective, but we have not found a logical way to effectively incorporate this into landing site and sample selection on Mars.

Candidate types of evidence for past life on Mars. Any evidence of past life on Mars is assumed to consist only of relicts of primitive microorganisms, as opposed to the types of more "advanced" organisms that required billions of years to evolve on Earth. This is based on the assumption that the surface of Mars was habitable only for a relatively short time, and thereby only a short window of opportunity for life to evolve occurred (and, of course, we do not know if life took advantage of this opportunity). The phrase "chemical precursors to life" is used here to refer to organic materials that may have been involved in processes that could be considered precursors to biological processes.

A biosignature is a signal or feature of biological origin. There are many different kinds of biosignatures, and they can be grouped into four major categories:

(1) Macro-morphological: stromatolites and other microbialites, reefs and bioherms, microbial textures and fossil microbial mats, and microbially induced structures

(2) Micro-morphological: microfossils, endolithic microborings

(3) Chemical fossils: such as biogenic carbon isotopic fractionation patterns or biologically derived trace element distributions

(4) Molecular fossils, or "biomarkers" (organic molecules of biological origin)

Although organic matter is sometimes referred to as a biosignature, organic material is not, of itself, a biosignature, as it is possible to have organic matter from nonbiological sources. Evidence of the biogenicity of organic matter may come in the form of biomarkers, chemical fossils or microfossils, or association with a macro-morphological fossil.
The role of field work to achieve Aim A. Field observations are fundamental to the interpretation of potentially biogenic features. Ambiguity about potential evidence of life in martian meteorite ALH84001 (e.g., Golden et al., 2001) highlights the challenges of interpreting microbial signatures on the basis of evidence contained in limited quantities of material without larger geological context. To interpret biosignatures-and in some cases even to detect them at all-it is essential to understand the local geological setting in adequate detail and across multiple scales. The extent and type of contextual detail required can depend on the particular biosignatures and geological setting in question, so it is essential to preemptively acquire as much contextual information as possible while "in the field."

FINDING \#4: Accurately interpreting potential biogenic features in the geological record is dependent upon a detailed understanding of geological context of those features, acquired through careful in situ field observations at multiple scales across the area in which the signatures occur.

\section{Sample types of interest}

\section{Primary target types:}

1. Subaqueous sediments. The term subaqueous sediments refers to all water-deposited surface sediments whether deposited by standing or flowing surface waters, or by discharging or seeping subsurface waters (e.g., playa sedimentation, tufa style deposition, travertines). Such deposits include both clastic and chemical deposits.

Of particular interest for sampling are (for example): sediments that are potentially biologically precipitated (e.g., carbonate), sediments that may contain concentrated organic materials (e.g., black shale), sediments that have high potential for preserving microfossils (e.g., chert), or sediments associated with potentially biogenic morphological features. In addition, any rocks that contain high degrees of primary textural preservation would be valuable, and any materials containing well-preserved sedimentary structures, textures, and fabrics (such as lamination) would be of interest both to observe and measure in situ and to sample, because these provide essential insights for paleoenvironmental interpretation. Large crystal facies such as selenitic gypsum beds in evaporites would be of interest for their potential to preserve microfossils and other biosignatures (e.g., Rouchy and Monty, 1981) as well as fluid inclusions and geochemical proxies for palaeoenvironmental conditions.

In a broader sense, it is essential to target rock formations in which the relative positions (vertical or horizontal) of different layered deposits could be mapped. These relationships allow interpretation of stratigraphic age, paleoenvironment, and mineralogical or sedimentological gradients (equivalent to reading the pages of a book in the right order). The greater the degree of lateral or vertical correlation, or both, the better we would be able to develop context, make informed sample selection, and interpret future sample analyses.

2. Hydrothermal sediments. Hydrothermal depositssediments refer to geological materials deposited at the surface from warm circulating fluids derived from igneous or impact-driven activity. The sediments may originate in subaqueous or subaerial form (e.g., from thin sheets of flowing 
water or spray). Environments of interest include sinter (which is subaerial) or subaqueous sediments like those surrounding submarine hydrothermal vents. Significant amounts of research over the past several decades have shown that hydrothermal sedimentary environments are not only widely inhabited on Earth by extremophilic organisms but are also excellent locations for preserving biosignatures due to the rapid mineralization that occurs as supersaturated waters are exhaled from the subsurface (e.g., Walter and Des Marais, 1993; Cady and Farmer, 1996; Des Marais, 1996; Walter, 1996; Farmer and Des Marais, 1999; Farmer, 2000).

High-priority targets for sampling include precipitated sediments with high degrees of primary textural preservation (particularly fine lamination); any sediments with local textural, structural, or compositional variations that could be biogenic; and primary precipitates that may preserve fluid inclusions, organic matter, or geochemical clues to past conditions. Suites of samples representing variation across chemical and physical gradients in hydrothermal environments-such as proximal-distal sample sets around a hydrothermal vent or textural preservation gradients-would be especially valuable for developing context and interpreting variations in habitability and preservation potential. As with subaqueous sediments, rock formations in which the relative positions (vertical or horizontal) of different layered deposits could be mapped would be the most valuable.

Secondary target types:

3. Rocks altered by hydrothermal fluids. Hydrothermally altered rocks refer to formations of any origin altered by fluids that originate from magmatic or volcanic activity and, hence, encompass typically higher-temperature fluids. The extent of high-temperature fluid-rock alteration may reduce the information available about past life due to deformation and alteration of the chemical, mineralogical, textural, and stratigraphic state of the original deposits. The existence of a subsurface biosphere on Earth (Onstott et al., 2006; Chivian et al., 2008) illustrates the potential for life to exist in similar subsurface environments on Mars, namely, subterranean interstices where liquid water is (or was) available. However, while the potential for habitability clearly exists, little is currently known about the taphonomic potential (potential for preservation of biosignatures) in subsurface settings characterized by fluid-driven rock alteration. Alternatively, processes such as silicification may enhance preservation potential (Westall et al., 2006; Orange et al., 2009).

4. Rocks altered by low-temperature (meteoric) fluids. In contrast to hydrothermal alteration, "low-temperature fluidaltered rocks" include rocks altered by interaction with subsurface meteoric fluids, or what are loosely called formation fluids. Typically, such fluids have a lower-temperature origin compared to hydrothermal (magmatic or volcanic) fluids.

Desired samples would include carbonate, chert or other fracture-fill minerals precipitated from waters moving through subsurface fractures, and altered ultramafic lithologies. Possible terrestrial analogues for the latter include low-temperature serpentinization systems such as those that occur today in peridotite-hosted environments, and radiolytic decomposition of water in deep crystalline rocks of the Witwatersrand Basin that have been shown to support $\mathrm{H}_{2}$ autotrophs in the absence of any modern-day volcanic/magmatic activity (Lin et al., 2006; Sherwood Lollar et al., 2006).
Fluid-rock alteration, even at low temperatures, may reduce the information available about past life due to deformation and alteration of the chemical, mineralogical, textural, and stratigraphic state of the original deposits. However, evidence (for instance) of serpentinization provides evidence at least of habitability - a potential geochemical basis for chemolithotrophic life (generation of $\mathrm{H}_{2}$ and associated hydrocarbon-producing reactions) (Chapelle et al., 2002; Lin et al., 2006; Sherwood Lollar et al., 2006).

\subsubsection{Aim B. Surface}

2.2.2.1. Objective B1. Reconstruct the history of surface and near-surface processes involving water. Knowledge of the past history of water is essential for understanding past habitability and climates and for understanding the sequence and nature of the geological processes that have affected the surface. The aqueous history of Mars can be divided into three eras: (1) the Noachian, for which we have evidence that suggests widespread episodic precipitation, fluvial erosion, lacustrine sedimentation, weathering, groundwater activity, and possibly oceans; (2) the post-Noachian, characterized dominantly by low erosion rates and cold, dry conditions punctuated by episodic floods widely spaced in time; and (3) the recent geological past, for which the water story is dominated by gully formation, glacial activity, thin aqueous alteration rinds on exposed surfaces, and changes at the poles (Carr, 1996; Carr and Head, 2010). This last period includes modern Mars, where observations of seasonally recurring transient dark streaks extending downslope point to the possible local flow of briny liquid at the surface of Mars today (McEwen et al., 2011). The period of most interest for sample return is the Noachian, for which we have the best evidence of sustained, widespread presence of liquid water at the surface (Carr, 1996). On the other hand, younger hydrothermal deposits, if detected and accessible to sampling, would also be of considerable interest.

\subsection{Sample types of interest-B1}

1. Sediments from long-lived lakes. Included here are Noachian or early Hesperian delta deposits and other layered deposits whose composition and location (e.g., in a low area with convergent drainage) suggest deposition in a standing body of water (Malin and Edgett, 2003; Cabrol and Grin, 2010; Grotzinger et al., 2011). Locations are identified from geomorphic context, depositional structures, and the presence of evaporites (Grotzinger et al., 2005). Samples from such environments should include both clastic and chemical sediments. Sample sizes should be large enough to preserve sedimentary structures and must be acquired and packaged to preserve such structures. In-place sampling is strongly preferred over float. A suite of samples from a vertical section is desirable to assess how the depositional conditions changed with time and what post-depositional changes have occurred. Samples should be located so that their stratigraphic age, if not their absolute age, could be narrowly constrained.

2. Hydrothermal deposits. As discussed above in connection with Objective A1, hydrothermal deposits are of interest not only for their relevance to the history of water action but also because of their potential habitability and their potential for preserving organic remains (Farmer, 1998). Deposits are identified in volcanically active regions of the past or present 
(or, alternatively, those regions affected by large impact events) from the presence of primary or secondary aqueous minerals and indications of strong chemical fractionations. Individual samples need not be large, but multiple samples are highly desirable because of the potential for strong chemical and physical gradients in hydrothermal environments. Stratigraphic age is of secondary importance.

3. Fluvial deposits. Fluvial deposits are of interest for the history of water action because the sedimentary structures indicate the nature of the fluvial regimes that cut the channels and valleys. There are two broad types of fluvial features: (1) branching valleys mainly in the Noachian and (2) large floods mainly in the Hesperian. The branching valleys would be the main interest for sample return. Samples of sediments deposited by the streams that cut the valleys have the potential for providing clues as to whether the streams were persistent or episodic, what their discharges were, what the climatic conditions were when they formed, and what the timescale was over which the valleys were cut (Carr, 1996; Howard, 2007). A preferred sampling site would be a Noachian flood plain as indicated by a flat-floored valley with a sinuous channel. Other lower-priority possibilities are alluvial terraces and alluvial fans. Samples should include both clastic and chemical sediments, if present. Samples should be large enough to preserve sedimentary structures and acquired and stored so as to preserve such structures. Sampling should be in place, and a suite of samples from different stratigraphic positions is desired so that changes in fluvial regimes with time could be assessed. Boundaries between sets of fluvial deposits would be of interest for assessing conditions in the intervals between fluvial episodes.

4. Low-temperature alteration products. Widespread presence of aqueous minerals and valley networks in Noachian terranes suggests at least episodic warm, wet conditions at that time. A major issue is whether the warm conditions were short-lived transient events such as might be caused by large impacts (Segura et al., 2002), or whether the conditions were long lasting with precipitation and runoff in quasiequilibrium with evaporation from large bodies of water (Baker, 2001). Sampling of ancient, lithified soil profiles may reveal which model is most likely. The locations to be sampled would be identified from the mineralogy as revealed by orbital observations. The main interest here is chemical and mineralogical changes up and down the soil profile. Samples would not need to be large, but multiple samples are strongly desired.

Many of the sample types of interest for this objective may be poorly indurated and susceptible to breakup during coring. This may make preservation of sedimentary structures and layering on the millimeter to centimeter scale difficult. Efforts should be made to design the sampling system so as to minimize disintegration of the cores for poorly indurated samples.

2.2.2.2. Objective B2. Assess the history and significance of surface-modifying processes, including, but not limited to, impact, photochemical, volcanic, and aeolian. Much of the geological history of the martian surface is recorded in rocks, sediments, and soils whose compositions are not dominated by surface and near-surface aqueous processes but nevertheless provide fundamental insight into many of the high- est-priority research investigations defined by MEPAG. Included in these processes are impacts (Melosh, 1989), atmospheric/photochemical effects (Levine, 1985; Quinn et al., 2006; Yen et al., 2006), volcanism (Wilson and Head, 1994), aeolian transport/deposition (Sullivan et al., 2005), and evaluation of the fate of carbon in near-surface environments (Kminek and Bada, 2006).

\subsection{Sample types of interest-B2}

1. Volcanic unit with known stratigraphic age. Although martian meteorites provide a sampling of volcanic and plutonic crustal rocks, the lack of geological context and their restricted, largely nonrepresentative, age ranges limit their value for understanding the planet's volcanic evolution (see Section 2.2.3). Accordingly, a primary purpose of obtaining unaltered volcanic rock samples would be to obtain absolute radiometric ages (see MEPAG E2E-iSAG, 2011, for a summary of geochronology approaches). Obtaining such ages from a post-Noachian volcanic unit (e.g., middle Hesperian) with known stratigraphic age or crater density would provide critical calibration to the martian stratigraphic timescale (Hartmann and Neukum, 2001). Such a calibration point would also greatly improve our ability to quantitatively date the martian surface with crater-counting techniques. To relate the volcanic age to the cratered surface confidently, it would be crucial to obtain in-place samples.

In addition, geochemical, mineralogical, textural, and isotopic data for a well-dated volcanic rock of known geological context would also provide important constraints on its magmatic history and on the geochemical nature and evolution of its mantle sources, relevant to Objective C1. This is discussed below under Section 2.2.3. Finally, although unaltered samples are important to obtain the best radiometric dates, any surface alteration rind that might exist on a volcanic rock sample would record interactions between the rock surface and the martian atmosphere, including the UV environment.

A suite of volcanic samples is highly desired but not necessary to achieve the major goals. Experience with martian meteorites demonstrates that it is possible to obtain precise radiometric dates by using mineral isochrons from individual rocks. On the other hand, geochemical and isotopic variation among petrogenetically related whole rocks would provide greatly improved constraints on the origin and magmatic history and thus provide important context to any volcanic samples that might be dated.

2. Impact breccias from large Noachian crater or basin. Impacts have had a profound effect in sculpting the martian surface and redistributing and altering the surface materials, particularly early in the planet's history (e.g., Grant et al., 2008; Rogers, 2010; Barnhart and Nimmo, 2011; Marinova et al., 2011). Impacts may also have had significant effects on climate (Segura et al., 2002). Experience from lunar studies demonstrates the value of impact breccias to help better understand impact processes, to better characterize the lithological diversity of a planetary body, and to better interpret the geological history of a planet's crust. Breccias derived from large craters or basins, which occur mainly in the Noachian, would be particularly important because they sample large areas to considerable depths. They therefore have the potential for acquiring a far more diverse sample than is represented by the primary, in-place rocks at a chosen 
landing site. Because the Noachian era is also the time for which we have the best evidence for aqueous processes (e.g., Hynek and Phillips, 2003; Howard et al., 2005; Poulet et al., 2005; Bibring et al., 2006; Mustard et al., 2008; Murchie et al., 2009), the breccias may contain evidence for aqueous alteration during the impact event, and in addition individual clasts would contain evidence of aqueous conditions prior to the impact event. Breccias formed from a large Noachian impact are also likely to provide a sampling of the deep martian ancient primary crust (for which samples are not available in the current meteorite collection) and possibly even the upper mantle. If the impact breccia is sampled in place, this would add considerable confidence to our ability of relating the breccia to a specific impact event. Nevertheless, a well-chosen float sample would still be of considerable interest.

A suite of impact breccia samples would be highly desired but not necessary. A single sample core could be relatively small yet still contain a number of breccia clasts, depending on clast size. Even a single carefully selected impact breccia sample from a large Noachian impact basin would greatly improve our understanding of lithological diversity within the ancient martian crust. However, multiple samples of impact breccia would more likely provide greater lithological diversity. In addition, multiple samples would mitigate the science risk that only a small number of fragments (or even a single large fragment) are immediately beneath the surface and dominate the sample.

3. Regolith. For the purpose of this report, we use the definition of regolith as the entire layer of fragmental and loose, incoherent, or unconsolidated rock material of any origin that mantles more coherent bedrock (Gary et al., 1972). It includes soil, defined as any loose, unconsolidated material that can be distinguished from bedrock or strongly cohesive sediments but has no singular origin; airfall dust, which is fine-grained material that has settled from the atmosphere; and aeolian deposits, which represent any accumulation of windblown sediment that occurs in recognizable bedforms or as sand sheets.

The origin of the soil component of the regolith of Mars is complex (Banin, 2005), having been influenced by a variety of both nonaqueous and aqueous processes (e.g., Yen et al., 2005; Sullivan et al., 2008; McSween et al., 2010). Although the composition of measured dark-toned soils is broadly similar at all the landing sites visited to date (e.g., Yen et al., 2005), there are also strong, and in places even dominating, local influences on composition due both to the presence of exotic local rock compositions and to the occurrence of secondary alteration, including hydrothermal processes (e.g., Wang et al., 2008; Yen et al., 2008). Soils also interact with the atmosphere, and accordingly their composition is likely to be influenced by the intense UV photochemical environment at the surface (e.g., Yen et al., 2000; Hecht et al., 2009). Understanding photochemical processes, for example, could be crucial in understanding the aqueous versus nonaqueous redox environment of the near surface and in evaluating the fate of carbon in the near-surface environment (Kminek and Bada, 2006). Another feature of soil samples is that they are likely to contain a diverse suite of rock fragments (e.g., Sullivan et al., 2008), and the study of such fragments would greatly increase our understanding of the geological diversity of the martian crust.
A suite of regolith samples that includes the different components in isolation is highly desired but not necessary to achieve the scientific goals discussed in the preceding paragraph (goals related to human explorations are different, as presented below). Multiple samples would better represent the range of diversity, but great advances on this objective could be achieved with even a single sample of generic soil. The decision on how many soil samples to take needs to be made by the science team of the future, depending on what would be available to them at the landing site.

Although there could be some advantage in being able to do a crude separation of grain sizes in a soil sample with either rakes or sieves to maximize the number of fragments, such a process could also adversely affect the evaluation of atmosphere-regolith interactions, and accordingly, sampling bulk soil is deemed sufficient (see MEPAG E2E-iSAG, 2011).

4. Aeolian sediments and sedimentary rocks. Next to impacts, the physical surface of Mars is most dramatically influenced by the action of wind. Samples of both relatively recent and ancient wind-blown sediment would provide important constraints on aeolian processes over martian geological time that could not be obtained from remote sensing or in situ observations. For example, detailed grain size analyses, examination of grain surface textures, and lithological diversity of grains could be studied. In addition, aeolian sediments can have widespread provenance, and examination by modern sedimentary petrology and detrital geochronology techniques on individual grains (e.g., Hemming, 2004) offer the possibility of greatly expanding our understanding of geological diversity and of providing statistically based age distributions for the martian surface (see MEPAG E2E-iSAG, 2011, for further discussion). For ancient aeolian sedimentary rocks there would also be advantage in obtaining in-place samples to ensure the sedimentological context (e.g., ensure the depositional setting is indeed aeolian), but a carefully selected sample of loose rock would suffice.

A suite of aeolian sediments is highly desired but not necessary. As with regolith samples, widely spaced aeolian deposits would likely provide increased understanding of the diversity of provenance and sedimentary processes. On the other hand, even single samples would likely reflect multiple sources and so would be useful. Accordingly, any lithified sedimentary rock confidently identified as having been deposited in an aeolian environment, either by remote sensing or a previous surface exploration, would be sufficient for an ancient aeolian sedimentary rock sample. Relatively recent aeolian deposits are so ubiquitous that they would likely be available in any site that could be visited for sample return.

To achieve Objective B2, it would be necessary to obtain samples that have interacted with the surface (e.g., photochemical effects) as well as to obtain pristine samples (e.g., datable volcanic rocks). Accordingly, the sampling system should have the capability to sample altered rock surfaces and, concurrently, obtain sufficient mass of fresh sample beneath the surface. This latter requirement could be achieved either by having a rock abrasion tool capable of removing altered surfaces and/or a sampling system that would obtain cores that are long enough to confidently sample the subsurface. From MER experience, the altered 
surfaces are mostly on the order of a few millimeters thick but quite variable and, depending on the specific geological history of aqueous activity, could be anywhere in the range of $<1 \mathrm{~mm}$ to perhaps as much as $\sim 10 \mathrm{~mm}$ thick (e.g., Haskin et al., 2005; Hurowitz et al., 2006; Knoll et al., 2008).

2.2.2.3. Objective B3. Constrain the magnitude, nature, timing, and origin of past planet-wide climate change. Mars experienced extreme changes in environmental and climatic conditions throughout its geological history (Bibring et al., 2006; Mustard et al., 2008). The changes vary greatly in magnitude and timing. The most extreme changes occurred across the Noachian-Hesperian boundary. At this time, surface conditions appear to have changed dramatically. While the Noachian retains a well-preserved sedimentary record of fluvial and lacustrine activity (e.g., Pondrelli et al., 2008; Grant et al., 2010), such records are much less common in younger deposits. Erosion rates also declined precipitously, production of hydrated minerals such as phyllosilicates declined, and deposition of sulfates became more common (Bibring et al., 2006). All these observations are consistent with a change from Noachian time when liquid water was at least episodically stable at the surface and the planet had an active hydrologic cycle to a time when conditions were such that liquid water was only rarely available at the surface. While the observational evidence of warm, wet conditions in the Noachian followed by a change to cold, dry conditions by the late Hesperian is compelling, what caused the hospitable conditions in the Noachian and how persistent they were are unknown. Were conditions during the Noachian such that times favoring fluvial/lacustrine activity were short and episodic, being separated by long dry periods, such as might result from large impacts (Segura et al., 2002); or were the warm, wet conditions semipermanent? Rhythmic sedimentation is a common characteristic of Noachian lacustrine sediments (Lewis et al., 2008a). Were there rhythmic climate changes during the Noachian such as might be caused by periodic changes in obliquity?

The causes of the warm, wet conditions on early Mars remain one of the planet's most puzzling issues. Early core formation and massive loss of hydrogen probably left both the mantle and atmosphere oxidized (Kuhn and Atreya, 1979; Stevenson et al., 1983; Pepin, 1994) and dominated by $\mathrm{CO}_{2}$ and $\mathrm{H}_{2} \mathrm{O}$. However, due to the low early solar luminosity and cloud formation, conditions were such that the surface would not have warmed to temperatures higher than about $230 \mathrm{~K}$, given a $\mathrm{CO}_{2} / \mathrm{H}_{2} \mathrm{O}$ atmosphere, regardless of how thick it may have been (Kasting, 1991). In addition, with Mars' low gravity, a thick atmosphere likely would not have endured against blow-off by large impacts (Melosh and Vickery, 1989), and a warm $\mathrm{CO}_{2} / \mathrm{H}_{2} \mathrm{O}$ atmosphere tends to self-destruct by forming carbonates. Carbonates are detected at the martian surface (e.g., Bandfield et al., 2003; Ehlmann et al., 2008b), although not in the amounts expected from a massive $\mathrm{CO}_{2} / \mathrm{H}_{2} \mathrm{O}$ atmosphere. Sampling of gases trapped in samples that date from this early warm era would provide vital clues on the nature of the early atmosphere and how it may have evolved.

Small climate changes probably occurred throughout the planet's history after the major Noachian-Hesperian transition as a result of obliquity changes (Laskar et al., 2002). These changes are, however, most evident at high latitudes, and understanding these changes would not be a major goal of early sample return except insofar as they have left evidence in sedimentary sequences at low latitudes.

\subsection{Sample types of interest-B3}

Clues as to the climatic conditions during the Noachian and through to the late Hesperian, by which time conditions were probably similar to the present, could come from various sources. While significant information is expected to come from future in situ missions such as MSL and Mars Atmosphere and Volatile Evolution Mission (MAVEN) (Jakosky and Millour, 2011), the return of samples would provide direct access to critical evidence about past climate. One source of evidence would be the chemistry of the present-day atmosphere, particularly the noble gases, as discussed in Section 2.2.3.2. Another significant source is the isotopic composition of volatiles, particularly $\mathrm{H}$ and $\mathrm{O}$ trapped in minerals of different ages, either chemically bound or in vesicles. How the isotopic compositions change with time would provide valuable time-tagged clues on losses from the upper atmosphere that would complement the present-day noble gas data. One issue, for example, concerns the $\mathrm{D} / \mathrm{H}$ ratio, which is unknown for the Noachian. Is the current high $\mathrm{D} / \mathrm{H}$ the result of events early in the planet's history or the result of long-term losses throughout the history of the planet? A fourth source would be the morphological evidence from climate-sensitive geological processes such as fluvial and lacustrine sedimentation. Sedimentary structures such as layering, grain-size distribution, rounding, sorting, and so on, would indicate the conditions under which deposition occurred and how the conditions varied with time. A fifth source is from soils. We know that weathered minerals are common in Noachian terranes and absent in younger terranes (Bibring et al., 2006; Murchie et al., 2009). If soil profiles are present between superimposed deposits such as lava flows, they would provide valuable clues as to the then-prevailing climatic conditions. Accordingly the following samples are desired:

(1) A suite of sedimentary rocks, both clastic and chemical, of different ages that span the Noachian-Hesperian boundary. The suite should include both landing-site scale geological units and outcrop scale differences in order to assess vertical and lateral facies variations. The intent would be to assess the conditions under which erosion and sedimentation occurred and to see how sedimentary environments changed with time across the boundary. Samples acquired from outcrop would be preferred over float samples. The sample must be large enough-and collected and stored carefully enough - such that sedimentary structures would be preserved.

(2) Samples of a pedogenic profile and/or a weathering profile to assess the conditions under which the soil development and weathering occurred. Samples including the different alteration levels/stages should be collected, ranging from the pristine rock through to the stage of maximum alteration. In-place sampling would be required.

(3) Weathered rocks of different ages to determine the isotopic composition of volatile species at different times 
To collect the best possible suites, the sampling system should be able to

- acquire and keep different samples isolated from each other;

- collect fresh samples below recent weathering rinds;

- preserve, within each sample, the stratigraphic orientation (up/down), the stratification, and depositional structures.

The terrane identification and selection for the sedimentary suite would be relatively straightforward in terms of surface age (Noachian-Hesperian boundary), but it has to include sedimentary sequence(s) crossing that boundary in a clear stratigraphic relationship with datable volcanic or other units, which in turn would allow for a reliable age determination from remote sensing. The pedogenic/weathering profile suite would be linked to a Noachian terrane with a range of both secondary and primary minerals, detected and characterized by remote sensing and further constrained and analyzed by in situ experiments prior to acquisition and caching. For weathered rocks of different ages, only small amounts would be needed. The most important factor with respect to these samples would be that their surface age must be known from remote sensing. The weathered samples should not be from float.

\subsubsection{Aim C. Planetary evolution}

2.2.3.1. Objective C1. Quantitatively constrain the age, context, and processes of accretion, early differentiation, and magmatic and magnetic history of Mars. Understanding the main phases of planetary-scale geological evolution of Mars is essential to providing context for other scientific objectives. Significant insights into planetary-scale evolution of Mars have been derived from studies of martian meteorites-the only samples available for research until MSR occurs. Such studies provide a complementary approach to Mars exploration (McCoy et al., 2011), but the meteorite studies have certain limits. The martian meteorites are all igneous rocks, formed from the eruption or emplacement of mafic magmas through variable degrees of accumulation of olivine or pyroxene (e.g., McSween, 1994) that were ejected from Mars through impact within the past few million years (Nyquist et al., 2001; Fritz et al., 2005). Their martian origin was established through the discovery of high ratios of ${ }^{40} \mathrm{Ar} /{ }^{36} \mathrm{Ar}$ and ${ }^{129} \mathrm{Xe} /{ }^{132} \mathrm{Xe}$ in trapped gases in shock-altered phases of the Antarctic meteorite Elephant Moraine A79001 that closely resembled the martian atmosphere measured by the Viking lander (Bogard and Johnson, 1983). The majority of the 55 known martian meteorites date from the younger Hesperian to the Amazonian (1.3 Ga and 200-600 Ma; Nyquist et al., 2001); ALH 84001 is the only sample from the Noachian (4.09 Ga; Lapen et al., 2010). Several attempts have been made to identify the source craters of the martian meteorites based on spectroscopy of laboratory samples compared to spectra from Mars Global Surveyor Thermal Emission Spectrometer (MGS-TES), with only limited results (Hamilton et al., 2003; Lang et al., 2009). Thus, the martian meteorites lack "field" context and cannot be used to provide ground truth for orbital missions.

Detailed studies of the mineralogy, petrology, and geochemistry of the martian meteorites have yielded insights into the geological evolution of Mars, including the timing of differentiation, mantle mineralogy and water content, and the thermal state of the interior (e.g., Stolper and McSween 1979; Bertka and Fei, 1997; Borg et al., 2003; Leshin and Vicenzi, 2006; Papike et al., 2009). Initial ${ }^{87} \mathrm{Rb} /{ }^{86} \mathrm{Sr}$ and ${ }^{87} \mathrm{Sr} /{ }^{86} \mathrm{Sr}$ values for meteorite whole rock samples plot on a line with a slope corresponding to $4.5 \mathrm{Ga}$ (Borg et al., 1997), and a similar $4.5 \mathrm{Ga}$ isochron emerges from $\mathrm{Sm}-\mathrm{Nd}$ isotopic compositions (Borg et al., 2003). The $4.5 \mathrm{Ga}$ "age" is interpreted to represent the signature of ancient mantle sources established early in the differentiation of the martian interior via crystallization of a magma ocean (Borg et al., 2003). In this scenario, isotopically distinct mantle reservoirs remain unmixed and undisturbed until the melting event that produces a magma, which crystallizes in a near-surface intrusion or lava flow (at 200-600 Ma, in the case of the basaltic martian meteorites). Alternatively, some workers have suggested that the same meteorites have ancient crystallization ages (4.0$4.5 \mathrm{Ga}$ ) and that younger ages reflect the timing of resetting by impact or metasomatism (e.g., Bouvier et al., 2008). Much of the debate about crystallization ages of martian meteorites stems from the lack of geological context, as well as the overprinting of original geochemical signatures by the effects of impact shock (e.g., Fritz et al., 2005). If the 200-600 Ma ages are indeed crystallization ages, as most workers contend, the overrepresentation of Amazonian rocks is attributable to a sampling bias toward younger, more competent igneous rocks; older rocks representing primitive martian crust and weakened by impacts and aqueous alteration are discriminated against in the launch process (Walton et al., 2008, and references therein).

Recent results of Mars surface exploration demonstrate that the martian meteorites are not representative of the bulk of the rocks found at the martian surface (McSween et al., 2009), which raises doubt as to whether the geological evolution of Mars inferred from the meteorites can be applied to Mars as a whole. As an example, basaltic martian meteorites have $\mathrm{CaO} / \mathrm{Al}_{2} \mathrm{O}_{3}$ ratios that are greater than chondritic, an observation that is best explained by majoritic garnet fractionation in a deep magma ocean (Bertka and Holloway, 1994; Bertka and Fei, 1997). However, the $~ 3.7 \mathrm{Ga}$ Gusev basalts have chondritic $\mathrm{CaO} / \mathrm{Al}_{2} \mathrm{O}_{3}$ ratios; along with other elemental ratios and concentrations, it is apparent that Gusev basalts and basaltic martian meteorites are derived from different mantle sources (McSween et al., 2004; Schmidt and McCoy, 2010). This discrepancy suggests that different regions of the martian mantle may have undergone different processes and calls into question a global magma ocean. If the mantle inferred from martian meteorites is not applicable to all of Mars, then estimates of mantle water contents derived from martian meteorites (e.g., Mysen et al., 1998; McCubbin et al., 2010) may also have limited applicability.

The main shortcomings of the over 55 unpaired martian meteorites are listed in Table 2. Any version of MSR must enable science in the area of this objective over and above what is possible using the martian meteorites.

2.2.3.1.1. Sample types of interest-C1. Igneous rocks would provide the best possible samples for age determination by radiogenic isotopes and for constraining the martian interior with trace elements, isotopic composition, and other analyses. Locations should be identified from composition and geomorphological context, for example, igneous 
Table 2. Shortcomings of Martian Meteorites as Representative/High-Priority Samples of Mars

\begin{tabular}{|c|c|}
\hline No. & Shortcomings of Martian Meteorites \\
\hline 1 & $\begin{array}{l}\text { Sampled out of context } \\
\text { - Launched from unknown sites, with unknown geological context on Mars by impact }\end{array}$ \\
\hline 2 & $\begin{array}{l}\text { Inadequate sampling of the Noachian and Hesperian } \\
\text { - With one exception, all are }<1.4 \mathrm{Ga} \text {, i.e., Amazonian }\end{array}$ \\
\hline 3 & $\begin{array}{l}\text { They are all shocked to varying degrees } \\
\text { - Experienced peak pressures of up to } 60 \mathrm{GPa} \text {; affects mineralogy and radiogenic isotopes }\end{array}$ \\
\hline 4 & $\begin{array}{l}\text { No evolved igneous compositions } \\
\text { - All are mafic to ultramafic }\end{array}$ \\
\hline 5 & $\begin{array}{l}\text { Most have been affected by liquid-crystal fractionation } \\
\text { - Limitation for determining compositions of their parental melts }\end{array}$ \\
\hline 6 & $\begin{array}{l}\text { Orientations relative to planetary surface are not known } \\
\text { - Not usable for magnetic studies }\end{array}$ \\
\hline 7 & All have been subjected to deep space radiation (affects some isotopes) \\
\hline 8 & Do not provide ground truth for orbital or surface missions \\
\hline 9 & All (but especially the finds) have been affected by some form of terrestrial alteration and/or contamination \\
\hline
\end{tabular}

intrusions or lava flows. Samples should be as unaltered and unweathered as possible so as to preserve the igneous texture and distribution of elements as set during crystallization; preservation of these aspects would be more important than other attributes such as compositional or age diversity. In-place sampling would be strongly preferred over float. Samples from float that could be tied to a local outcrop ("subcrop") or volcanic vent (e.g., pyroclastic materials) are strongly preferred over other rocks that may have been carried some distance by impact (and therefore are also more likely to have witnessed high shock effects). Samples should be located so that their stratigraphic age could be narrowly constrained, in order for their absolute ages, once determined on Earth, to provide constraints on the stratigraphic sequence from which they were obtained (and contribute to Objective B2). Age diversity would be less important than obtaining unaltered or unweathered samples with known stratigraphic context.

The following sample types are of priority interest for achieving this objective:

1. Ancient igneous rocks. There is no sample of the presumed primitive crust of Mars among the meteorites, although studies of martian meteorites indicate that silicate differentiation on Mars occurred by $4.51 \mathrm{Ga}$ (Borg et al., 2003) and crystallization of a magma ocean, and subsequent overturn, may have been responsible for establishing initial reservoirs, including a primitive crust (e.g., Elkins-Tanton et al., 2005; Blinova and Herd, 2009). Thus, early Noachian igneous rocks would be preferable for this purpose. Similarly, there are no samples of late Noachian to early Hesperian among the martian meteorites. Within these constraints, the following rock types are preferred:

a. Noncumulus basalt (e.g., chilled flow margin). Two examples of noncumulus basalts are present among the martian meteorites. Of these, Yamato 980459, a glassy, olivine-bearing basalt whose bulk composition matches that of its presumed parent melt (Musselwhite et al., 2006), is thought to represent a primitive melt from the martian mantle. Samples similar to Yamato 980459 would be preferable in order to remove some of the difficulties in reconstructing parental melts, for example, from melt inclusions. A suite of samples from chilled flow margin to flow interior would be highly desirable.

b. Ultramafic (including xenoliths of possible mantle or lower crustal origin). The majority of the martian meteorites are mafic to ultramafic in composition. None of the meteorites has compositions and mineralogy consistent with an ultramafic mantle xenolith or a sample of the lower crust. Although the sampling of such a rock type would be fortuitous (see sampling strategies below), such samples would provide insights into the mineralogy of the martian interior, allow tests of mantle mineralogy inferred from meteorites (Bertka and Fei, 1997), and place further constraints on the thermal state of the martian interior, in conjunction with geophysical data (Khan and Connolly, 2008). A sample suite that included mantle xenoliths(s) with basaltic melt counterpart(s) would provide links between mantle source composition and derived melt(s) and would be desired but not required. A cumulate ultramafic rock would provide insights into crystallization processes in a martian magma chamber and processes of magma evolution on Mars in general.

c. Evolved igneous compositions. The range of igneous compositions on Mars is limited; martian meteorites are limited to basalts + ultramafic rocks, whereas igneous rocks at Gusev are primarily basalts, with some compositions falling in the picrobasalt and tephrite fields (McSween et al., 2009). More felsic compositions have been identified with thermal emission spectroscopy that potentially include evolved, granitic rocks (Bandfield et al., 2004; Christensen et al., 2005). A sample of an evolved igneous rock would provide significant insights into the potential range of igneous compositions that may be produced by melting of the martian interior. A sample suite of igneous rocks, for example, related by liquid line of descent would be desired but not required.

2. Young volcanic rocks. The youngest martian meteorite is $\sim 170 \mathrm{Ma}$ (Nyquist et al., 2001); nonetheless, orbital observations show clues for volcanic activity in the last hundred million years and possibly even in the last million years (Neukum et al., 2004). Although examples are generally older, IR spectroscopic observations suggest that volcanic eruptives of diverse compositions may exist on Mars 
(Christensen et al., 2005). If a young volcanic rock could be sampled, it would provide significant insight into the range of volcanic processes and the diversity of mantle sources planet-wide through time. Furthermore, a young volcanic sample would provide information on the martian core if paleomagnetic measurements were made. Finally, such a sample would be an excellent target for evaluating the longevity and history of water on Mars because our current models suggest that water has only existed episodically on its surface in the recent to modern times.

Significance of sampling for magnetic studies. The magnetic history of Mars is a fundamental question that relates to the geological evolution of the planet as a whole (e.g., Lillis et al., 2008; Weiss et al., 2008). The ability to quantify the duration and magnitude of Mars' magnetic dynamo would provide novel insights into the thermal evolution of the martian interior. Paleomagnetic measurements could also be used to test the hypothesis that Mars experienced plate tectonics and true polar wander, and as a tool for field geology (e.g., using magnetostratigraphy as a chronometer to correlate rock sequences). The ideal suite that would likely address this question would include Noachian to Hesperian samples, because Mars' core is generally thought to have been active during this time frame (although it is not entirely clear when the dynamo died). If only one sample could be selected, it should be as old as possible in order to get a sample that records paleomagnetism and allows for measurement of the intensity of the magnetic field at that time point (as determined from radiometric dating of the same sample). Any early Noachian rock would likely preserve a record of the dynamo. It would not be necessary to target high-magnetization areas (as identified from MGS results; Acuña et al., 2001), although those areas would be of interest to explain the strong fields observed.

The most useful rocks for this purpose would be igneous because they tend to contain primary igneous magnetic minerals (e.g., titanomagnetite and pyrrhotite). However, sedimentary rocks, especially those containing iron oxide minerals (e.g., hematite, maghemite, and goethite) as cement, may also be useful and would provide the advantage of paleo-orientation (using bedding) and various field tests that could demonstrate primary magnetization (fold test and conglomerate test).

Although the intensity of the magnetic field would provide significant information, the fraction of science value achieved with intensity data alone would be less than that which could be achieved with oriented samples. To determine the absolute direction of the ancient martian magnetic field with only a few samples, it would be extremely important that the orientation of each sample with respect to the martian surface be recorded. The direction could be used to test the dynamo hypothesis, to test hypotheses that Mars experienced plate tectonics and true polar wander, and to conduct magnetostratigraphy.

Implications for the sampling system. The importance of inplace sampling would be very high for achieving Objective C1. Samples collected within a known geological context, including those of a local (i.e., outcrop), regional, and planetary scale, would provide the primary advantage over martian meteorites and allow for the information obtained from their study on Earth to be directly related to the specific phase of
Mars' planetary evolution they represent. Sampling of rocks from probable Noachian terrane would enable the presumed age to be tested by radiometric dating on Earth. Sampling of rocks from outcrop, within the context provided by outcropscale characterization, would allow for selection of igneous rocks that have undergone lower levels of shock metamorphism and selection for compositional diversity. Therefore, the implications on the sampling system might include

(1) Need to visit Noachian to early Hesperian igneous outcrop, targeting those least affected by alteration, weathering, or impact shock metamorphism. Implication: capacity to get beneath weathering rinds to fresh samples; capacity to assess level of shock metamorphism.

(2) Target rocks that exhibit mineralogical or textural characteristics that suggest rapid cooling from a melt (e.g., quenched flow margin). Implication: capacity to characterize texture at sufficient spatial resolution.

(3) Samples of opportunity: During the traverses of the sampling rover, we should constantly check for exotic blocks that would allow us to further expand the range of rock types sampled. Ultramafic xenoliths in outcrop (e.g., within a basaltic unit) would also represent a sample of opportunity. Implication: capacity to assess compositional diversity and macroscale textural variations at a distance.

(4) The implication for paleomagnetic samples: capacity to sample such that the orientation with respect to Mars' surface would be preserved. Samples would need to be isolated from magnetic fields (ideally less than $0.2 \mathrm{mT}$; Weiss, written communication, 2011).

2.2.3.2. Objective C2. Constrain the origin and evolution of the martian atmosphere, accounting for its elemental and isotopic composition with all inert species. Understanding the evolution of the martian atmosphere is essential to explaining the occurrence of liquid water on the surface in Mars' early history, and that in turn influences conclusions on the habitability of the planet. Existence of liquid water on the martian surface, other than short-lived concentrated brines, requires higher atmospheric temperatures and pressures than present-day conditions. Although precisely how the warmer conditions were achieved remains unknown, a thicker $\mathrm{CO}_{2} / \mathrm{H}_{2} \mathrm{O}$ atmosphere (released from the martian interior by extensive volcanism in the early Noachian) is implied. Evidence from surface features on Mars suggests, however, that this atmosphere, and thus persistent surface liquid water, had largely disappeared by the end of the Noachian, which led to the dry and low-pressure conditions observed on Mars today.

Models of formation, evolution, and loss of the martian atmosphere are based for the most part on the isotopic compositions of noble gases (Pepin, 2000, 2006). Currently available data on some atmospheric components are from in situ analyses by the Viking landers, but the isotopic compositions of neon, krypton, and xenon are exclusively from analyses of the martian meteorites, in particular EETA79001 (see review by Swindle, 2002). The latter contains unfractionated atmosphere in its impact shock glass (Table 3).

Important, but so far unsolved questions require high precision composition data of all noble gases and their isotopes, which would be only achievable with a returned 


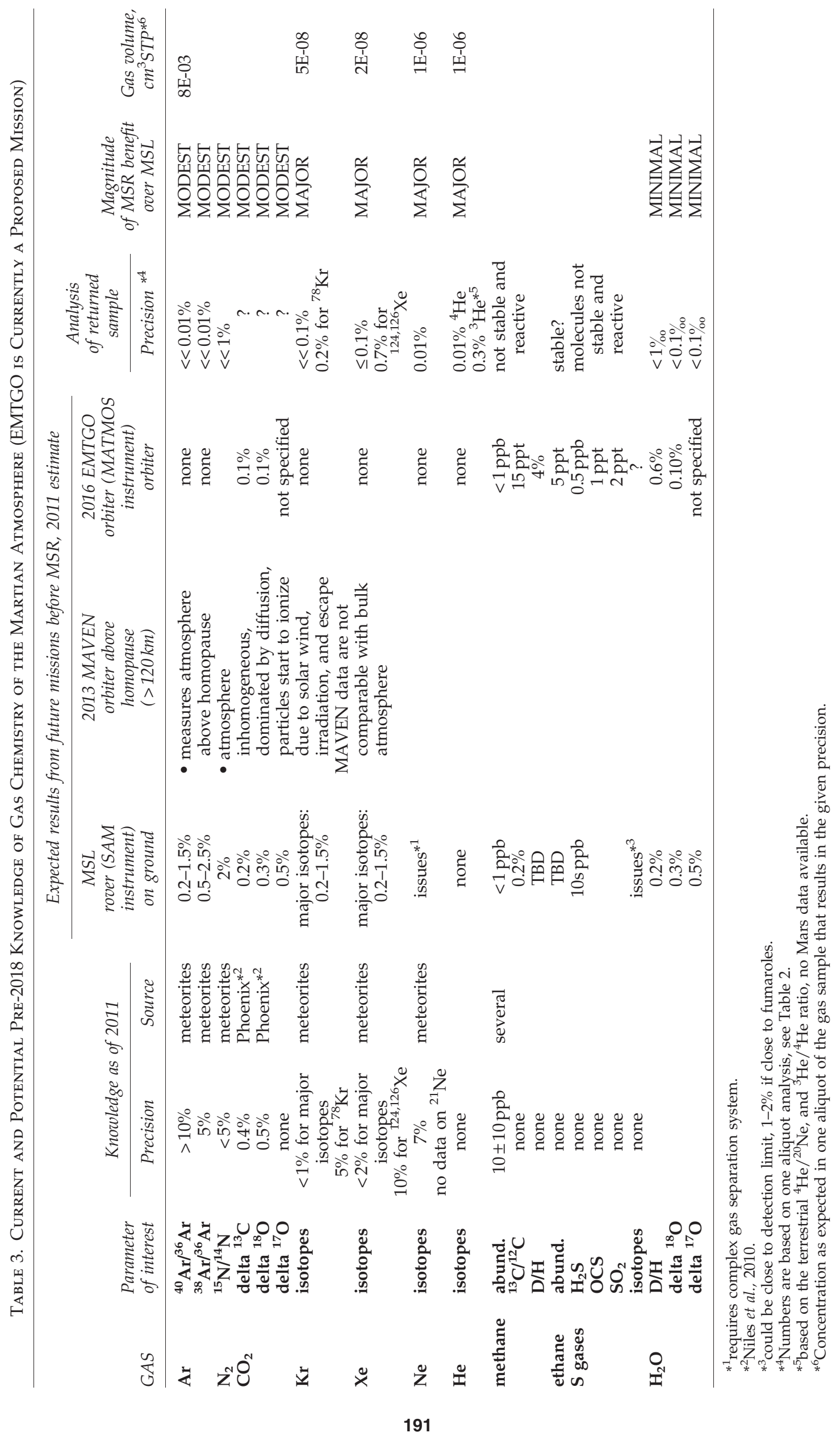




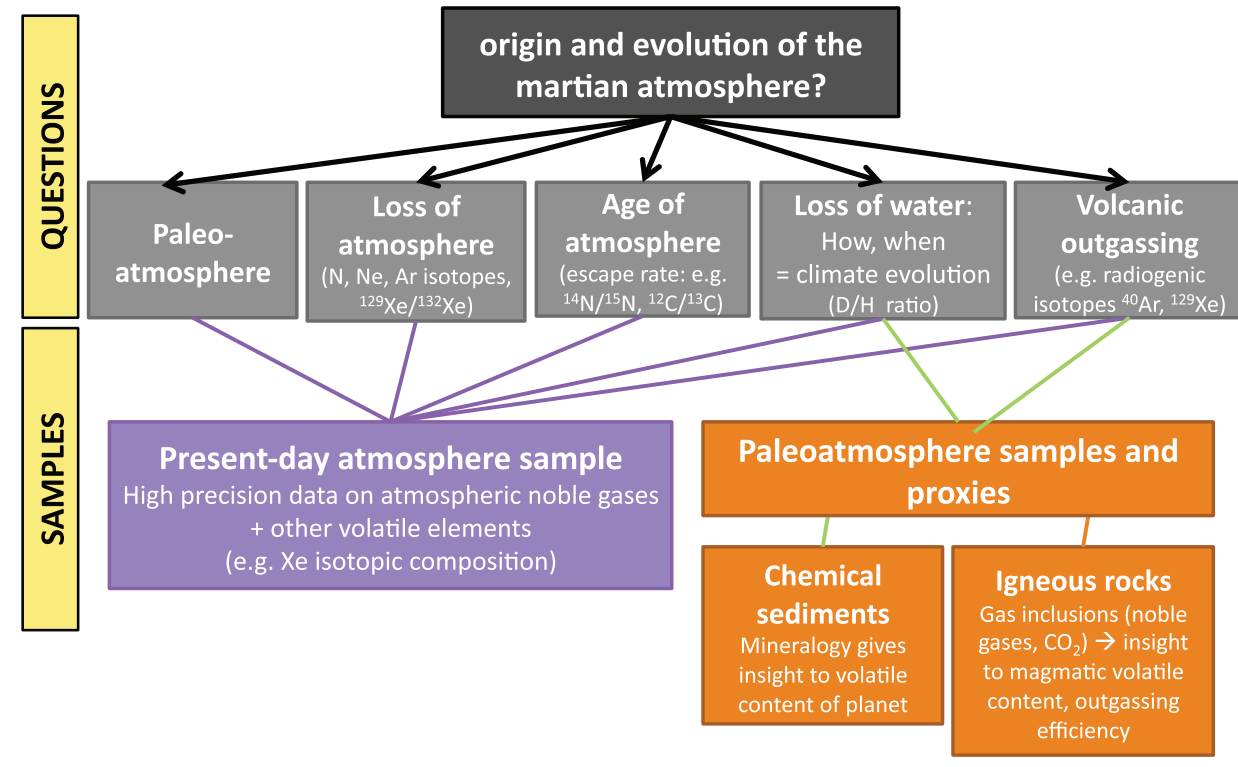

FIG. 3. Overall scientific objectives of atmospheric gas samples. Color images available online at www.liebertonline .com/ast

atmospheric gas sample analyzed in terrestrial laboratories. Those questions involve (a) the formation of the atmosphere: primordial versus volcanic outgassing versus late veneer (volatiles delivered from outside, for example, by comets); (b) duration of the thicker warmer atmosphere; (c) age of the atmosphere: young (continuous outgassing) versus old (primordial); and finally (d) the cause of the atmospheric loss: impact erosion during the late heavy bombardment versus a steady atmospheric escape after the disappearance of a global magnetic field (Fig. 3).

Atmospheric noble gas science is mainly based on EETA79001 impact glass, but interpretation of meteorite data is complicated due to possible mixing of different noble gas components. Spallogenic products mask the light noble gases $(\mathrm{He}, \mathrm{Ne}, \mathrm{Ar}$ ) and the minor isotopes of $\mathrm{Kr}$ and $\mathrm{Xe}$, and a significant correction is required for the more abundant isotopes. Krypton and xenon in meteorite finds are also altered due to adsorption of terrestrial atmospheric gases. Other martian meteorites revealed the presence of multiple components: martian interior, a soil component, and a paleoatmospheric signature (see review by Swindle, 2002).

Future in situ analyses by the MSL rover Curiosity, the 2013 MAVEN, and the proposed 2016 ExoMars Trace Gas Orbiter (EMTGO) orbiters would analyze the trace gases $\left(\mathrm{CO}_{2}, \mathrm{H}_{2} \mathrm{O}, \mathrm{O}_{2}, \mathrm{CH}_{4}, \mathrm{~N}\right.$, sulfur molecules, $\mathrm{C}_{2} \mathrm{H}_{6}$, etc. $)$ and their $\mathrm{H}, \mathrm{C}, \mathrm{N}, \mathrm{O}, \mathrm{S}$ isotopic compositions with high precision (written communications: MSL, Paul R. Mahaffy, 2010; MAVEN, Bruce Jakosky, 2010; EMTGO, Mark Allen, 2010). Table 4 summarizes the strengths and limitations of both in situ measurements and returned samples. The former provide high spatial and temporal resolution data of the atmospheric composition and are also capable of analyzing nonstable gas molecules (e.g., sulfur molecules, $\mathrm{CH}_{4}, \mathrm{H}_{2}$ ). A returned gas sample, in contrast, would allow highprecision analyses of all stable components, including the minor isotopes of the noble gases and other stable species (as $\mathrm{CO}_{2}$ and $\mathrm{N}_{2}$ ). As a consequence of the limitations of the meteorite data and orbital measurements, the analysis of

Table 4. Strengths and Limitations of IN Situ and Return SAmpling of Mars Atmosphere (EMTGO is Currently a Proposed Mission)

\begin{tabular}{|c|c|c|c|}
\hline & Mission/samples & Strengths & Limitations \\
\hline \multirow[t]{4}{*}{ in situ } & Viking & $\begin{array}{l}\text { Noble gases (abundances, isotopic } \\
\text { compositions) }\end{array}$ & Uncertainties (10-20\%) \\
\hline & MSL & $\begin{array}{l}\text { - Capture of seasonal and spatial } \\
\text { variability }\end{array}$ & $\begin{array}{l}\text { No } \mathrm{He}, \mathrm{Ne} \text {, minor } \mathrm{Kr} \text { and } \mathrm{Xe} \text { isotopes with } \\
\text { large uncertainties, no spatial resolution }\end{array}$ \\
\hline & MAVEN & $\begin{array}{l}\text { - Abundances + isotopic composition of } \\
\text { nonstable molecules (as sulfur }\end{array}$ & $\begin{array}{l}\text { Composition of the uppermost } \\
\text { atmosphere (ionized particles, escape) }\end{array}$ \\
\hline & EMTGO/MATMOS & $\begin{array}{l}\text { molecules, } \mathrm{CH}_{4} \text { (if it exists) } \\
\text { - High precision on abundant species }\end{array}$ & No noble gases \\
\hline \multirow[t]{2}{*}{$\begin{array}{l}\text { returned } \\
\text { sample }\end{array}$} & SNC meteorites & $\begin{array}{l}\text { Heavy noble gases, = basis for many } \\
\text { atmospheric models } \\
\text { High precision }\end{array}$ & $\begin{array}{l}\text { Significant cosmogenic contribution to all } \\
\text { noble gases, He too low, no control on } \\
\text { when gas was introduced nor on } \\
\text { different gas components }\end{array}$ \\
\hline & MSR & $\begin{array}{l}\text { All stable atmospheric species (noble } \\
\text { gases, } \mathrm{CO}_{2}, \mathrm{~N} \text { ) } \\
\text { High precision also for minor isotopes }\end{array}$ & $\begin{array}{l}\text { No spatial or temporal variability } \\
\text { Nonstable atmospheric species do not } \\
\text { survive }\end{array}$ \\
\hline
\end{tabular}


noble gases would be a major objective for a sample return mission (Table 3).

FINDING \#5: We will have at least a partial understanding of the geochemistry of most major components of the martian atmosphere prior to the proposed receipt of samples on Earth. The area in which we anticipate still having the most significant unresolved questions is in the noble gas isotopes.

FINDING \#6: The noble gases in martian meteorites are often mixtures of different components that introduce ambiguities in interpretation of measured data. A direct sample of the atmosphere would remove this ambiguity.

Detailed open questions related to martian noble gas geochemistry:

A better ${ }^{36} \mathrm{Ar} /{ }^{38} \mathrm{Ar}$ value would help to constrain models of atmospheric loss. The martian atmosphere is the only one in the Solar System that shows a significant deviation from solar ${ }^{36} \mathrm{Ar} /{ }^{38} \mathrm{Ar}$. Presumably, this is due to atmospheric loss of the lighter isotope, which is somewhat mitigated by mantle outgassing. Resulting loss rates should be in conjunction with the Ne isotopic composition, the ${ }^{14} \mathrm{~N} /{ }^{15} \mathrm{~N}$, and the ${ }^{12} \mathrm{C} /{ }^{13} \mathrm{C}$ in $\mathrm{CO}_{2}$ composition.

Ne and He isotopic composition (and abundance) to constrain atmospheric evolution and mantle degassing. Neon abundances and its isotopic composition in the martian atmosphere are still poorly known. Both ${ }^{20} \mathrm{Ne}$ and ${ }^{22} \mathrm{Ne}$ are difficult to measure at Mars because of serious ${ }^{40} \mathrm{Ar}$ and ${ }^{44} \mathrm{CO}_{2}$ interferences. In martian meteorites, isotopes are masked by cosmogenic Ne. MSL will not be able to produce highprecision data due to the lack of a gas separation mechanism. The Mars Atmospheric Trace Molecule Occultation Spectrometer (MATMOS) instrument proposed for EMTGO would not measure noble gases. Thus, all Ne science would be deferred to MSR.

The He abundance and isotopic composition in the martian atmosphere is unknown as it is entirely masked by cosmogenic contribution in meteorite samples. The He isotopic composition in fluid inclusions in igneous rocks that are not exposed to galactic cosmic rays (GCR) would deliver valuable information on the degassing history of Mars.

Combined atmospheric Kr and Xe signatures. Krypton and Xe and their isotopic compositions are key in determining the starting composition of the volatiles on Mars. However, modeling the evolution of the martian atmosphere is seriously affected by the absence or imprecision of critical data. Xenon in the martian atmosphere is either of solar or chondritic origin. However, whereas the ${ }^{129} \mathrm{Xe}$ suggests significant martian interior outgassing, missing heavy $\mathrm{Xe}$ isotopes related to ${ }^{244} \mathrm{Pu}$ fission $\left({ }^{131-136} \mathrm{Xe}\right)$ indicate the opposite. Precise and accurate measurements of the rare ${ }^{124-126} \mathrm{Xe}$ and ${ }^{128} \mathrm{Xe}$ isotopes would not only reveal the extent of mass fractionation of the starting composition but also the degassing history of Mars (Pepin, 2006).

The $\mathrm{Kr}$ isotopic composition of Mars' atmosphere may be either solar (Pepin, 1991) or fractionated in favor of light isotopes (Garrison and Bogard, 1998). Presumably, atmospheric $\mathrm{Kr}$ has been replenished by degassing after early atmospheric loss. Additionally, elevated amounts of ${ }^{80,82} \mathrm{Kr}$ indicate neutron capture by $\mathrm{Br}$. However, it is unclear whether this is a feature of the rock (i.e., a soil component) in which the $\mathrm{Kr}$ was measured or an atmospheric signature. It is clear that a model of the origin and evolution of the martian atmosphere has to include and coherently explain the isotopic compositions and abundances of all noble gases and other volatiles.

FINDING \#7: A key to understanding the origin and evolution of the martian atmosphere would be to measure the isotope systematics of each of the noble gases and construct a model that is consistent for all of them.

Seasonal variability of the present martian atmosphere. The present martian atmospheric pressure seasonally varies by about $20 \%$, due to the polar $\mathrm{CO}_{2}$ ice cap formation and sublimation. Swindle et al. (2009) proposed that a substantial fraction of $\mathrm{Xe}$ and less of the $\mathrm{Kr}$ and Ar are incorporated into polar clathrates (or perhaps also in ice). Accordingly, the seasonal formation or dissociation of a small amount of clathrates could change the atmospheric $\mathrm{Kr} / \mathrm{Xe}$ ratio and would probably have some effect on the isotopic composition of Xe. Detectable seasonal changes of $\mathrm{C}$ and $\mathrm{O}$ isotopic composition due to sublimation/condensation of $\mathrm{CO}_{2}$ ice are also considered possible (Mark Allen, personal communication, 2011). Respective fractionation of the $\mathrm{Xe}$ and $\mathrm{Kr}$ isotopes, either due to $\mathrm{CO}_{2}$ ice or clathrate formation, thus could not be ruled out. No further studies have been carried out (Tim Swindle, Bob Pepin, Oliver Mousis, personal communications, 2011). However, MSL may be able to address this question by monitoring the $\mathrm{Kr} / \mathrm{Xe}$ and a Xe isotopic ratio (best ${ }^{129} \mathrm{Xe} /{ }^{136} \mathrm{Xe}$ ) over 1 martian year. If significant variations are found, two atmospheric gas samples should be taken, one at pressure minimum and the other at pressure maximum. The high-low pressure pair could assess the extent of seasonal clathrate formation (and the incorporation of Xe). Secondly, it could reveal differences driven by other mechanism in the atmosphere (Bob Pepin, personal communication, 2011). Finally, the second sample would also mitigate risk by serving as a backup if one sample is lost (e.g., on Mars due to sealing failure).

FINDING \#8: There would be scientific value in collecting atmospheric samples at two different seasons; however, although this is considered desired, it is not required. If it were possible to return more than one sample, we do not currently recognize a need to collect such samples from more than one location.

2.2.4. Aim D. Prepare for human exploration. The eventual human exploration of Mars would require information that could-and should-be acquired by means of sample return. The primary questions to be addressed by analysis of the first returned samples include the potential hazards and potential resources to be found in the surface and near subsurface.

2.2.4.1. Objective D1. Assess potential environmental hazards to future human exploration. For a human mission to the martian surface, it is considered impossible to break the chain of contact with Mars (see, for example, Drake, 2009). This means that uncontained martian material would be transported back to Earth via the astronauts and their equipment. Planning for such a mission would therefore be 
critically dependent on prior information about potential biohazards in the soil/dust. A sample of the ubiquitous airfall dust would indicate whether such material constitutes a biological, mechanical, or electrostatic hazard to future exploration (see MEPAG, 2010). A surface soil sample would demonstrate the potential hazard of this widespread material and its capacity to either harbor or destroy microbial life. A sample of soil from the shallow (1-2 m) subsurface would show whether this environment is significantly different from the surface as a potential niche for life.

1. Airfall dust. Airfall dust is one of the most widespread surface types on Mars. It is present, to a greater or lesser degree, in virtually every region, and it can be lifted, injected into the atmosphere, and transported locally, regionally, and globally by mechanisms that range from local dust devils to global dust storms. Human explorers to any location on the planet would be exposed to this dust. It could prove to be a biohazard as well as a hazard to equipment due to mechanical or electrostatic interactions. Experience from Spirit and Opportunity shows that patches of pure airfall dust of sufficient size and thickness to sample are present along both traverse paths. Such natural deposits are more common along the Spirit traverse, which is in a dustier part of Mars. Passive collection devices may be required to sample pure airfall materials (see MEPAG ND-SAG, 2008), rather than being dependent on encountering sampleable geological deposits. In the absence of easily accessible pure airfall dust deposits, for the purpose of this objective, samples of soil with admixed airfall dust may be sufficient to achieve the objective. Because the dust circulates globally, a sample returned from any location is thought to be sufficiently representative of all of martian dust (e.g., Christensen et al., 2004a, 2004b).

2. Surface soil. Surface soil has been analyzed by each of the previous lander and rover missions. Results from Viking and Phoenix suggest that much of this material contains a strong oxidizing agent that may rapidly destroy organic material. This chemical component, in concert with the temperature extremes, desiccation, and radiation environment, indicate that the martian surface is extremely hostile to life. However, analysis of a returned sample of surface soil would more closely define the chemical and mineralogical composition as well as the biohazard potential of this widespread material.

3. Shallow subsurface soil. The logic of MEPAG's Goal IV is that the martian regolith would need to be tested for biohazards down to the expected depth of disturbance by the future human mission (MEPAG, 2010). However, it is not clear what that depth should be. The maximum depth of wheel disturbance by MER was approximately the diameter of the wheels (although when this occurred it was unintentional and created significant mobility challenges for the rover). Given that a rover designed to carry humans would be significantly heavier, the depth of disturbance could be greater (perhaps as much as $50 \mathrm{~cm}$ ?). The depth of human footprints would unlikely be more than a few centimeters. If the human mission includes mining, road building, or some other "Mars-moving" operation, the depth could be a small number of meters. These factors could be considered when determining the depth from which a soil sample should be collected.
Importantly, the physical and chemical conditions that make the martian surface extremely hostile to life are significantly less in the shallow subsurface ( $2 \mathrm{~m}$ or less). Such a finding would have profound effects on future Mars exploration, both robotic and human. The shallow subsurface soil could become an important exploration target for possible future life-detection missions. In addition, landing systems and surface operations could certainly reach this depth - either intentionally or accidentally-and would have to be designed with possible forward and backward planetary protection in mind.

Although a "deep" regolith sample would strategically be very important, and is highly recommended by the E2EiSAG, the proposed 2018 rover may not have the capability to deliver such a sample to the cache, which would be necessary for it to be returned to Earth.

2.2.4.2. Objective D2. Evaluate potential critical resources for future human explorers. One of the implications of NASA Design Reference Architecture 5.0 (Drake, 2009) is that perhaps the single greatest factor that would change the planning basis of a potential future human mission to Mars for the better is the identification of recoverable hydrogen resources at the martian surface. MEPAG (2010) followed up by proposing several specific investigations that could contribute to this end (see Investigation IV.A.2A). Although carbon and oxygen are important resources to support a human stay at the martian surface, they could be readily available from the $\mathrm{CO}_{2}$-rich martian atmosphere. Hydrogen (or equivalently, water), however, is not sufficiently abundant in the atmosphere. Since water would be required for several purposes, it either would need to be acquired in situ or delivered from Earth-for the latter option, the masses would be especially daunting for long-stay missions. For a hydrogen/water resource on Mars to be of practical use, it would need to be present at the surface or contained within the shallow regolith in order to be compatible with anticipated extraterrestrial mining and processing technology.

Although water ice would be a valuable resource for hydrogen, and certainly is present in the midlatitudes and high latitudes (e.g., Boynton et al., 2002; Head et al., 2003), landing site (especially latitude), planetary protection, and sample preservation considerations all make the return of ice samples impractical for the first sample return mission. An alternative comes from minerals that contain $\mathrm{H}_{2} \mathrm{O}$ or $\mathrm{OH}$, which have been recognized both from orbit and on the surface such as within phyllosilicates, zeolites, and hydrated sulfates (e.g., Christensen et al., 2004b; Bibring et al., 2006; Murchie et al., 2009). In most cases, the areal extent of these minerals appears limited to relatively small portions of the planet. However, because such places would be of high scientific interest due to their association with past water, it is likely that they would be an attraction both for returned sample science and for future human explorers. But it is also likely that the concentrations of hydrated minerals recognized from orbital remote sensing occur in rocks or outcrops, which would make extraction more challenging. Ideally, a regolith source of hydrated minerals would be found.

Airfall dust is known to contain one or more $\mathrm{H}_{2} \mathrm{O}$-bearing phases (e.g., Murchie et al., 2000; Christensen et al., 2004a; Ruff, 2004). Given its ubiquity across the planet, establishing the abundance and extractability of water from such a sample would be important. Hydrated mineral phases have 
Table 5. Criteria Used by the E2E-ISAG to Prioritize Science Objectives

Top-level Prioritization Criterion:
Ways in which returned

sample analysis adds

value (subcriteria)
Examples:
1. Addresses science questions of high intrinsic priority

2. Address questions for which little meaningful progress can be made without sample return

The value of the incremental knowledge to be gained by returned sample analysis
3. Answers have higher definitiveness

4. Addresses questions for which there is an advantage if the analytical approach can be discovery-responsive (analysis pathway not limited by instrument payload). a) Instrumentation hard/impossible to miniaturize or make robust enough for interplanetary flight

b) Scale of investigation not amenable for in situ

c) Sample prep impossibly complex

a) Better accuracy, precision

b) Results confirmed by alternate methods been recognized in light-toned soils at Gusev Crater (e.g., Yen et al., 2008) and probably exist in many other locations. Generic dark-toned soils likely contain some fraction of airfall dust with its hydrated component but, in bulk, appear to be much less hydrated than the dust in isolation as well as light-toned soils (e.g., Yen et al., 2005, 2008).

\subsection{Prioritization of scientific objectives}

A key part of the E2E task was to "prioritize a reference set of 'campaign-level' science objectives." Given the science objectives described above, the E2E team developed a toplevel criterion and a set of subcriteria to guide the prioritization (Table 5). Since the chief aim of the proposed MSR Campaign would be to return martian samples to Earth for detailed study, the key criteria for prioritizing objectives is the value of the incremental knowledge to be gained by returned sample analysis. This value is judged by the degree to which sample analysis could address the highest-priority questions in Mars science, as most recently summarized by MEPAG (2010) and the NRC (2011). The value of returned samples is highest if they address questions for which little progress could otherwise be made due to instrument or sample preparation complexity, or to the scale of the investigation. Laboratory analyses generally have notable advantages over in situ analyses in terms of precision and accuracy, and laboratory results could be confirmed by alternate methods. In addition, laboratory instruments and techniques could be adapted to discoveries much more easily than instruments that are locked into a spacecraft payload.

Using these criteria, we have ranked the science objectives in priority order (Table 6). It is worth noting that, as a multidisciplinary team, the members of the E2E committee have diverse interests and perspective, and there was a certain amount of variation in how scientists from different disciplines viewed these priorities. However, despite that, the placement of Objectives A1 (life) and C1 (planetary evolution) as the top two priorities (in that order) was strongly felt by the scientists from almost all disciplines represented on the team.

As per the arguments discussed above in Section 2.2.1.1, the search for evidence of extant life cannot be effectively prioritized with the same criteria. The importance of this objective is superimposed on all the others.

Table 6. Summary of Science Objectives Defined by the E2E-iSAG in Priority Order

\begin{tabular}{|c|c|c|}
\hline Priority & $\begin{array}{c}\text { Objective } \\
\text { Reference \# }\end{array}$ & Objective Description \\
\hline 1 & A1 & $\begin{array}{l}\text { Critically assess any evidence for past life or its chemical precursors, and place detailed } \\
\text { constraints on the past habitability and the potential for preservation of the signs of life. }\end{array}$ \\
\hline 2 & C1 & $\begin{array}{l}\text { Quantitatively constrain the age, context and processes of accretion, early differentiation, } \\
\text { and magmatic and magnetic history of Mars. }\end{array}$ \\
\hline 3 & B1 & Reconstruct the history of surface and near-surface processes involving water. \\
\hline 4 & B3 & Constrain the magnitude, nature, timing, and origin of past planet-wide climate change. \\
\hline 5 & D1 & Assess potential environmental hazards to future human exploration. \\
\hline 6 & B2 & $\begin{array}{l}\text { Assess the history and significance of surface modifying processes, including, but not } \\
\text { limited to: impact, photochemical, volcanic, and aeolian. }\end{array}$ \\
\hline 7 & $\mathrm{C} 2$ & $\begin{array}{l}\text { Constrain the origin and evolution of the martian atmosphere, accounting for its elemental } \\
\text { and isotopic composition with all inert species. }\end{array}$ \\
\hline 8 & D2 & Evaluate potential critical resources for future human explorers. \\
\hline ADDITIONAL & A2 & Determine if the surface and near-surface materials contain evidence of extant life. \\
\hline
\end{tabular}




\section{Achieving the Scientific Objectives-Some Considerations Involving Collecting Samples from a Field Area}

\subsection{Relationship between field and sample science}

For some laboratory studies of samples an associated field context is available, and for some studies it is not (e.g., meteorite studies). The former is always better constrained and therefore more useful than the latter, and for the kinds of complex astrobiology/geology questions posed in this report, field context would be required. As applied to MSR, this is what distinguishes a "grab-and-go" version of MSR (MEPAG, 2002) from a selected-sample version of MSR (NRC, 2011). Robust interpretation of even the most sophisticated laboratory analyses of geological specimens ultimately relies on the quality and extent of observations of the field relationships used to select samples. This fundamental insight was aptly summarized by the legendary sedimentary geologist, Francis Pettijohn: "For only by a firsthand acquaintance with the primary phenomena of geology, obtainable only by field study, can significant research be distinguished from the trivial" (Pettijohn, 1984, p 248). Accordingly, for the MSR Campaign to obtain the greatest information, it is considered crucial that samples be collected such that their geological context is well understood. Some of this context could be characterized from orbital data obtained prior to landing, but many details would only be obtained by in situ study at, and around, the sites of collection.

FINDING \#9: The integration of field and sample science would be critical to answering complex geological/astrobiological questions.

\subsection{Information hierarchy}

Figure 4 illustrates the time-honored approach to answer scientific questions that involves the use of both a selected field site and laboratory analysis data-such studies are ex-

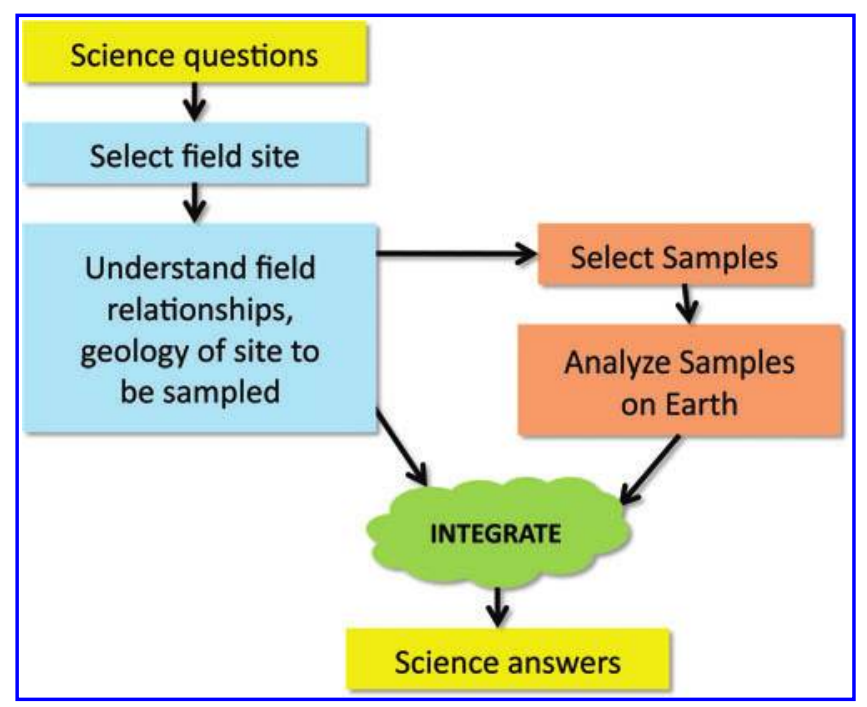

FIG. 4. Schematic flow chart outlining the roles of samples and geological field characterization in the evaluation of scientific questions. Color images available online at www.liebertonline.com/ast tremely common in the natural sciences. It begins with wellposed scientific questions and selection of a field site for sampling that best addresses those questions. Geological relationships of the site are characterized through a variety of approaches (e.g., lithological mapping, stratigraphic traverses, textural observations). Appropriate sample suites are then collected on the basis of those field relationships. Ideally, this is done in a sequential manner, but on Mars (and most investigations on Earth) logistics would impose a more iterative process between characterizing geological context and selecting samples. Samples returned from the field are examined and analyzed in laboratories, and finally results from both the field and laboratory are then integrated to obtain "answers" that respond to the original questions.

It is virtually axiomatic to science that any investigation of a scientific question will lead to new, unanticipated questions and to new ways to pose the original question that provide better insights. Accordingly, another advantage of characterizing geological context as thoroughly as practicable during sample selection is that it would allow for unanticipated and revised questions to be addressed without necessarily returning to the sampling site, a critical asset for planetary missions.

FINDING \#10: Putting together effective sample suites would require collecting information in the field on many more geological targets than the number of samples eventually collected.

\subsection{Collect early, exchange later}

In addressing geological questions that involve coupled field work and sample-based laboratory studies, there is commonly a tension between collecting potentially valuable samples when they are encountered and waiting to understand the geology better before starting to fill up the sample collection. In principle, it would be ideal to complete all geological mapping first and then use that information to decide which samples would be most important. However, field studies are rarely carried out this way because of the extreme inefficiency of returning to localities a second time to sample. Therefore, samples are typically collected as the field survey is being carried out. Some of the earlier samples will inevitably turn out to be less valuable than those encountered later (especially because the geology is better understood), but the time-honored way of solving this on Earth is to toss the earlier, less valuable samples out of the backpack and replace them with later-collected more valuable and appropriate samples. This capability greatly reduces the pressure on sample collection decision-making early in the traverse and allows the geologist to benefit from his or her increasing knowledge as fieldwork progresses.

On Mars, the inefficiency of returning to outcrops a second time to collect samples would be unacceptable given the limited lifetime and traverse capability of a rover. Thus, the importance of building the collection "as you go" is even higher on Mars than on Earth. Therefore, as discussed above, to support effective decision-making, the capability to swap out samples is judged to be extremely important. The collective judgment of the E2E-iSAG team is that an excess sampling capability of $25 \%$ should be sufficient to address this need. That is, $25 \%$ of the core tubes could be replaced 
with cores of higher value for the final suite that would come back to Earth.

FINDING \#11: The scientific value of the collection would be significantly enhanced if the sampling rover had the capability and lifetime needed to replace at least $25 \%$ of samples collected earlier with samples of higher value collected later.

\subsection{The importance of rock and soil sample suites}

It is possible for individual, unrelated samples to provide useful data. However, most rocks record the effects of multiple geological processes, and these effects can only be unraveled uniquely by studying multiple samples in which the effects differ. Single samples could be likened to individual snapshots that do not provide information about gradients of change in time and space. It is common with such single datum samples not to know how they are positioned in the evolution of time and within the variability of environments. They provide limited help in identifying the geological and environmental context. The evaluation of complex systems like those anticipated for the MSR Campaign would be particularly context-dependent. It is critical to decipher the geological changes that remain impressed in the stratigraphic record of outcrops and other terranes by analysis of a series of samples. Therefore, it would be necessary to collect suites that comprise a series of samples that are genetically connected in order to build a vision of planetary environmental evolution through time. This finding reinforces a key conclusion previously made by the MEPAG ND-SAG (2008).

FINDING \#12: Sample collections organized around one or more sample suites, designed using key geological relationships, maximize their potential for answering scientific questions.

\section{Achieving the Proposed Scientific Objectives- Samples Required/Desired}

\subsection{Integrated priorities for rock samples}

Rock sample types are listed below in priority order with a single prioritization criterion: potential value to achieving the objectives in Table 6. Category 1 samples are given the highest priority because of their importance for addressing the possibility of past life and issues related to climate change and the history of water. Category 2 samples (fresh igneous rocks), the next highest in priority, are important for absolute dating and understanding the evolution of the interior of the planet.

Category 1A. Subaqueous sediments or hydrothermal sediments. These primary sample target types were rated to be of highest priority in the search for life due to the fact that these have the greatest potential for preservation of biological signatures, as well as prebiotic chemical signatures and observations that help establish an abiotic baseline. In addition, subaqueous and hydrothermal sedimentary deposits have the maximum potential to provide contextual information on the depositional environment, habitability, and preservation potential, based on the preservation of information in order and sequence (clearly recording past spatialtemporal relationships) in the outcrop. They were rated of equal priority primarily to ensure that a sampling of multiple environments that might have suitably hosted a broad spectrum of possible modes of life was captured. This includes not only a focus on sedimentary formations formed subaqueously that may preserve surface-based life but also includes sample types that encompass the possibility of life based on chemoautotrophic principles (similar to those discovered at hydrothermal vents, hot springs, and emerging subsurface groundwaters on Earth) (Chapelle et al., 2002; Kelley et al., 2005; Lin et al., 2006; Chivian et al., 2008; Proskurowski et al., 2008).

Category 1B. Hydrothermally and low-temperature fluidaltered rocks. The distinction between these is described above in Section 2.2.1.1. As with the samples in Category 1A samples, these fluid-altered rocks are also relevant to $\mathrm{Ob}$ jectives A1, C1, C2, and in a minor way to B2 (for fluid inclusions). Category $1 \mathrm{~A}$ samples are of higher priority than those of Category 1B due to the inherently higher potential for preservation of both biotic and chemical signatures as well as for the higher potential for preserving contextual information regarding the timing and paleoenvironmental sequence and context for life. In addition, a sample suite derived from a sedimentary record would be more useful to Objectives C1 and C2 because of the added value of good temporal context.

Category 2. Unaltered igneous rocks. This sample type is required to achieve Objective $\mathrm{C} 1$, the second-highest-priority objective in Table 6 . In contrast to the Category 1 samples, the less altered the sample by water-related processes, the better. It is strongly preferred that the sample would be free of shock effects and so should be sampled in place rather than from float, but the sampling rover could encounter lithological exotics on the martian surface that have been blasted in from somewhere else that would be well worth sampling. Although this sample type is crucial to Objective $\mathrm{C} 1$ and is of minor importance to C2 (because of fluid inclusions), it is of little importance to the other objectives.

Category 3. Regolith samples. These are vital for understanding the hazards that surface material may present to human exploration (Objective D1) and the opportunities that may exist for human resources (Objective D2). Regolith samples are also of scientific interest for insights into surface/atmosphere interactions and interactions with the space environment (Objective B3). Finally, if the regolith samples contain exotic fragments of rock that improve the diversity of the returned sample collection, they could be of great value to Objectives A1, B1, B2, C1, and C2.

Category 4. Atmosphere. This sample type is definitively required to achieve Objective $\mathrm{C} 2$, which is important, but in a relative sense is ranked priority \#7 of 8 (Table 6). Fortunately, it would be an easy sample to acquire-no sample selectivity would be required. There is no value of this sample type to the other objectives.

4.1.1. Considerations in designing the suites of rock samples. It is not a general principle that all geological questions would require sample suites. Some kinds of questions could be addressed with single samples. However, the four highest-priority scientific questions posed for the MSR Campaign (Section 2) are at a level of complexity that 
could not credibly be approached without analysis of multiple samples organized into suites that represent the geological relationships present in the field area. For these questions, the differences between samples could be as important, or more so, than the absolute character of single samples (also reinforcing the findings of the MEPAG NDSAG, 2008).

4.1.2. Possible/probable variations in rock we need to be prepared to detect and sample. It is not possible to describe in advance the details of optimal sample suites that need to be collected because this would depend on the kinds of rocks that are available to be sampled, which, in turn, would depend on the landing site, the traverse plan, and many other factors. However, it is possible to predict the kinds of natural variations we would encounter that are of direct relevance to the objectives. The ability to observe and measure these kinds of variations is crucial for interpreting the site geology and locating the optimum suites of samples at that site for achieving the science objectives. In the scientific objectives discussed above, there are some clear commonalities in terms of the kinds of samples that would benefit each of the objectives (Section 2.3), and it is important that we have an understanding of the possible variations we may encounter related to those objectives as it imposes constraints on the rover capabilities at any realistic landing site and traverse plan. For this reason, we list here some of the possible geological variations that may be encountered and are of direct relevance to the proposed objectives, and some possible ways to configure sample suites to capture those variations:

Possible/Probable natural variations on Mars related to sedimentary/hydrothermal rocks

- Facies and microfacies in a sedimentary deposit

- Physical variations in a mineral phase: texture, fabric, crystal habit and residence of the mineral in veins, layers, cement, clasts, concretions, etc.

- Physical variations in clast size, shape, distribution, and spatial arrangement

- Inferred paleosalinity gradient in a salt mineral assemblage

- Variations in organic matter: host mineralogy, concentration, spatial arrangement in relation to context

- Sedimentary structures and textures and correlated mineralogical variations

- Mineral transitions across a zone of alteration

- Sequence of vein-fill deposits or changes in fracture density, or both

- Proximal-distal trends (laterally and vertically) in mineralogy or cement at a hydrothermal vent, or both

Some initial sample selection factors for sedimentary/hydrothermal sample suites

- Rocks that have high potential to preserve organic molecules, including biological remains

- Rocks that enable interpretation of paleoenvironmental conditions

- Rocks from different stratigraphic positions that span potential changes in past climate

- Rocks that exhibit mineralogical or textural characteristics that may be microbially influenced or have high probability of supporting biological activity (e.g., chemical gradients) and preserving it (e.g., rapid deposition)

- Rocks whose compositions are likely to provide constraints on the composition of ancient surface or subsurface waters

- Rocks that represent essential variations within a hydrothermal system, such as water/rock ratio, temperature, and fluid chemistry

Possible/Probable natural variation on Mars related to igneous rocks

- Petrologic character: range of compositions (e.g., ultramafic to mafic), variation in mineralogical or trace element properties, or both

- Age (although in the field this could only be hypothesized based on context)

- Type and intensity of aqueous alteration and degree of weathering

- Igneous setting: intrusive, extrusive; local setting within the igneous body

- Grain size, chemical variation in minerals

- Degree of impact shock metamorphism, including brecciation

Some initial sample selection factors for igneous sample suites

- Rocks of probable Noachian age that have known stratigraphic context

- Rocks that best preserve primary igneous character: least affected by alteration, weathering, or impact shock metamorphism

- Rocks that span potential variations in bulk composition

- Rocks that contain xenolithic clasts

- Rocks that exhibit mineralogical or textural characteristics that suggest rapid cooling from a melt (e.g., quenched flow margin)

FINDING \#13: The proposed rover would need to be equipped with scientific capabilities to observe and measure the kinds of geological features (and variations therein) that would enable us to recognize the geological settings and sampling targets that are needed to meet the science objectives.

4.1.3. Scientific value of a subsurface rock sample. The capability to return one or more samples from $\sim 2 \mathrm{~m}$ depth would be extremely valuable. Modeling (e.g., Dartnell et al., 2007) shows that subsurface rock would have been protected from GCR by overlying rock/regolith for at least some of its history. The same is true for the potential role of solar UV on organics buried by ejecta from impact events (Cockell et al., 2002) or in the martian soil (Stalport et al., 2009). Organic matter therefore has a much greater chance of being preserved in subsurface materials than in surface samples. In addition, UV oxidation of $\mathrm{Fe}^{2+}$ to $\mathrm{Fe}^{3+}$ (e.g., Burns, 1993; Morris et al., 2010) would be less prevalent at depth. Moreover, since subsurface rocks are protected from cosmic rays, any fluid inclusions would potentially have better-preserved noble gas isotopic compositions. Therefore, subsurface igneous fluid/melt inclusions could provide information on Mars' degassing history, and fluid inclusions in sedimentary or aqueously altered rocks could provide clues on the aqueous history of Mars. 
Table 7. Approximate Duration and Number of Rocks/Soils Studied in Detail During Various Science "CAMpaigns" of the MER SPIRIt Rover

\begin{tabular}{|c|c|c|c|}
\hline Campaign & Description & Duration & Samples \\
\hline Hydrothermal System & Exposures of opaline silica-bearing rocks and soils in the Eastern Valley & $\sim 100$ sols & $\sim 10$ \\
\hline Alkaline Igneous Province & Igneous rocks preserved on the Gusev plains and Columbia Hills & $\sim 60$ sols & 7 \\
\hline Pyroclastic Deposit & $\begin{array}{l}2 \text { m Home Plate section of altered pyroclastics and reworked aeolian } \\
\text { sediments }\end{array}$ & $\sim 30$ sols & 7 \\
\hline Targets of Opportunity & $\begin{array}{l}\text { Examples of distinctive rock classes encountered during ascent } \\
\text { of Husband Hill }\end{array}$ & $\sim 200$ sols & $\sim 8-10$ \\
\hline TOTALS & & $\sim 390$ sols & $\sim 32-34$ \\
\hline
\end{tabular}

FINDING \#14: While the MSR Campaign could be justified using near-surface samples alone, the capability to return subsurface samples from a depth of up to $2 \mathrm{~m}$ would be scientifically valuable because of the possibility of enhanced preservation of organics.

4.1.4. Number of rock samples desired/required. In any geological study, the number of samples required to address a well-defined problem is always a difficult issue; depending on the question, the number may lie anywhere between a single sample (e.g., an internal isochron of an igneous rock) and many hundreds, if not thousands, of samples (e.g., highresolution climate change records). What are appropriate numbers of rock samples to address the science objectives (Table 6) and sampling priorities (Section 2.3 of this report) of the MSR Campaign?

Considerable insight has been gained from the experience of the MER mission (see references below). The Spirit rover operated for more than 6 years in a target-rich environment in Gusev Crater and examined more than 75 rocks in situ. Within this extended period of time, the rover carried out a number of reasonably well-defined "campaigns" that lasted on the order of weeks to months and were designed to investigate, in detail, major discoveries. These campaigns, in turn, provide some guidance to the number of samples that might be required for MSR.

Four relevant Spirit campaigns bear some broad similarity to the science objectives defined for the MSR Campaign:

(1) A hydrothermal system defined by the exposures of opaline silica-bearing rocks and soils in the Eastern Valley near Home Plate (Squyres et al., 2008; Ruff et al., 2011);

(2) An alkaline igneous province defined by alkaline volcanic rocks in the Columbia Hills of Gusev Crater that had sources similar to the Adirondack-class volcanic rocks found in the Gusev plains (McSween et al., 2006);

(3) The Home Plate deposit comprising a $2 \mathrm{~m}$ layered section of poorly sorted pyroclastics debris overlain by well-sorted sandstones thought to represent aeolian reworking of the pyroclastics (Squyres et al., 2007);

(4) The ascent of Husband Hill, during which the Spirit rover traversed a target-rich geological setting of layered rocks and encountered numerous lithologies that could be considered as "targets of opportunity" (Arvidson et al., 2006; Squyres et al., 2006).

Table 7 summarizes the details of these campaigns and the number of samples each examined in some detail in order to address the hypothesis that initiated the detailed study. Also shown is our best estimate of campaign durations. Durations are difficult to estimate because, in many cases, campaigns were initiated some time after the initial observations, multiple issues were being addressed simultaneously, unrelated traverses were interspersed, and so forth. Nevertheless, an overall conclusion from this analysis is that on the order of $\sim 30-35$ mixed rock and soil samples would be sufficient to achieve the major science objectives of the MSR Campaign and that the time required to obtain such samples would likely take on the order of a martian year or less (Fig. 5).

FINDING \#15: For the kinds of landing sites of interest to the MSR Campaign, the number of high-priority rock samples is estimated to be $\sim 30-35$. This reaffirms a key finding of the ND-SAG, who recommended 28 rock samples as a minimum number.

\subsection{Considerations related to the number and type of regolith samples}

4.2.2. Regolith-sampling implications. Evidence from MER and orbital surveys suggests that soil (i.e., the fine component of regolith) has some attributes that are similar everywhere on Mars, either as a result of global dispersion or common formation processes (Yen et al., 2005). Though approximately $25 \%$ of soil is reported to consist of alteration products, it is nonetheless thought that martian soil is

Number

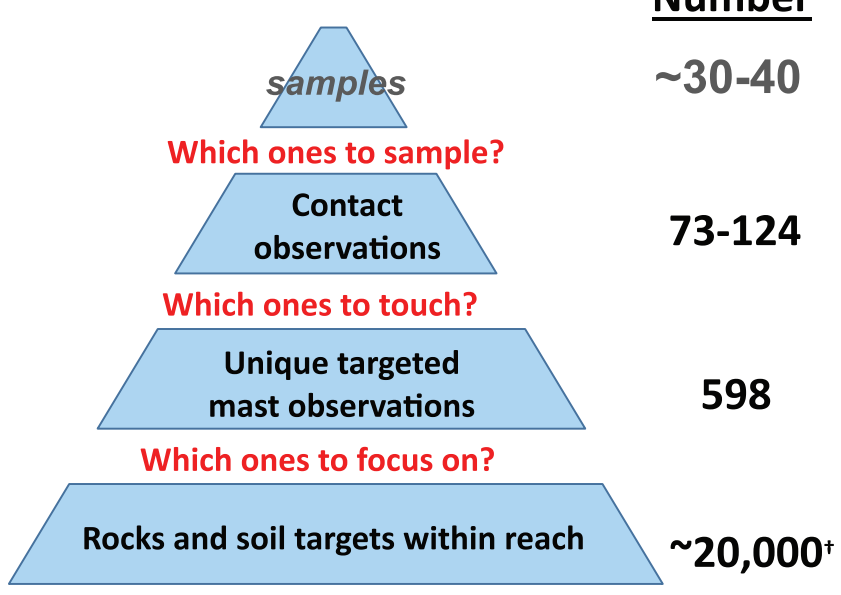

FIG. 5. Schematic diagram, based on lessons learned from the MER Spirit, illustrating the hierarchical nature of obtaining sufficient geological context information in order to select the best samples for return to Earth. Estimates of targets within reach $(\dagger)$ is based on number of rocks readily visible to Spirit while traversing during the first martian year. Color images available online at www.liebertonline.com/ast 
primarily abraded rock, with altered rock being overrepresented due to a greater probability of abrasion (McSween et al., 2010). Nonetheless, there are reasons other than redundancy to select samples from multiple locations, including an expectation of photochemical products such as perchlorate in the near surface (Hecht et al., 2009) and the fact that the likelihood of finding biomarkers is significantly different within and below the diurnal thermal skin depth. Depending on the nature of the landing site and the sampling capability available, it is possible that as many as 3-4 samples could be useful for the different kinds of investigations, and in any case, at least one regolith sample is considered essential.

A surface soil sample should be obtained from the top $5 \mathrm{~cm}$. The sampling should be performed at a distance from the lander sufficient to avoid any physical effects (e.g., erosion or removal of fines) or chemical contamination due to the lander itself or its thrusters and landing gear. Although a single sample of generic soil may be sufficient to satisfy most objectives, airfall dust may not be distinguishable from the soil's finest fraction. Ideally, airfall dust would be sampled separately from soil. The MERs demonstrated that relatively thick accumulations of airfall dust (several millimeters thick) are present at both sites, but mobility was required to reach them. It is unlikely that such accumulations would be accessible from the MAV lander, and its descent rockets would likely winnow out the finest fraction. Therefore, we suggest that within the MSR Campaign such samples must be collected by the caching rover.

Other regolith samples like light-toned soils are considered samples of opportunity enabled by mobility. Such samples could be identified with remote sensing and in situ instruments on board the rover. In all cases, identifying regolith samples of interest would require no additional measurement capability beyond that needed to sample rocks. However, the generally loose, particulate nature of regolith material may place different demands on the sampling method.

A key point is that the diversity and degree of scientific interest of different kinds of granular samples at the 2018 landing site would not be known until the proposed mission lands and enters into its exploration. By far the most useful implementation would be one in which the sampling and caching capability could be used for either rock or regolith and then allow the science team of the future to decide, based on what is available to them, the relative proportions of these two sample types.

FINDING \#16: Multiple regolith samples collected from the 2018 rover would be significantly more valuable than one or more regolith samples collected from the immobile MSR-L.

FINDING \#17: No measurement capability beyond that required to select and document the rock samples would be needed for regolith samples. However, the physical acquisition and packaging of a granular sample may have important implications on the sampling/ packaging system.

\subsection{Considerations related to the number and type of gas samples}

4.3.1. Integrated priorities for gas samples. The most important priority is at least one sample of atmospheric gas of sufficient size (see Section 6 for sizing analysis). If it is possible to do so, a second gas sample would be scientifically useful and is considered desired. If two atmospheric samples are taken, one should be collected at the atmospheric pressure minimum and the other at the pressure maximum (see 2.2.3.2.).

4.3.2. Atmospheric gas-sampling implications. Atmospheric temperature and pressure should be measured at the time of sampling. The gas container should maintain a gastight (ultrahigh-vacuum-quality) seal. Additionally, a double valve (both ultrahigh vacuum) would be scientifically valuable in order to assess the quality of the sample at the time it is received in the lab. Having two valves would also simplify later sample handling.

4.3.3. The importance of rock/mineral samples with fluid or melt inclusions. Noble gases (particularly radiogenic isotopes) as well as the other volatiles (e.g., $\mathrm{H}_{2} \mathrm{O}, \mathrm{CO}_{2}, \mathrm{~N}_{2}$ ) in fluid inclusions in igneous rocks could reveal the magmatic volatile content of the martian interior and allow for testing of postulated contents derived from martian meteorite studies (e.g., McSween et al., 2001; Jones, 2007; Filiberto and Treiman, 2009; McCubbin et al., 2010). Analysis of these gases thus would allow for assessment of the planet's outgassing efficiency, which in turn has implications on the formation and evolution of the atmosphere. Fluid inclusions in sedimentary rocks could reveal the composition of water from which the sediment was deposited.

However, due to the missing magnetic field, the thin atmosphere, and a low erosion and crustal recycling rate, many surface rocks have been more or less continuously exposed to GCR for much of martian history. For this reason, apart from "fresh" crater excavations, the light noble gas content $(\mathrm{He}, \mathrm{Ne}, \mathrm{Ar})$ in surface rocks on Mars is presumably completely masked, and the heavy noble gases (Kr, $\mathrm{Xe}$ ) severely altered. For comparison, a $90 \%$ correction has to be applied to the light Xe isotopes in the Nakhla meteorite due to irradiation by GCR during its 12-million-year journey in space (Mathew and Marti, 2002). A drill sample ( $\sim 2 \mathrm{~m}$ ) would be of advantage to facilitate data interpretation, even though it would not solve the problem completely when not drilled into a solid piece of rock. (Any particle of the regolith can be considered as being within the upper 1 or $2 \mathrm{~m}$, the penetration depth of GCR, at some time.)

FINDING \#18: Gas geochemistry objectives would require the analysis of a dedicated martian atmosphere sample. The analysis of the fluid inclusions in solid samples would provide additional valuable information on volatile content in martian interior and outgassing efficiency. However, the noble gas composition could be masked or severely altered by GCR irradiation, thus either freshly excavated crater material or a $\sim 2 \mathrm{~m}$ drill core sample would be required.

\section{Where on Mars Might It Be Possible to Obtain the Samples Needed to Achieve the Proposed Objectives of MSR?}

\subsection{Establishing a reference landing site set}

Meeting the scientific objectives of the MSR Campaign (Table 6) is dependent on whether there are places on Mars 
Table 8. Threshold and Qualifying Criteria for Reference Landing Sites

Threshold Geological Criteria

1. Presence of subaqueous sediments or hydrothermal sediments (equal $1^{\text {st }}$ priority), OR hydrothermally altered rocks or low- $T$ fluid-altered rocks (equal $2^{\text {nd }}$ priority)

2. Presence of aqueous phases (e.g., phyllosilicates, carbonates, sulfates.) in outcrop

3. Noachian/Hesperian age based on stratigraphic relations and/or crater counts

4. Presence of igneous rocks with known stratigraphic relations, of any age, to be identified by primary minerals

Starter List of Qualifying Geological Criteria

1. Morphological criteria for standing bodies of water and/or fluvial activity (deltaic deposits, shorelines, etc.)

2. Assemblages of secondary minerals of any age

3. Presence of former water ice, glacial activity or its deposits

4. Igneous rocks of Noachian age corresponding to unaltered primitive crust, better if including exhumed megabreccia

5. Volcanic unit of Hesperian or Amazonian age well-defined by crater counts and well-identified by morphology and/ or mineralogy

6. Probability of samples of opportunity (ejecta breccia, mantle xenoliths, etc.)

7. Potential for resources for future human mission

that host the desired materials for sampling and whether a rover could access and sample them. To establish the potential for at least one site on Mars to both satisfy the highestpriority MSR science objectives and have the capacity to land, we conducted a process to identify potentially viable candidates. Our goal was to produce a reference landing set, consisting of several reference landing sites, each of which could potentially meet the objectives of Table 6 and illustrate a range of scientific and engineering attributes that span the trade space of interest and could be quantitatively evaluated.

The search for reference landing sites began with a review of the $\sim 60$ landing sites proposed for the MSL mission (Grant et al., 2011) and $\sim 25$ additional community-proposed landing sites identified for possible future missions (originating through a 2010 Future Landing Sites call). Although the overall objectives for the MSR Campaign would differ from those of these other missions, they were viewed as a good starting point because of some overlap in science objectives and because many of the sites considered for other missions are partially to nearly completely covered by high-resolution spatial and spectral resolution data (e.g., from Mars Reconnaissance Orbiter, Mars Express, and Odyssey). We chose sites with an eye toward providing a range of characteristics for both science and engineering that could be used to help define landing and roving requirements. Sites with substantial existing image coverage were favored because such data enable meaningful engineering studies of the MSR Campaign EDL system requirements for accessing the eventual landing site.

FINDING \#19: In order to end up with at least one acceptable site after science and engineering constraints are evaluated, it is necessary to begin the scientific selection process with a reasonable array of candidates.

To screen candidate sites for the MSR Campaign, we used the four threshold criteria listed in Table 8. These threshold criteria relate primarily to the inferred depositional setting and age of the rocks considered to be of highest priority for sample return and include the strong desire for the presence of igneous rocks. Although additional preliminary qualifying criteria were identified, ranging from morphological evidence of setting to the age of volcanic units to be accessed (Table 9), these were not used in the E2E analysis process. (The job of prioritizing candidate landing sites needs to be done through a community-based process, separate from the E2E process.) Because the reference sites are not intended to serve as a short list for the actual mission, the more relaxed constraints allowed us to define reasonable science and engineering criteria for the proposed mission. A more rigorous and open landing site selection process is expected to follow. We anticipate that, once formal criteria are defined, a call for candidate sites would be made to the science community, which would initiate a comprehensive site selection process based on those employed for MER and MSL (e.g., Grant et al., 2004, 2011).

For reference landing set, the E2E-iSAG chose the following (Table 9): five candidates studied extensively during the MSL landing site selection process; the MER landing site in Gusev Crater; and an additional site at a relatively high northern latitude. We assumed a landing ellipse comparable to that of MSL $(\sim 20 \times 25 \mathrm{~km})$. Each of the sites appears to encompass all the threshold science criteria and define a latitude range of approximately $35^{\circ} \mathrm{N}$ to $15^{\circ} \mathrm{S}$, elevations ranging from $\sim-0.5 \mathrm{~km}$ (Mars Orbiter Laser AltimeterMOLA) and lower (Fig. 6), and a variety of relief (much of which was viewed as unacceptable for MSL touchdown criteria relating to hazard probability). A description of each of the reference sites follows.

\subsection{Brief descriptions of the reference landing sites}

The Nili Fossae region includes a diversity of hydrated minerals rarely obtained on Mars (e.g., Ehlmann et al., 2009). Three sites are proposed in this broad region, each with a different context and the presence of igneous rocks. Nili Fossae trough is located on Hesperian volcanic flows on the floor of the Nili graben. Locations favorable to astrobiology contain phyllosilicates in both layered and massive units (Mustard et al., 2009). More diversity is accessible east of the ellipse within the ejecta breccia of the crater Hargraves, which would enable a sampling of crustal rocks, both altered and unaltered, although not in place. About $200 \mathrm{~km}$ east of Nili trough, Jezero Crater is a paleolake identified by two delta fans (Fassett and Head, 2005). The fans contain hydrated minerals and possibly carbonates, and are likely to have collected material from the altered highlands (Ehlmann 
Table 9. Potential Landing Sites Identified by the E2E-ISAG

E2E Reference Landing sites

\begin{tabular}{|c|c|c|c|c|c|}
\hline \multirow[b]{2}{*}{ Site } & \multicolumn{3}{|c|}{ Center of Proposed Ellipse } & \multirow[b]{2}{*}{ The sedimentary/hydrothermal story } & \multirow[b]{2}{*}{ The igneous story } \\
\hline & $\begin{array}{l}\text { Lat } \\
\left({ }^{\circ} N\right)\end{array}$ & $\begin{array}{l}\text { Lon } \\
\left({ }^{\circ} E\right)\end{array}$ & $\begin{array}{l}\text { Elev. } \\
(\mathrm{km})\end{array}$ & & \\
\hline $\begin{array}{l}\text { Eastern } \\
\quad \text { Margaritifer } \\
\text { Terra }\end{array}$ & -5.6 & 354 & -1.3 & $\begin{array}{l}\text { In the channeled Noachian uplands south } \\
\text { of Meridiani Planum is a small, shallow } \\
\text { basin with an exposure of possible } \\
\text { chlorides stratigraphically overlain by } \\
\text { an eroding unit with very strong CRISM } \\
\text { and even TES signatures of } \\
\text { phyllosilicates. }\end{array}$ & $\begin{array}{l}\text { The rocks appear to be capped } \\
\text { by a basaltic unit of Noachian } \\
\text { age. }\end{array}$ \\
\hline Gusev Crater & -14 & 175 & -1.9 & $\begin{array}{l}\text { The Noachian-aged Columbia Hills } \\
\text { contain outcrops of opaline silica likely } \\
\text { produced from hot springs or geysers } \\
\text { and outcrops rich in Mg-Fe carbonates } \\
\text { likely precipitated from carbonate- } \\
\text { bearing solutions. Sulfate-rich soils and } \\
\text { outcrops also are present. }\end{array}$ & $\begin{array}{l}\text { Extensive unaltered Hesperian } \\
\text { olivine-rich basalts embay the } \\
\text { Noachian Columbia Hills. } \\
\text { Also present are several } \\
\text { different igneous rock types } \\
\text { with minimal alteration. }\end{array}$ \\
\hline Jezero Crater & 18.4 & 77.6 & -2.6 & $\begin{array}{l}\text { Delta with incorporated phyllosilicates } \\
\text { and carbonates along west margin of } \\
\text { crater. The crater formed in Noachian } \\
\text { olivine and pyroxene-rich crust. }\end{array}$ & $\begin{array}{l}\text { The crater floor has a more } \\
\text { recent unit likely Hesperian } \\
\text { that looks like fresh volcanic } \\
\text { flows. Would land on } \\
\text { volcanic and traverse to } \\
\text { delta. }\end{array}$ \\
\hline $\begin{array}{l}\text { Mawrth Valles } \\
\text { Site } 0\end{array}$ & 24.5 & 339 & -3 & $\begin{array}{l}\text { Layered } \mathrm{Al} \text { and Fe-Mg phyllosilicates in } \\
\text { poorly understood setting. Possible } \\
\text { mud volcano in the vicinity of ellipse. } \\
\text { Land on science for exobiology. }\end{array}$ & $\begin{array}{l}\text { Mafic material present in } \\
\text { ellipse, but may be partly } \\
\text { altered. Unaltered Hesperian } \\
\text { volcanic at } \sim 30 \mathrm{~km} \text {. }\end{array}$ \\
\hline $\begin{array}{l}\text { NE Syrtis } \\
\text { Major }\end{array}$ & 16.2 & 76.6 & -2.1 & $\begin{array}{l}\text { Extensive and diverse mineral } \\
\text { assemblages within ellipse in Hesperian } \\
\text { Syrtis Major volcanic region. Maybe } \\
\text { water-lain deposits or in situ alteration. } \\
\text { Likely go to required for all materials of } \\
\text { exobiological interest. }\end{array}$ & $\begin{array}{l}\text { Hesperian Syrtis Major volcanic } \\
\text { region. }\end{array}$ \\
\hline $\begin{array}{l}\text { Nili Fossae } \\
\text { Trough }\end{array}$ & 21 & 74.5 & -0.6 & $\begin{array}{l}\text { Widespread altered materials, as ejecta at } \\
\text { eastern side of ellipse, in place to west of } \\
\text { ellipse. }\end{array}$ & $\begin{array}{l}\text { Land on unaltered Hesperian } \\
\text { volcanic plain. }\end{array}$ \\
\hline $\begin{array}{l}\text { Ismenius } \\
\text { Cavus }\end{array}$ & 33.5 & 17 & $-\sim 3$ & $\begin{array}{l}\text { Single site to combine clay-bearing } \\
\text { paleolake sediments and current glacial } \\
\text { deposits. Three deltas at the same } \\
\text { elevation confirms paleolake } \\
\text { interpretation. }\end{array}$ & $\begin{array}{l}\text { Unaltered material may be } \\
\text { limited to dark sand, } \\
\text { unaltered bedrock outcrops } \\
\text { to be confirmed. }\end{array}$ \\
\hline
\end{tabular}

et al., 2008a, 2008b). The crater floor contains mafic minerals that show rough textures consistent with Hesperian-age volcanic flows. South of Jezero, the contact between altered highlands and unaltered Syrtis Major plains is very straight with a small scarp of lava flows that dominate altered highlands (Mangold et al., 2007). Landing on the plains at this NE Syrtis Major site would enable sampling of a welldefined volcanic unit with a go-to astrobiological objective inside the layered material beneath the lava flows and in the altered highlands. Sulfates may exist inside the layered material, and carbonates are present on this olivine-bearing unit of the Nili Fossae region (Mustard et al., 2007, 2009; Ehlmann et al., 2008b, 2009).

The Mawrth Vallis region is another area that displays evidence for substantial alteration, mainly in the form of phyllosilicates (e.g., Poulet et al., 2005; Loizeau et al., 2007,
2010; Bishop et al., 2008), which is considered of high interest for understanding the early martian environment and its astrobiological potential (Michalski et al., 2010). The site proposed is located northwest of the one that was under consideration for the MSL site selection in the final four list. In addition to layered materials containing $\mathrm{Al}$ clays and $\mathrm{Fe} / \mathrm{Mg}$ clays that crop out inside the ellipse, it also contains igneous materials, some that are likely present inside the ellipse, and some Hesperian-age volcanic flows in the western side of Oyama Crater that are reachable after a $20-30 \mathrm{~km}$ long traverse.

Eastern Margaritifer Terra is located in the channeled highlands of Noachian age, south of Meridiani Planum. The small basin where the ellipse is located contains phyllosilicates and possibly chlorides (Osterloo et al., 2008). The sequence of units exposed by erosion in this basin has an 


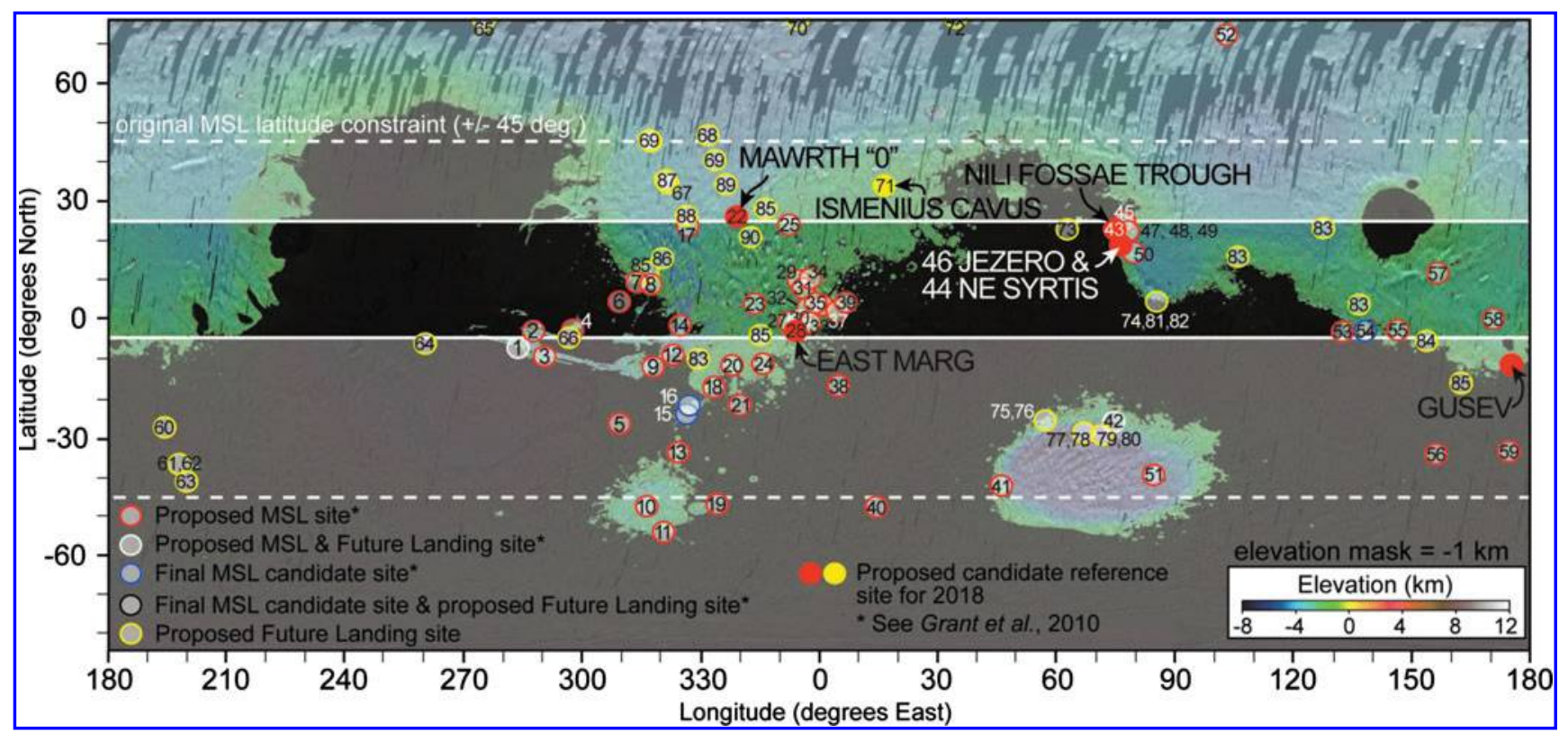

FIG. 6. Map showing landing sites considered by the E2E-iSAG analysis. Those proposed as candidate reference sites for the MSR Campaign are highlighted.

unaltered, basaltic unit at the top of the sequence that overlies a phyllosilicate-bearing unit, which in turn overlies a chloride-bearing unit at the base (Christensen et al., 2008). These units would be reachable inside the ellipse after a short traverse out of the ellipse to the east.

Gusev Crater has proved to be mineralogically diverse despite an apparent lack of evidence for the paleolake that motivated its selection as a MER landing site. Within the Columbia Hills in the center of Gusev, Spirit encountered soil and outcrops of nearly pure opaline silica, a clear manifestation of hydrothermal processes (e.g., Squyres et al., 2008) and entirely consistent with a hot spring or geyser origin (Ruff et al., 2011). Outcrops containing as much as $34 \%$ by weight $\mathrm{Mg}$-Fe carbonate were identified and perhaps represent another manifestation of hydrothermal processes (Morris et al., 2010). Sulfate-rich soils are yet another indication of the role of water. In addition to a range of igneous rocks within the Columbia Hills, they are surrounded by early Hesperian, olivine-rich flood basalts similar to lunar mare (Greeley et al., 2005). Gusev Crater provides both welldefined igneous units and a diversity of mineralogy and rocks formed in environments of astrobiological interest.

Ismenius Cavus is a 60 by $90 \mathrm{~km}$ elliptical trough located in the Ismenius Lacus region. It is the most challenging site proposed here, because it is at a high latitude $\left(34^{\circ} \mathrm{N}\right)$ and because mafic minerals are currently only observed as sands, even if volcanic plateaus surround the circular trough. The interest in this site is that it shares the presence of a paleolake attested to by three delta fans at the same elevation, phyllosilicates on layered deposits on the floor of the trough, and midlatitude glaciers on its side (Dehouck et al., 2010). The latter may open a unique opportunity to collect material from the Early Mars period and from Amazonian ice deposits in the same mission.

The seven sites identified here, in addition to containing reachable igneous rocks, have been chosen to be complementary and representative of different types of astro- biological interest: two paleolakes (Jezero and Ismenius Cavus), one ancient basin (E. Margaritifer), three hydrothermal sites (Nili Fossae trough, NE Syrtis Major, and Gusev), and one altered crust (Mawrth) with a variety of alteration or secondary minerals, or both (phyllosilicates, sulfates, chlorides, carbonates).

FINDING \#20: Among the $~ 85$ candidate landing sites that have been proposed by the community to date (for MSR and a range of possible future missions), at least seven potentially meet the preliminary list of MSR science criteria. However, further analysis of the sites would be needed to better evaluate their potential to meet the criteria.

\subsection{Implications of the reference landing set for the major EDL and mobility parameters}

The reference landing set (Table 9) has some important lessons for thinking about landing site-related planning.

- It is not easy to find sites where relatively unaltered igneous rocks are found close to sites selected for astrobiology where the rocks have been strongly altered by water. Retaining the igneous rock objective in the threshold criteria, therefore, likely would require rover mobility and lifetime to be large enough to access targets outside the landing ellipse. For example, among the seven reference sites proposed, most are likely "go-to" sites either for igneous rocks or astrobiology and would therefore require significant driving distance (i.e., $\sim 20 \mathrm{~km}$ ) and rover lifetime on the surface (assumed to be at least 1 martian year, although not evaluated in this study).

- Landing and traverse hazards identified previously (during the MSL landing site selection process) might in some cases be mitigated via incorporation of terrainrelative navigation and hazard detection and avoidance. 
This could allow us to land directly on the scientific targets at some of the reference sites. The ability to "land on" sites that are otherwise "go to" and where most or all science objectives could be met, could shorten the required traverse distances and mission lifetime-an extremely valuable benefit.

- Improvements in EDL that would narrow the diameter of the landing error ellipse (communicated to us by Chad Edwards, personal communication, 2011) could also have the effect of shortening drive times and possibly overall lifetime. Opportunities in this area should be pursued at a high level of priority.

An important caveat is that the seven reference sites are not known to be safe for the engineering criteria of the MSR Campaign (which would involve two landings at different times). The total number of potential sites would depend on how many of the sites in the reference set are ultimately judged to be acceptable to both science and engineering, and the reasons that any of them are rejected. However, the number of potential sites may never exceed 20-25. Consequently, all capabilities that could be added to the landing system to access additional sites should be considered.

Lastly, latitude limitations on the landing site, such as the $-5^{\circ} /+25^{\circ}$ constraint on the original ESA ExoMars rover (and the E2E-iSAG Charter), would also impact the number of potential sites that are responsive to all proposed objectives. Many southern latitudes below $-5^{\circ}$ display considerable evidence for aqueous alteration and putative lacustrine landforms, including sites such as Gusev Crater $\left(-14^{\circ}\right)$ and two of the final four MSL sites (Eberswalde and Holden at about $\left.-25^{\circ}\right)$. Northern latitudes in excess of $25^{\circ}\left(25-40^{\circ}\right)$ would also be of interest but may be less important than an opening of southern latitudes, especially because of the limited area of highland terrains above the $+25^{\circ}$ latitude. Thus, an enlargement of the southern latitude range, at least to $-15^{\circ}$ and perhaps to $-25^{\circ}$, would help to introduce a more significant number of candidate landing sites into consideration.

FINDING \#21: Three EDL/mobility factors would play a major role in the quality of the sample collection and therefore in determining the ultimate scientific return of the MSR Campaign:

- Whether the landing system could allow ellipse placement over terrain that is more hazardous than permitted for MSL

- Whether the ellipse could be reduced in size to allow placement between hazards.

- Whether the rover would have the capability to traverse to rocks outside the landing ellipse.

\section{Measurements on Returned Samples Required to Achieve the Proposed Objectives}

First, it is important to point out that, as the capabilities and priorities of laboratory science on Earth improve with time, the specific measurement opportunities and requirements will evolve. It is not our intent for this report to be the final word in returned sample measurement planning. Our goal is to document the current state of the art, with the presumption that any changes in the future would be an improvement on that.
As discussed above in Section 4, three general types of samples would be needed to achieve the scientific objectives in Table 6: rock samples, regolith samples, and one or more atmospheric gas samples. These three sample types would be subjected to different analysis pathways.

\subsection{Analysis flow for rock and regolith samples}

As per experience gained by the global curatorial community through processing of extraterrestrial samples returned by Apollo, Luna, Stardust, Genesis, and Hayabusa, the analysis of rock and regolith samples returned from Mars would be expected to follow a four-phase process:

I. Preliminary examination

II. Planetary protection assessments

III. Current allocations for scientific research

IV. Future allocations for scientific research

A significant part of the scientific interest in martian samples is because of their potential to contain evidence of past or present martian biology. For this reason, the second category above is far more important than it was for Stardust, Genesis, and Hayabusa. Steps I and II would therefore need to be done in a suitable containment facility, which for planning purposes has been referred to as the Sample Receiving Facility (Rummel et al., 2002; Beaty et al., 2009; and references therein). Depending on the outcome of the planetary protection assessments, it may be desired or required that Steps III and IV would also need to be done in containment. Another possible way to set up this planning, if the sample demands of the planetary protection assessments are too large, is to bypass Step II and carry out Steps III and IV in containment, treating the samples as if they were hazardous (although this approach would likely restrict the scope of experiments that could be performed, which would have significant negative consequences for the scientific investigations).

6.1.1. Preliminary examination. The aim of this phase of the analysis is to ascertain the external and internal characteristics of each returned sample by noninvasive and nondestructive techniques. The E2E-iSAG recommends that initial assessment utilize CAT (computer-aided tomographic) scanning, the advantage of which is that it would be possible to scan the specimens while they are held within the returned sample capsule. Some CAT scanners have substantial magnetic fields that could partially remagnetize the samples, so care would need to be taken to avoid this potential issue. The sample capsule itself would be scanned and then opened under conditions such that any evolved gases are collected. Each sample, still in its container, would be removed and then scanned again. Results from the scans would reveal the heterogeneity of each sample, including any layering, veins, clasts, pore spaces or fractures, and so on, and their gross mineralogy. Decisions could be made at this stage, even before the sample was removed from its container, about the subsampling strategy.

Following scanning, the samples would be removed from their containers. These samples would be weighed, photographed at a range of resolutions, and assigned unique designators for further tracking. The environmental conditions to which the samples would be exposed $(T, P$, gas 
chemistry, etc.) need further discussion. Exposed surfaces of all samples would be scanned with multispectral imaging and other nondestructive techniques to highlight regions of particular interest. Based on these examinations, subsamples would be separated for planetary protection analyses (Section 6.1.2). The remainder of each sample would be stored in clean, sealed containers pending the outcome of these analyses.

6.1.2. Planetary protection. The aim of this phase of the analysis is to determine whether there are indications of extant life in the samples and to assess any other biohazard potential of the sampled materials prior to their being made available to the research community. Such determination of potential hazard must be defined within internationally devised and accepted planetary protection protocols (COMPLEX, 2002; Rummel et al., 2002; NRC, 2009), mandated by international treaty (e.g., United Nations, 1966) and implemented by international agreements (Rummel et al., 2002; COSPAR, 2011).

A series of measurements would be carried out to assess two classes of potential hazard: (1) the possibility of replicating organisms and (2) the possibility that such organisms present a hazard to people who come into contact with the samples or to Earth's biosphere. These are commonly referred to as life-detection and biohazard (LD-BH) assessment. In addition, there would be a need to use the information learned from returned sample analysis to keep Mars-specific planetary protection policy (including both forward and backward protection) as up to date as possible.

The first draft of an MSR test protocol was prepared by Rummel et al. (2002), and it incorporates both destructive and nondestructive methodologies. Rummel et al. (2002) did not estimate the quantity of sample material needed to carry out the tests they described. However, they proposed a figure of $10 \%$ as a reasonable starting place to guide discussions [the same figure had been used earlier by DeVincenzi and Bagby (1981)]. For the present sample sizing calculations, we have therefore assumed that $1.5 \mathrm{~g}$ (of each sample) would be required for planetary protection-related testing, and that this material would not be available or suitable for scientific investigations after that (Table 10). When the next version of the test protocol is written, it may contain more or fewer tests requiring more or less sample material than the 2002 test protocol. As pointed out by Farmer et al. (NRC, 2009), the development of improved, less destructive, or nondestructive methods for LD-BH testing would be highly desirable and could reduce the amount of sample mass consumed.

It is important to recognize that some or all of the measurements designed to fulfill the requirements of planetary protection would also be of extremely high interest to the scientific objectives of the MSR Campaign (including the preparation for future human exploration, Objective D1). Some examples might include organic chemical analyses, $\mathrm{pH}$ measurements, characterization of any corrosive or other aggressive chemistries, determination of grain/dust particle sizes and distributions, and measurement of other petrographic properties. We also note that the use of material for planetary protection purposes does not necessarily preclude its use in later analyses for other purposes. Clearly, there is the opportunity and need to plan for these measurements in as coordinated a way as possible.
Sample and collection heterogeneity/diversity. An additional consideration is that it is possible that the quantity of material required for planetary protection testing would increase relative to the Rummel et al. (2002) guideline due to considerations relating to inter-sample and intra-sample heterogeneity and diversity. There are two issues related to the heterogeneity of the returned sample collection. As discussed in Section 4 of this report, the Mars surface operations team would be driven to maximize the diversity of the samples that make up the collection. The greater the diversity of the collection, the greater the chances of scientific discovery and the greater the collection's value. Since the MSR Campaign has a discovery-driven purpose, this is crucial. However, heterogeneity within individual samples could cause difficulties. Since all geological samples are heterogeneous, the proper question here is the scale and character of that heterogeneity (not the existence of heterogeneity itself). The issue is that centimeterscale heterogeneity in a $5-10 \mathrm{~cm}$ sample could lead to challenges in subdividing the sample and in having enough mass in each subsample type to carry out an integrated program of analysis. This may be especially problematic for planetary protection testing. While recognizing that some of the sample types of very high scientific interest (e.g., layered sedimentary rocks, hydrothermal rocks, breccia) commonly show heterogeneity at this scale, to first order, collecting samples that are relatively homogeneous at the scale of the sample may be preferable (and needs further discussion).

Given the above considerations, we arrive at the following assessment:

(i) Because of the hoped-for collection-level diversity, it is prudent to assume that at least one split from every sample would need to go through LD-BH testing. The necessary sample mass must be planned for.

(ii) If extant martian life is present in any of the samples, its spatial distribution may be heterogeneous, which would present a significant challenge to subsampling for planetary protection purposes (see NRC, 2009). However, we do not see a credible way to forecast this distribution or to understand the factors that control it, until the samples are studied on Earth. Different kinds of terrestrial life-forms, for example, respond in different ways to the heterogeneity available to them in rocks and soils. We cannot predict whether differences such as these might be characteristic of martian life.

(iii) Strategies for splitting the samples could be considered in the abstract, and this may provide some guidance to the sample collection operations of the proposed 2018 joint rover. However, a large part of this problem is sample-dependent-it matters what kinds of samples would be placed in the cache, and their specific character. Information at two levels of fidelity about the samples would be available for subdivision planning in two different time periods: (1) at the time the samples are selected, acquired, and cached, and (2) at the time of preliminary examination in the SRF. Sample subdivision strategies certainly should not be finalized before the latter, and these strategies should be reviewed and modified as testing proceeds in the SRF. The importance of real-time decision-making was also emphasized by Rummel et al. (2002). 
Table 10. Summary of Potential Rock Measurement Plan (Igneous and Sedimentary) Used to Estimate Optimum Sample Size

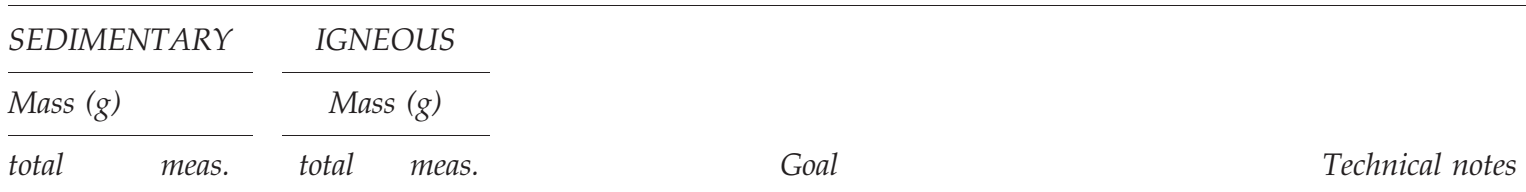

\begin{tabular}{|c|c|c|c|}
\hline \multicolumn{4}{|c|}{ Phase I. Initial Examination } \\
\hline 0.00 & 0.00 & $\begin{array}{l}\text { Get enough info to make decisions about } \\
\text { what to do with sample. How }\end{array}$ & $\begin{array}{l}\text { Preliminary examination using stand-off } \\
\text { instruments only; nondestructive }\end{array}$ \\
\hline 0.00 & 0.00 & $\begin{array}{l}\text { heterogeneous? How to subdivide? } \\
\text { Large-scale mineralogy and surface } \\
\text { organics }\end{array}$ & $\begin{array}{l}\text { Preliminary examination using stand-off } \\
\text { instruments only; minimally destructive }\end{array}$ \\
\hline
\end{tabular}

\section{Phase II. Planetary Protection}

1.50
Phase III. Research

1.85

1.21 Microanalysis of polished surfaces

Fluid inclusion analysis. Demountable thick sections (100 mm thick)

\begin{tabular}{|c|c|c|c|c|}
\hline 0.15 & 0.05 & 0.15 & 0.05 & $\begin{array}{l}\text { Microanalysis of individual subsamples - } \\
\text { number depends on heterogeneity }\end{array}$ \\
\hline 3.00 & 1.00 & $\begin{array}{l}3.00 \\
2.25\end{array}$ & $\begin{array}{l}1.00 \\
0.75\end{array}$ & Bulk analyses \\
\hline 1.50 & 0.50 & 1.50 & 0.50 & \\
\hline 0.30 & 0.10 & 0.30 & 0.10 & \\
\hline 0.60 & 0.20 & & & Clastic sediment component analysis \\
\hline
\end{tabular}

$\begin{array}{lcc}1.00 & 1.00 & \text { Follow-up for unexpected results } \\ \text { Phase IV. Sample Mass Held for Future Researchers } \\ 6.00 & 6.00 & \text { Future research } \\ 15.9 & 16.9 & \text { Subtotal } \\ 5 \% & 5 \% & \text { Factor for sample reuse and future } \\ & & \text { improvements in efficiency }\end{array}$

15.1

16.1
Total sample mass
Inorganic chemistry, organic chemistry, mineralogy, petrology, isotope geochemistry. Assume a need to prepare 5 thin sections and 1 thick section from each sample.
Inorganic chemistry, organic chemistry, mineralogy, petrology, isotope geochemistry
Soluble and insoluble organic analysis
Internal isochron geochronology, multiple isotopic systems
Bulk composition; stable isotope geochemistry
Gas extraction by crushing and heating to get major fluid phases $\left(\mathrm{CO}_{2}, \mathrm{H}_{2} \mathrm{O}\right.$, perhaps some noble gases)
Number of grains analyzed $(\geq 100)$ and number of distinct components (e.g., lithic, phosphate, plagioclase grains). Individual lithic grains of $\geq 1 \mathrm{mg}$ required for analysis

Pristine storage for future researchers

Current figure is a conservative guess. Needs detailed study by a future science planning team
We note that, if at least one of the life-related planetary protection tests proves positive, then obviously the priorities for how the sample mass would be used would change dramatically. This could be the most important scientific discovery of our lifetime! This position was clearly put forward by the Committee on Planetary and Lunar Exploration (COMPLEX, 2002).

As most recently articulated by Farmer et al. (NRC, 2009), detailed protocols for sample containment, handling, and testing, including criteria for release from containment, should be clearly articulated in advance of MSR. The protocols should be reviewed periodically as part of the ongoing oversight process in the SRF that would incorporate new laboratory findings and advances in analytical methods and containment technologies. International partners involved with the implementation of an MSR mission should be a party to all necessary consultations, deliberations, and reviews (NRC, 2009).
6.1.3. Scientific research. Phase III of the sample analysis workflow is the one in which the samples in the returned collection would be subsampled and allocated to scientific researchers for measurements with a variety of different analytical methods. In MEPAG E2E-iSAG (2011), we present an analysis of the kinds of measurements that would be needed to achieve the proposed scientific objectives of the MSR Campaign (Table 6), using instruments and sample preparation procedures available in 2011 and known to the authors of this report. As discussed above, analytic methodology will assuredly evolve between now and proposed receipt of samples, so the current analysis is a snapshot in time.

6.1.4. Requirement for replicate analyses. A fundamental principle of the scientific method is that measurements and other results need to be reproducible, including by different investigators and, if at all possible, by different methods. This is the primary means by which scientific 
discoveries are validated. Results in one lab that cannot be reproduced in another lab become suspicious. For example, disparate results may be due to error in one or both measurements or may indicate some unrecognized (possibly important) complexity in the samples. A second key principle, rooted in human nature, is that extraordinary discoveries require extraordinary evidence (attributed to Carl Sagan). In the case of MSR, we have high expectations for major discoveries (which is why the proposed MSR mission Campaign would be worth its cost), so it would be prudent to plan for enough sample material to demonstrate that the results are reproducible. Finally, the conventional "gold standard" for reproducibility in laboratory measurements is three independent determinations, both to ensure confidence in the results that agree and to provide some opportunity for evaluating any results that may differ between labs. The independence in such measurements is thus defined both by different scientific investigators and different laboratory facilities (and where appropriate, by different analytical methods). Discoveries obtained and validated by this process hold the greatest promise of being most widely accepted by the scientific community. The implication for the MSR Campaign is that the returned samples should be sized so that all critical measurements could be done in triplicate.

FINDING \#22: The samples should be sized so that all high-priority scientific measurements could be done in triplicate, in different laboratories, under the leadership of different principal investigators and, if possible, using different methods.

6.1.5. Reserve samples for future research. The preservation of material for posterity is an essential component of any curation policy. In this way, samples are kept for future generations of scientists to investigate, employing analytical techniques that have become more sensitive or have higher spatial resolution, and so on, than the methods available at the time samples were returned to Earth. Continued requests for Apollo material, over 40 years since it left the Moon, show the importance of this policy. A recent spectacular example of a new discovery made on an Apollo 16 sample is that by Borg et al., (2011).

- The Hayabusa team has specified that $45 \%$ of the sample be held in reserve (Zolensky, 2010, personal communication).

- Allocation of Apollo lunar rocks and soils is restricted to $50 \%$ of any specific sample. Allocation of additional material is possible only following very detailed (and skeptical) Curation and Analysis Planning Team for Extraterrestrial Materials (CAPTEM) review (Lofgren, 2010, personal communication).

- Current policy in Stardust is to hold $50 \%$ of the cometary sample in reserve (Zolensky, 2010, personal communication).

- For all meteorites, the long-standing rule used by the British Natural History Museum is no more than $1 \%$ of total holdings per request and no more than $10 \%$ in "curator's lifetime" (Grady, 2011, personal communication).

- In Dr. Penny Boston's work collecting and analyzing precious cave samples, she uses the rule that $33 \%$ of the sample needs to be held for future researchers.
In the case of MSR, the E2E team has mixed views regarding the fraction of sample material to hold in reserve, and this diversity of thought is likely also reflected in the community at large. It is unanimous that at least $25 \%$ of each sample should be set aside to support future science, and some team members feel this should be closer to $50 \%$. For the purpose of long-lead planning of sample mass, we have adopted the figure of $40 \%$.

FINDING \#23: Not less than $40 \%$ by mass of each sample should be set aside as a reserve to support future science.

\subsection{Implications for sample sizing: rock samples}

The size of individual samples is a key parameter of any sample return mission. Requirements for sample mass, volume, shape, and dimension feed directly into the design of the sampling system(s). Sample dimensions and quantity constrain the design of the cache and the attributes of the return flight system. Total sample mass, along with the mass of containing and supporting hardware, imposes stringent constraints on the MAV and the flight elements that would return the samples to Earth.

Numerous studies of MSR over the years have addressed the issue of sample mass, with varying degrees of scientific rigor. The most recent analysis, by the MEPAG ND-SAG (2008), concluded: "A full program of science investigations would likely require samples of $>8 \mathrm{~g}$ for bedrock, loose rocks and finer-grained regolith. To support required biohazard testing, each sample requires an additional $2 \mathrm{~g}$, leading to an optimal size of $10 \mathrm{~g}$." Although Table 4 of MEPAG ND-SAG (2008) did include the concept of a reserve sample, it did not explicitly account for replicate analyses, and the amount of sample mass needed to carry out certain high-value investigations was underestimated.

Rock sample sizing was estimated with the following assumptions, and summary results are shown in Table 10. The interested reader is referred to MEPAG E2E-iSAG (2011) for more details. Because returned sedimentary and igneous rocks would be used differently to achieve the proposed scientific objectives, the investigations and sample mass/investigation are presented separately in Table 10 for these two rock types. This analysis shows that sample sizing of 15$16 \mathrm{~g} /$ sample is optimal.

- All required analyses may be done on any sample.

- All samples would be tested for evidence of extant life and biohazard (planetary protection).

- All high-priority analyses would be performed in triplicate.

- A portion of every sample would be retained for follow-up analysis in the event of unexpected results.

- A portion of every sample would be retained for future research.

- Sample reuse and future improvements in efficiency would reduce the demand for sample material.

FINDING \#24: The recommended mass/sample for rock samples is $15-16 \mathrm{~g}$. The needs for sedimentary and igneous rocks are slightly different. 
FINDING \#25: There would be significant scientific consequences to returning a sample that is significantly undersized (determination of the threshold size would require additional discussion by a future science planning team). An important science priority is to be able to recognize such cases early enough on Mars that faulty sample collection attempts could be rejected and the samples reacquired.

\subsection{Implications for sample sizing: regolith samples}

For each regolith sample, investigations in each of the following areas are thought to be necessary to achieve the scientific objectives proposed (key input acknowledged from Mike Hecht and the Granular Materials Focus Group; see also MEPAG E2E-iSAG, 2011, Section 3).

- Physical properties (Shape, texture; Size distribution; Diffusivity, tortuosity, permeability; Surface area and porosity)

- Chemistry (Soluble ions; pH, Eh, trace metals, etc.; Volatiles and organics; Surface analysis)

- Mineralogy (Distributions; Crystal structure; Elemental composition; Magnetic properties)

- Origins (Age; Stable isotopes)

- Spectroscopy (Color; Raman)

- Biology (Viable microbes; Dead microbes; Biomarkers (amino acids); Organic/inorganic carbon)

- Human safety (Toxicity-chemical; Toxicity-inhalation; Electrostatics; Adhesion)

While there has been great progress in analysis techniques for minute samples, grain diversity and handling considerations suggest that a minimum of $5-25 \mathrm{mg}$ would be necessary for each investigation described above except the search for viable microbes, which would require at least $300 \mathrm{mg}$ per sample. As discussed above in Section 6.1.4, it is a high priority that the samples be large enough to support three redundant measurements for each investigation. The amount of regolith sample that would be required to support planetary protection testing is hard to estimate but should not be assumed to be less than $10 \%$, as per arguments discussed above in Section 6.1.2. Maintaining soil samples in a pristine state during transport and analysis by different laboratories would be more challenging than for rock and gas samples, and greater loss or compromise of samples is expected. Accordingly, for regolith samples, it is recommended that $67 \%$ of the sample be retained for future analysis.

Adding up all the above factors leads to a minimum recommended sample size of about $7.5 \mathrm{~g}$. Some reduction of this total may be possible by using a carefully structured program of sample reuse (Finding \#26). Granular materials have lower density than rocks, particularly after they have been disturbed. For example, the average density of the lunar regolith is $1.5 \mathrm{~g} / \mathrm{cm}^{3}$ in the top $15 \mathrm{~cm}$ (Heiken et al., 1991). The bulk fines delivered to the $X$-ray fluorescence spectroscopy instrument on Viking had a density of $1.10 \pm 0.15 \mathrm{~g} / \mathrm{cm}^{3}$ (Clark et al., 1977). Dust is deposited on the surface with an estimated bulk density of $0.95 \mathrm{~g} / \mathrm{cm}^{3}$ (Moore, 1991). Assuming a density of $1.15 \mathrm{~g} / \mathrm{cm}^{3}$ for disturbed soil, a $7.5 \mathrm{~g}$ sample would need a sample tube with a volume of at least $6.5 \mathrm{~cm}^{3}$. If collecting samples of this size were overly difficult, in the judgment of the E2E-iSAG, useful science could still be achieved with samples as small as $1 \mathrm{~cm}^{3}$ (by reducing the archived fraction to $40 \%$, reducing redundancy to 2 , using the minimum quantity for each measurement, etc.), but this is not recommended.

FINDING \#26: A relatively full program of scientific analysis could be done on a regolith sample of about $7.5 \mathrm{~g}$ (which would have a volume of about $6.5 \mathrm{~cm}^{3}$ ).

\subsection{Implications for sample sizing: atmospheric gas samples}

A detailed analysis of how the gas research community would process a returned martian gas sample is presented in Section 4 of MEPAG E2E-iSAG (2011). The minimum quantity of gas needed to achieve all high-priority objectives would be $1.9 \times 10^{-5} \mathrm{~mol}$, which is equivalent to $\sim 50 \mathrm{~cm}^{3}$ at Mars ambient temperature and pressure (see Section 4 of MEPAG E2E-iSAG, 2011). This calculation is based on the following assumptions:

- Fifty percent of the returned gas would be saved for the future.

- Then-current allocations would be made to three laboratories, each of a size necessary to make three determinations. Thus, each aliquot would be one-ninth of $50 \%$ of the original sample.

- The mass spectrometer is of the type used in the ETH lab in Zurich, which is capable of measuring all the noble gases in a single run. Note, however, that a mass spectrometer dedicated to Xe only [e.g., RELAX, J. Gilmour, University of Manchester (Gilmour et al., 1994)] would require a lower gas quantity for a precise analysis of just the Xe isotopes. Also, new multicollection noble gas mass spectrometers under development may allow Xe analysis with a gas amount lower than that presented here (Alex Meshik, personal communication, 2011).

- The least-abundant high-priority components in a martian atmospheric gas sample, which drive the minimum sizing calculations, would be ${ }^{124} \mathrm{Xe}$ and ${ }^{126} \mathrm{Xe}$. For ${ }^{124} \mathrm{Xe}$, a quantity of $2.4 \times 10^{-17} \mathrm{~mol}$ of ${ }^{124} \mathrm{Xe}$ is required to generate a signal with a statistical counting error of less than 1\% (ETH noble gas mass spectrometer).

- The measured Ar fraction in the martian atmosphere is $1.6 \%$ (Viking). The ${ }^{36} \mathrm{Ar} /{ }^{132} \mathrm{Xe}$ is thought to be between 350 and 900 (Viking; Pepin, 1991; Bogard and Garrison, 1998). For these calculations, we used the most conservative estimate of 900 . The ${ }^{124} \mathrm{Xe} /{ }^{132} \mathrm{Xe}$ is currently believed to be 0.0038 (meteorite data, Swindle et al., 1986; Garrison and Bogard, 1998). In summary, the fraction of $\mathrm{Xe}$ in the martian atmosphere is $\sim 3 \mathrm{E}-6 \%$ (32 ppb) and the fraction of ${ }^{124} \mathrm{Xe}$ of total $\mathrm{Xe}$ is $\sim 0.07 \%$. Using these assumptions, $2.4 \times 10^{-17} \mathrm{~mol}$ of ${ }^{124} \mathrm{Xe}$ would be contained in an atmospheric sample of $1.9 \times 10^{-5} \mathrm{~mol}$.

- Using $P V=n R T$, with $P=700 \mathrm{~Pa}$ and $T=223 \mathrm{~K}$, we arrive at a calculated volume of $50 \mathrm{~cm}^{3}$.

If it were not possible to return enough gas to measure ${ }^{124} \mathrm{Xe}$ and ${ }^{126} \mathrm{Xe}$, the next least-abundant species of high scientific interest would be ${ }^{128} \mathrm{Xe}$ and ${ }^{78} \mathrm{Kr}$. The minimum sample size needed for those determinations (as well as all species more abundant than this), with the same assumptions as above, would be about $1.0 \mathrm{~cm}^{3}$ at Mars ambient 
temperature and pressure, which is a factor of 50 reduction from the above. However, ${ }^{124} \mathrm{Xe}$ and ${ }^{126} \mathrm{Xe}$ are important for the following reasons. Xenon is one of the most important elements for interpreting martian atmospheric formation and evolution. Its nine isotopes would allow us to unravel the starting composition of the atmosphere and processes that later altered its composition. The importance of ${ }^{124} \mathrm{Xe}$ and ${ }^{126} \mathrm{Xe}$ is as follows: (i) They are the rarest isotopes and, therefore, have been measured in meteorites with the largest uncertainties. (ii) In meteorites, there are large cosmogenic effects (up to $90 \%$ ) because of their time spent in space, further increasing the uncertainties. (iii) Many other Xe isotopes have other sources: for example, ${ }^{129} \mathrm{I} \rightarrow{ }^{129} \mathrm{Xe},{ }^{244} \mathrm{Pu}$ fission $\rightarrow{ }^{131-136} \mathrm{Xe}$ (see review by Swindle, 2002). ${ }^{124,126} \mathrm{Xe}$ have no other contribution (apart from cosmogenic, which however should be negligible in the atmosphere) and would therefore be valuable to reveal the extent of mass fractionation of the starting composition and thus the degassing history (Pepin, 2006).

The development of multicollection mass spectrometers for noble gas analysis should reduce the required gas amount for a precise Xe analysis in the future. Note also that making gas sample allocations to only three laboratories would be a minimum credible plan-returning a substantially larger sample than this would be greatly welcomed by the science community. If a simple compressor were included, this quantity of gas could be packaged in a volume of $5 \mathrm{~cm}^{3}$ at a pressure of $10 \times$ Mars ambient or a volume of $0.5 \mathrm{~cm}^{3}$ at a pressure of $100 \times$ Mars ambient.

FINDING \#27: We find that returned martian atmospheric gas samples should have a size of at least $1.9 \times 10^{-5}$ mol, which is equivalent to $\sim 50 \mathrm{~cm}^{3}$ at Mars ambient temperature and pressure, in order to support a full range of high-priority scientific investigations.

\section{Capabilities on Mars Needed to Select, Acquire, and Preserve the Samples}

To establish the field context described in Section 3 of this report, to recognize the kinds of samples described in Section 2 and 4 of this report, and to access and acquire them at the kinds of sites described in Section 5 of this report, certain field capabilities are implied. These capabilities are described in the following sections. In particular, certain kinds of instruments and a sampling and packing system must be present. Some attention must be given to the preservation of the scientific value of the samples in the time interval between when they were acquired and when they are analyzed (which could be several martian years).

\subsection{Observations required to understand geological context}

As described in Section 3.2, to obtain the martian sample suites most suitable for further analysis on Earth, it would be necessary to make a wide range of field observations. These field observations would be essential to guide the sampling process, and they are critically important when it comes to interpreting the results of sample analyses on Earth. For example, in a sedimentary sequence on Earth, a field geologist typically acquires an overview of the area by examining rock types, textural features (e.g., grain size and sorting), bedding characteristics (contacts, lateral and vertical variations in thickness, extent geometry), stratigraphic relationships, and so forth in order to interpret the local geology. On this basis, a sampling strategy could be devised to answer particular problems. Such observations are essential to understanding what a sample represents. Field geology on Mars would be no different, as demonstrated by our experience with the MER mission (e.g., Squyres et al., 2004a; Grotzinger et al., 2005; McLennan et al., 2005). Accordingly, it would be necessary to analyze the geological setting at the landing site in order to identify where to sample and to establish the geological context of the samples to maximize their science value.

Accordingly, to successfully acquire the most important available samples within a reasonably well-defined geological context, and to acquire the necessary contextual information to enable the highest-priority returned sample science objectives to be met through future sample analyses, the following field capabilities would be required:

(1) Ability to detect and correlate variations in mineralogy, chemical composition, textures/structures (at micro-, meso-, and macroscale) in outcrops;

(2) Capability to make a sufficient number of interrogations, by the onboard instruments, of the outcrops to adequately understand the geological context;

(3) Ability to "see" the rocks below their coverings of dust and surface weathering products;

(4) Mobility range and lifetime sufficient to conduct exploration outside the landing ellipse.

Organic geochemistry. In addition to the measurements listed above, we conclude that the capability to detect organic material is highly desired. This reaffirms previous findings. The ND-SAG (2008; Sections IV-E, IV-F) indicated that the data would be of high scientific priority for sample selection but also said that color imagery, remote spectroscopic observations, and contact geochemical/mineralogical analyses constitute the minimum set of techniques that would be needed to optimize sample selection, which thus indicates that organic detection would not be mandatory (i.e., "required"). The MEPAG MRR-SAG (2010) presented a mission vision based on the premise that the information to make effective sample selection decisions could be made only by using fast, relatively light, arm-mounted instruments. They recommended a suite of measurement capabilities (see their Section 6), including organic geochemistry, but without distinguishing "required" from "desired." The MEPAG MRR-SAG (2010) study flowed into the NRC (2011) concept for MAX-C, which included a potential deep-UV instrument (for detecting organics), but this was a singlepoint design not based on requirements.

Samples that may contain organic material would be of high interest to Objective A1. Thus, information about the presence or absence of organic matter would be valuable to the sample selection process. However, since there are many other reasons to justify the return of samples, and we do not know whether martian samples accessible to our sampling systems would contain organics in a concentration or chemical form that is detectable by in situ instruments, establishing the detection of organics as a prerequisite to caching a sample is both unjustified and unwise. By analogy with hydrocarbon "source rock" studies on Earth, collecting 
materials that have a high likelihood of preserving organic compounds or any kinds of potential biosignatures is a viable strategy. Examples of such rocks include carbonate, phyllosilicate, or silica-rich materials. Color and textural features, such as laminae or stromatolite-like layers, would also inform recognition.

If organic detection equipment was included in the mission, then it should represent an analytical step intermediate between in situ visual inspection and ex situ comprehensive analysis. Such a step would inevitably reduce risk in successful target rock selection for astrobiological purposes. Yet, as an intermediate stage of analysis, the lack of conclusive organic matter detection by an in situ instrument on an apparently otherwise suitable rock would not preclude sample selection, given that more exacting tests would await back on Earth.

7.1.1. Scale of required field observations. To derive the greatest scientific benefit from returned samples, it would be necessary to acquire sufficient relevant contextual information while on the martian surface so that a complete picture could be built up of the geological history of the materials. Contextual information must be integrated across multiple scales: macroscopic (e.g., regional scale, such as observable from orbit or across multiple outcrops examined by the rover), mesoscopic (e.g., outcrop-scale features such as bedding, larger clasts, basalt pillows, veins) and microscopic (e.g., sand grains and mineral crystals, laminations, voids, veinlets). Observations and measurements of visible and compositional features would need to be correlated across these different scales.

On the macroscopic scale, collection and synthesis of the types of orbital imagery and spectral data would be needed to define regions of interest from which the final MSR landing site would be chosen. The analysis of orbital imagery is an ongoing process that, for example, enabled definition of the MSL landing site (e.g., Rogers and Bandfield, 2009; Golombek et al., 2011). Such datasets would provide the overall morphological and mineralogical information required to select the most appropriate landing site to meet the science objectives. Orbital data would also enable planning of general and specific operational sequences that would produce the most valuable science outcomes and the most effective mission operations. The datasets would also need to be correlated and compared with observations made in situ during the mission to aid interpretation of the local geology. This latter consideration highlights the need to be able to make correlations between measurements (such as mineralogy) acquired from orbit with measurements made in situ.

Mesoscopic-scale observations are remote measurements made by instruments located on the rover and, as for the macroscopic-scale observations, are needed to provide an integrated set of morphological and mineralogical information. In this case, however, the observations would be at outcrop level with the intent to survey and characterize features in the vicinity of the rover. Initial imagery acquired by a mast-mounted camera would be aimed at identification of general areas where further examination should be carried out. Examples of questions to be addressed include: Is the outcrop layered? What are the extent, geometry, and distribution of the layers? Do they differ in internal structure, texture, and the nature of contacts with surrounding units? Are additional features, such as pillow lavas, chilled lava flow margins, pyroclastic bombs, concretions, clasts, crossbedding, or veins present? Is there any indication of size sorting or crystal settling? And so on. In addition to imagery, compositional analyses (e.g., mast-mounted spectroscopy) would need to be acquired and correlated with visual imagery to constrain the nature and significance of features seen in the images. These combined measurements would be needed to enable efficient, effective targeting of the most important features in the vicinity of the rover for more detailed analysis and potential sampling. The stratigraphic investigation of the Burns Formation at Meridiani Planum (Grotzinger et al., 2005, 2006) and pyroclastics at Home Plate in Gusev Crater (Squyres et al., 2007; Lewis et al., 2008b) provide good guidance for such approaches on Mars.

Once a specific outcrop (or section within an outcrop) has been identified for close-up examination or potential sample collection, a set of measurements would need to be made at the outcrop surfaces. These measurements would be needed to determine the physical appearance, arrangement, and composition of centimeter- to submillimeter-scale structures and textures. While a few measurements may be carried out on undisturbed (weathered or dust-covered surfaces), in most cases it would be necessary to remove any adhered dust and surface weathering rinds or features before analyses are carried out, so that fresh, unaltered material could be examined. Investigation of rock surfaces at the microscale would be needed to allow direct, in-context evaluation of small-scale structures, fabric, and texture, including the size, shape, and heterogeneity of sedimentary grains or igneous crystals; the presence or otherwise of voids or concretions; porosity; cementation; vesicles in lavas (and any associated infills); the presence or otherwise of clasts (and their shape and size distribution); the existence of flow structures or sedimentary structures, and so on.

Along with imagery, compositional data (i.e., chemistry, mineralogy) would need to be acquired in such a way that the composition of the different features observed could be distinguished. Investigation of the Burns Formation with the MER turret instruments provides an example of small-scale analysis (e.g., McLennan et al., 2005). However, the MER experience also highlights the need for additional capability so that the composition of microscale features (e.g., grains, laminae, concretions) visible in close-up images could be differentiated. In the MER case, the inability to confidently identify non-iron-bearing minerals and to correlate microtextural features with mineralogical and chemical information was a significant shortcoming in fully interpreting the data (MEPAG MRR-SAG, 2010). To meet the high-priority science objectives of the proposed MSR Campaign, it would be essential to have access to this kind of contextual information in situ in order to select the samples, as well as to support the interpretation of future sample analyses.

All three sets of measurements-macroscopic, mesoscopic, and microscopic-should be integrated so that fine-scale features could be understood within their larger-scale context and large scale observations could be ground truthed with higher-resolution measurements. Once samples are returned to Earth, the wealth of contextual information so acquired would be essential for confident interpretation of the evolutionary history of the site and samples. Contextual detail would also enable conclusions drawn from measurements made at a local site (single sample or suite of samples) 
Table 11. The Pasteur Payload

\begin{tabular}{|c|c|c|}
\hline \multicolumn{3}{|c|}{ CURRENTLY APPROVED PASTEUR PAYLOAD } \\
\hline INSTRUMENT NAME & DESCRIPTION & COMMENT \\
\hline PanCam (WAC+HRC) & Panoramic camera system & Mast-mounted \\
\hline MOMA & $\begin{array}{l}\text { LD-MS + Pyr GC-MS organic molecule } \\
\text { characterization }\end{array}$ & \\
\hline MicrOmega IR & IR imaging spectrometer & Analytical Laboratory Drawer (ALD) \\
\hline Mars-XRD & X-ray diffractometer + X-ray fluorescence & instruments: Rover-body, internal \\
\hline Raman & Raman spectrometer & \\
\hline Life Marker Chip & Biomarker detection, immunoassay & \\
\hline CLUPI & Close-up imager & Drill-mounted \\
\hline WISDOM & Shallow ground-penetrating radar & $\begin{array}{l}\text { Rover-body, internal electronics, } \\
\text { external antennas }\end{array}$ \\
\hline Ma_MISS included in $2.0 \mathrm{~m}$ drill & IR borehole spectrometer & Drill-mounted \\
\hline
\end{tabular}

to be extrapolated to regional scale (relationship between different outcrops and deposits) and, when integrated with results from orbiting instruments, allow global-scale interpretation of geological features.

FINDING \#28: Integration of visual and compositional observations from macroscopic (outcrop, regional) scales down to microscopic (submillimeter) scales would be essential for robust geological interpretation in support of sample selection and provision of context for sample analyses on Earth.

\subsection{The Pasteur payload}

As of this writing, ESA and NASA are pursuing a formal collaboration for the exploration of Mars. This collaboration is focusing initially on the 2016 and 2018 mission opportunities. However, it is anticipated that the collaboration would continue across several launch opportunities through to the return of samples from Mars and even beyond. The charter of the E2E-iSAG indeed initially was predicated on the assumption that two rovers (MAX-C and ExoMars) would be delivered together to the same landing site in 2018.

In response to budgetary constraints and other ESA/ NASA considerations, the E2E-iSAG was asked in May 2011 to consider additional assumptions about the proposed 2018 rover in making our recommendations, which bear most directly on the nature of instrumentation that such a rover would need to support the MSR Campaign. These assumptions are summarized as follows:

(1) The mission would consist of a single joint rover to be delivered by the MSL skycrane system. (For the purpose of this report, we refer to this as the 2018 joint rover.)

(2) The mission would support both returned sample science (based on science priorities updated via the E2E-iSAG analysis) and in situ science derived from previously defined ExoMars priorities.

(3) The rover would include the ExoMars Pasteur payload that was previously selected. Whether additional instruments for sample selection/caching are required is to be analyzed by $\mathrm{E} 2 \mathrm{E}$, and also assume that selection of additional instruments would be via some future joint announcement of opportunity.
Accordingly, the discussion below incorporates these added assumptions/requests to the degree possible that is also consistent with the original charter of the E2E-iSAG.

For reference, the currently planned instrumentation of the current ExoMars Pasteur payload is summarized in Table 11. The science payload would consist of a mast-mounted panoramic camera system (PanCam), a body-mounted groundpenetrating radar system, a ( $2 \mathrm{~m}$ deep) drill-mounted microscopic imager and IR spectrometer, and an Analytical Laboratory Drawer (ALD) consisting of five instruments capable of mineralogical, geochemical, and organic geochemistry measurements. In its configuration as of May 2011, the Pasteur payload did not include any robotic armmounted instrumentation.

7.2.1. Potential use of the instruments of the Pasteur payload to support the objectives of the MSR Campaign. The instruments of the Pasteur payload (Table 11) have significant potential to produce information relevant to the proposed MSR Campaign. They could generate data on mineralogy, geochemistry, and visual features that would be relevant to the observational needs described in Section 4 of this report:

(1) Mars-XRD and MicrOmega IR. These two instruments have a potentially powerful ability to interpret mineralogy.

(2) CLUPI. A close-up imager of some sort is considered mandatory for geological interpretation. However, the positioning of CLUPI on the body of the rover (in the designs as of Aug. 2011) is far from ideal-this kind of instrument would be far more valuable when positioned on a robotic arm.

(3) Panoramic camera system. A sampling rover cannot be operated without this kind of instrument.

(4) The Raman spectrometer would rapidly detect a range of organic functional groups in addition to their mineralogical host materials (e.g., Jehlička et al., 2009). Functional group analyses could indicate the presence and preservation state of organic matter, and certain collected responses could imply the existence of specific organic entities such as organic pigments.

(5) The Mars Organic Molecule Analyser (MOMA) could operate as a laser desorption mass spectrometer to 
study large macromolecules and inorganic minerals, and a gas chromatograph-mass spectrometer for the analysis of volatile and semivolatile organic molecules (e.g., Becker et al., 2010). Gas chromatograph-mass spectrometry readily recognizes the fossil remains of life. Stepwise heating of samples in the presence of a derivatization agent could prepare polar organic molecules characteristic of recent or exceptionally wellpreserved life, such as amino acids, for analysis.

(6) The Life Marker Chip (LMC) instrument would utilize biotechnology measurement techniques to detect organic compounds that reflect past or present life (e.g., Parnell et al., 2007). The instrument would utilize the recognition and binding properties of protein-based receptor molecules labeled with a fluorescent dye to signal successful compound detection.

The Raman spectrometer, MOMA, and LMC are particularly relevant to Objective A1.

However, while the Pasteur instruments are of significant potential relevance to the MSR Campaign, there are several factors that limit the ability of the instruments to perform the kinds of required in situ observations described in Section 7.1. One potential issue is the limited ability to deliver sample material to the instruments in the ALD. Baseline planning as of August 2011 was that the only sample transfer pathway would be from the deep drill to the ALD. The addition of a capability to transfer samples from the armmounted corer to instruments in the ALD would be of significant scientific value. This capability could greatly amplify the contribution of these instruments to sample selection decision making. An important caveat, however, is that the time required to acquire samples and carry out measurements in the ALD would restrict the number of times such a capability could be used for characterizing the local geology and selecting samples. A further limitation on the utility of the ALD instruments is that rock samples would be crushed prior to analysis. Thus, spatial information at a scale smaller than the samples would be lost, as would the possibility of determining the relationships between visible and compositional features identified by the instruments. As described in Section 7.1, this spatially correlated information is crucial for understanding the origin of the measured features and their significance within the larger context. Because of these limitations, several additional measurement capabilities would be needed to achieve the proposed MSR science objectives.

\subsection{Measurement needs of the proposed 2018 joint rover in addition to those of Pasteur}

7.3.1. Mast-mounted instruments. The range of scientific objectives of the MSR Campaign would require variation in sampled materials and thus require a landing site that is geologically diverse. To maximize productivity and efficiency of operations while the rover is exploring a geologically diverse terrain, the instruments must be able to quickly acquire information about the local geology and survey possible sampling areas. Combining high-resolution colorstereo imagery with mineralogical information about outcrops, rocks, and soils would best accomplish the job of understanding a diverse geological environment and quickly prioritizing candidate sampling targets. This implies the need for remote imaging and mineralogy capabilities via mast-mounted instruments on the rover.

The MER missions demonstrated the value and importance of rover-based remote sensing instruments. The highresolution multispectral stereo imager known as Pancam far exceeded the grayscale imaging capability of the rovers' engineering cameras, which allowed for rapid assessment of the morphology and composition of the outcrops, rocks, and soils at both landing sites (e.g., Bell et al., 2004a, 2004b). Working in concert with Pancam, the IR spectrometer known as Mini-TES provided detailed information on the mineralogy of these materials without the need for contact with them (e.g., Christensen et al., 2004a, 2004b). Together, the data from both instruments were used routinely to direct the rovers to targets of interest for subsequent measurements from the arm-mounted instruments and to place these measurements into a geological framework. The operational efficiency of employing remote sensing instruments for reconnaissance significantly enhanced the productivity of both rovers in evaluating the geological environments they encountered (e.g., Squyres et al., 2004b, 2004c). The proposed 2018 joint rover would derive similar benefits if the rover were equipped with comparable remote sensing capabilities.

7.3.2. Arm-mounted instruments. Measurements achievable only through contact by instruments mounted on a rover arm would be a critical part of the winnowing process from many hundreds of observations by the mast-mounted remote sensing instruments to a limited number of samples suitable for return to Earth. Sample coring and caching would be both time-consuming and potentially risky, and should therefore be undertaken only on carefully chosen rocks/soils. The arm-mounted instruments would be critical for providing detailed information sufficient to decide where to sample.

Some of the features of greatest interest in guiding sample selection occur at scales of centimeters to submillimeter (equivalent to that of component mineral grains, laminae, veins, etc.). Imaging at this scale provided important discoveries by MER with use of the arm-mounted Microscopic Imager (e.g., Herkenhoff et al., 2004). Such capability would be required for the MSR Campaign to help guide sample coring placement, characterize microtextures in the immediate vicinity of the sample site, and assist in recording the orientation of samples. The MERs also were equipped for chemical (Alpha Particle X-Ray Spectrometer) and mineralogical (Mössbauer spectrometer) measurements via arm-mounted instruments. These general types of measurement capabilities (though not necessarily these instruments), which proved to be critical in providing a more complete picture of the geological materials and their environments of formation and alteration (e.g., Gellert et al., 2004; Klingelhöfer et al., 2004), are also considered requirements for the MSR Campaign. Although the two MER instruments measured at the centimeter scale, we propose that the overall scientific value of the selected samples would depend on the integration of information about both visible features (textures, structures) and composition at millimeter to submillimeter scales.

Among the geochemical measurements that could be performed by an arm-mounted instrument, the ability to characterize organic content is also highly desirable. However, the limited detection limits and likely limits on the ability to 
characterize any detected organic compounds resulted in a conclusion that, while such measurements would add significantly to the likelihood of obtaining the best available samples, such a capability is highly desirable but not required.

The success of the MERs' arm-mounted instrument observations was due in no small part to the ability to remove surface dust and weathered surfaces on rocks with the armmounted Rock Abrasion Tool (RAT; Gorevan et al., 2003). A comparable capability is viewed as necessary for the proposed 2018 joint rover, given that it would enable much better decision-making by way of the superior context imaging in that mineralogical and chemical measurements could be made on clean and less-weathered rock surfaces. Future studies will need to evaluate things like topography of the sampling surface (both before and after use of the surface preparation device), arm reach, outcrop angles/ slopes for example, and other features to be sampled. It will be key to distinguish the needed capabilities of the arm/ coring device.

7.3.3. Summary of on-Mars measurement needs in excess of Pasteur. Although the Pasteur payload has the capabilities of meeting some of the measurement requirements of the MSR Campaign, the inability to measure and correlate visual and compositional characteristics in context on the surface of outcrops (both remotely with mast instruments and up close with arm instruments) necessitates the requirements for additional instrumentation. These can be summarized as follows:

(1) In addition to the Pasteur PanCam imager, a remote sensing instrument capable of detecting mineralogy would also need to be present on the mast.

(2) A robotic arm would be needed that could carry instrumentation capable of (a) microscopic imaging at submillimeter resolution; (b) mineralogical detection, preferably with submillimeter resolution; (c) determination of chemical composition; and (d) removal of adhered dust and weathering products. Capability for detection of organic molecules is highly desired and would add considerable scientific value.

(3) The ability to use the ALD to analyze samples collected by the robotic arm would also be highly desirable, particularly for detection and analysis of organic matter and for determining mineralogy.

FINDING \#29: Mast, arm, and onboard lab instruments would all be of value for achieving the science objectives of the MSR Campaign. However, each would play a different tactical role in sample selection and establishment of geological context.

FINDING \#30: In order to recognize the geological characteristics of interest and to provide a proper basis for sample selections, two measurement types would be required from the mast and 3-4 more from the arm. Onboard laboratory measurements such as provided by the ALD would be highly desirable for the purpose of sample selection and establishment of geological context.

FINDING \#31: The value of onboard lab instruments would be greatly increased if samples could be passed from the arm corer to the ALD.

\subsection{Sample collection and preservation system}

Integrated concepts for core sample acquisition and caching consistent with a potential application to the mission objectives described in this report have been developed and either published (e.g., Collins, 2009; Backes et al., 2010, 2011) or attributable to private corporations in the public domain (e.g., ASI, Honeybee, Swales). The concept would utilize a 5degree-of-freedom arm to deploy and manipulate a rotary percussive coring tool. The coring tool itself would provide coring, core break-off, core retention, as well as bit capture and release for bit change-out. A sample would be acquired directly into a single-use sample tube within the coring bit, and bit change-out would be used to transfer the sample tube to the caching subsystem where it would be sealed and stored. The sample storage canister, containing the individual sample tubes, could be left on the rover or deposited on the surface for later pickup by a subsequent mission.

As discussed above in Section 2 of this report, it is important that the sample acquisition system have the ability to drill into loose rocks, in addition to outcrop. This raises follow-up questions that will need to be considered by a successor panel, such as how small the rocks could be to avoid their moving when force is applied. In addition, the ability to deploy the corer to a wide range of targets away from the rover body, and against rover hazards, would be a key capability.

This particular caching subsystem concept is referred to as the Sample Handling, Encapsulation, and Containerization (SHEC) subsystem. Bit change-out and sample caching are combined in the design. There is one opening in the SHEC subsystem design; the coring tool interface port for transferring a coring tool bit. The tool bits are stored in bit holders on the bit carousel. A 2-degree-of-freedom transfer arm internally transfers sample tubes between bits on the bit carousel, plugs on the sample carousel, plugging station, and tube chambers in the sample canister. This system has the ability to switch $25 \%$ of the samples if higher-priority samples are found later in the mission (Section 3.3). The sample canister is in the center of the sample carousel and could be removed from the top of the SHEC by the rover arm; this would enable either deposition on the surface for later pickup by a rover from a possible future mission or direct removal by that future rover.

7.4.1 Sizing the sample cache. Previous studies of MSR missions have mostly assumed a returned sample mass of about $500 \mathrm{~g}$ based on trades of sample mass against the MAV and MSR-O projected capabilities and costs (e.g., Price et al., 2000). In this study, we estimated the minimum sample mass to be returned by examining how large each sample should be to do the desired analyses and how many different samples would be needed to sufficiently exploit the scientific potential of a particular landing site. The relationship between mass/sample and number of samples, and how these two factors contribute to the overall size of the returned sample collection, is shown in Fig. 7.

For rock samples, the optimal mass/sample (derived in Section 6 of this report) is $15-16 \mathrm{~g} /$ sample, with minor differences between sedimentary and igneous rocks. A comfortable number of rock samples, as discussed in Section 6 of this report, is about 30-35. The product of these two figures 

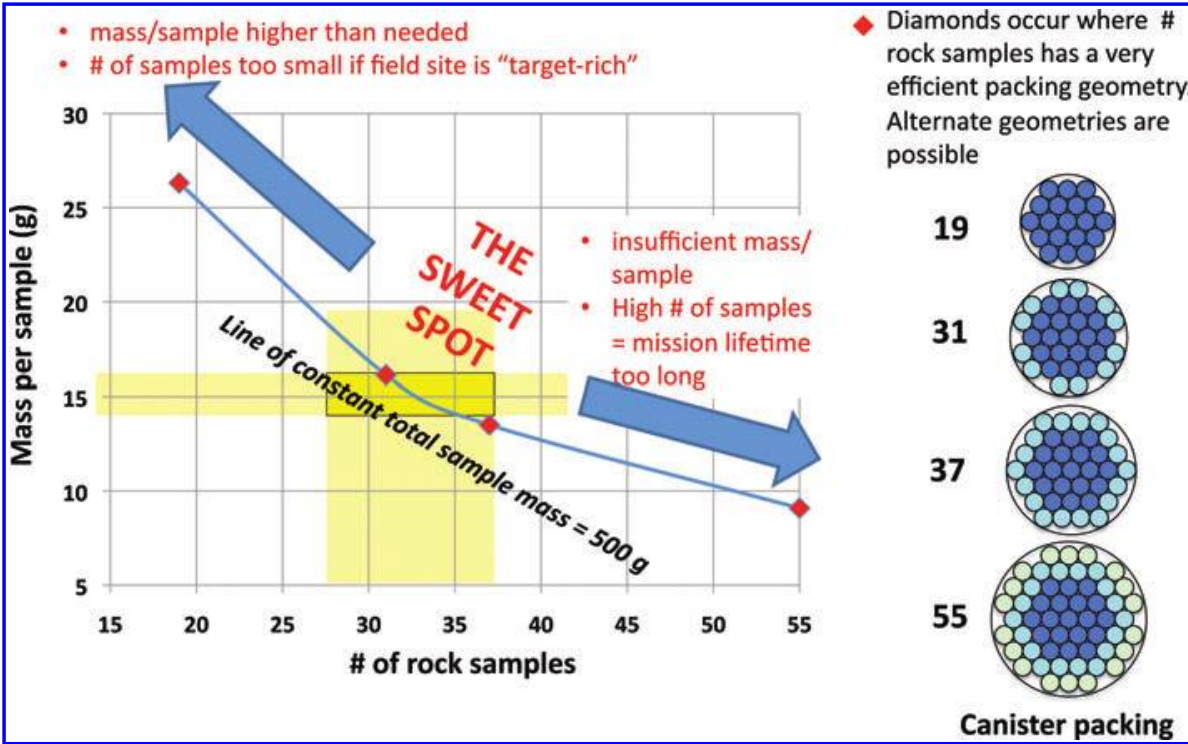

FIG. 7. Relationship between sample size and sample numbers, including several possible packing scenarios. Color images available online at www.liebertonline.com/ ast

is close to $500 \mathrm{~g}$. If smaller numbers of larger samples were collected, there would be a significant risk that too few samples would be collected to characterize the landing site. On the other hand, if larger numbers of smaller samples were collected, there may be insufficient mass to carry out the analyses on Earth, and the amount of time needed to collect the samples may add inordinate amounts of time to required mission life.

The density of the shergottite and nahklite meteorites (martian igneous rocks for which we have data) averages 3.01 and $3.11 \mathrm{~g} / \mathrm{cm}^{3}( \pm \sim 7 \%)$, respectively (Britt and Consolmagno, 2003; Coulson et al., 2007; Macke et al., 2011). The bulk density of the sedimentary rocks on Mars has not been measured, but a starting place for discussion is $2.25-2.5 \mathrm{~g} /$ $\mathrm{cm}^{3}$ [estimated by using the mineralogies reported in McLennan and Grotzinger (2008) and Ruff et al. (2006) and the porosities reported in Perl et al. (2007)—Budney, written communication, 2011]. For reasons of both porosity and grain density, however, samples with density higher or lower than this could be encountered. While noting these uncertainties, the sample masses described above, if these densities are used, would be equivalent to volumes of $\sim 5.3$ and $\sim 6.7 \mathrm{~cm}^{3}$ for igneous and sedimentary rocks, respectively. Note that in a practical sense it is impossible to pack a rock sample into a sample tube with $100 \%$ efficiency. Thus, the sample tubes would have to be larger than the above volumes in order to achieve the amount of sample mass required. The decision on sample tube sizing needs to be guided by scientific considerations, experimentation in a sampling test bed, and margin planning considerations (see MEPAG E2E-iSAG, 2011).

For regolith samples, a minimum volume of approximately $6 \mathrm{~cm}^{3}$ for each regolith sample would be required (Section 6 of this report). Depending on porosity, this is approximately equivalent to $\sim 8 \mathrm{~g}$, although with high uncertainties.

An important conclusion is that the sample size for regolith and rock samples would be approximately equal in a volume sense. This means that it may be possible to use the same sample tubes for both types of samples. Using a stan- dard sample tube size would have important benefits to engineering.

FINDING \#32: The collection should be sized to a total sample mass of about $500 \mathrm{~g}$.

7.4.2. Sample packing. Since volume would be at a premium in the flight elements of the MSR Campaign, it would be necessary to pack the samples in the most spatially efficient manner possible. If the rock and regolith samples are collected into cylindrical sample tubes of a standard size, these tubes could be packed into a cylindrical canister by using one of several possible close-packing geometries. Although the diameter versus length combination for the individual sample tubes has yet to be worked out, the closepacking geometry would lead to a limited number of efficient solutions-the most relevant options involve 19, 31, 37, and 55 slots (see Fig. 7). It would be possible to store the atmospheric sample in one of the slots in such a close-packed array, in which case it would compete for volume with the rock and regolith samples. However, since the gas sample could be stored in a container of any shape, a more attractive possibility would be to store it in some of the noncylindrical volume of the OS. If the sample tube canister described above is cylindrical, and the OS is spherical, there would be extra volume around the sides, top, and bottom of the cylindrical canister. This volume is not (easily) usable for solid samples, but it may be ideal for one or more gas samples.

7.4.3. Organic blanks and calibration standards. A critical aspect of returned sample science, especially for biologyrelated investigations, would be recognizing false positives, including organics/microbes from Earth that make the round trip as well as organics/microbes from Earth that enter the analytical process after the samples are returned to Earth. Correct analytical laboratory procedure requires a system of carefully designed positive control standards and negative control standards, otherwise known as blanks. The use of blanks in organic/microbiological analysis of Apollo lunar samples was pioneered in the Lunar Receiving 
Laboratory under the protocols designed by the Baylor University College of Medicine (1967). We reinforce the recommendation of Rummel et al. (2002) that NASA "Invest significant time in the design of controls and blanks, as early in protocol development as possible."

The launching of flight instruments to Mars capable of organic analysis has required that blanks be used on the spacecraft to provide a baseline against which to judge any detection. The Phoenix spacecraft carried an Organic-Free Blank designed to be sampled and carried through the entire analytical chain of the Thermal and Evolved Gas Analyzer (Ming et al., 2008). MSL will carry five blocks of Organic Check Material to assess contamination levels through endto-end sample handling tests on Mars (Summons et al., 2011). For the MSR Campaign, analytical standards and blanks may need to be sent round-trip, commencing with a samplecaching mission. The design of the cache, as well as any in situ tests to detect organics or microbiology, would need community debate in order to optimize standards and blanks that may be introduced into the sample chain.

FINDING \#33: Some sample spaces in the canister (three seems like a reasonable initial planning figure, but this needs a carefully structured follow-up discussion) should be set aside for blanks/standards as a reserve against the outcome of future MSR scientific planning activities

7.4.4. Sample sealing and preservation. Adequate sealing of the sample tubes is necessary to preserve the samples while cached on the surface of Mars and during transport to Earth. For example, to ensure that sedimentological investigations are not compromised, delicate textures found in sedimentary layers should remain undisturbed (potentially for many years and during transport to Earth). For mineralogical and geochemical investigations, it would be necessary to preserve evidence for any volatile-bearing minerals and to avoid cross contamination as well as contamination from an outside source. For biological investigations, any biological matter must be preserved but not contaminated. Atmospheric science requires that the atmospheric sample does not leak and would not be contaminated by gases evolved from rock samples. Accordingly, it would be necessary to seal samples as they are collected. Some of the most important sample preservation considerations include

- Minimizing alteration of samples, for example, heating and crushing

- Considering how a sample tube would be opened in the Earth receiving facility

- Considering the dirty, dusty, and at times windy environment on Mars and its effect on sealing

- Minimizing contamination of the sample, for example, considering the materials being used for sealing

- Minimizing cross contamination between collected samples

- Monitoring temperature fluctuations

- Evaluating the impact acceleration on Earth

- Preserving volatiles to the greatest degree possible

Some of the possibilities for sample tube sealing are described in greater detail below. There is a trade-off between the type and degree of sample sealing and the potential for con- tamination. Thus, to achieve the science objectives for samples of unaltered igneous rocks, concerns about loss of volatiles (notably water-see below) is limited, but concerns of contamination from certain sealing agents (e.g., pure metals) could be very considerable. There are also significant concerns about organic contaminants that could affect our ability to achieve past and extant life objectives. At the other extreme, to achieve science objectives related to evaluating the martian surface for resources, of which water is arguably the most important, concerns about contamination are limited, whereas the need to seal against any water loss or gain would be absolutely critical. Concerns about volatile loss are also very high for any questions related to life and for those questions that include any processes involving fluid alteration.

Volatile species expected in the samples potentially include a wide variety of H-C-O-S-N species (e.g., phyllosilicates, sulfates, carbonates) that are entrapped in minerals, found along grain boundaries, and potentially found in inclusions. It is consistent with current planetary protection policy to sample ice, but only if the spacecraft is clean enough (which would impose additional mission cost). We do not believe that the scientific value of sampling ice for the MSR Campaign could justify this incremental expense. Much could be learned about the aqueous history of Mars and the potential for life by examining water, hydrogen, and deuterium. Water is a critical ingredient for life. Thus, movement of any water would provide especially significant science impact.

A second sealing issue arises at the time of assembling the sample cache with the atmospheric gas sample into the OS in preparation for transit to Earth. Thus, at the time of this assembly it would be highly desirable to seal the entire canister such that the canister would remain effectively at 1 martian atmosphere pressure and composition. The reason for this is that, during the extended transit time in space and residence in Earth's atmosphere, there would be considerable pressure and compositional gradients between the sample tubes and the ambient environment. Without sealing, such gradients would promote diffusion and transport of volatile materials into or out of the sample tubes.

FINDING \#34: The samples must maintain their scientific integrity while cached on the martian surface (potentially for many years) and during transport to Earth. A key is adequate sealing of the sample tubes.

FINDING \#35: The volatile species for which limiting mass transfer (in/out of the sample tubes) would be most valuable is water.

FINDING \#36: It would be scientifically desirable to seal the canister before leaving the martian surface (rather than in orbit), so as to avoid a significant pressure differential across the sample tube seals during transit.

7.4.5. Sealing concepts and materials. Mechanical sealing of the sample tubes is a fundamental feature of the design of the SHEC (Backes et al., 2010, 2011; Younse et al., 2010). Within the system, there is also the opportunity where the tube could be transferred to a sealing station and the mechanical plug is pushed farther into the tube to contact the sample, which would enable an estimate of the volume of the acquired sample and prevent the sample from moving in the tube during subsequent phases of the mission. At this point in the process, there 
Table 12. Sample Tube Sealing Concepts

CAP concepts for sample tube sealing

Press cap: press a cap onto the top of the tube leaving radial pressure. Might include indium surfaces and heating of indium.

Solder cap: solder a cap onto top of the tube.

Screw cap: screw a cap onto top of the tube to provide a pressure seal.

Indium knife: push an indium lined cap into an indium lined sharp edge on the tube; need to maintain pressure to help indium bond. Variations include means to clean away dust or oxidation layers on indium surfaces, e.g., press knife edge through membrane, scrape knife edge.

Shape memory alloy (SMA) cap
PLUG concepts for sample tube sealing

Cork: Press a plug into the end of the tube; the plug or tube deforms to cause pressure seal. Could line surfaces with indium (or warmed indium) to improve seal.

Solder Cork: Press a plug into the top of the tube. Then melt solder at top of plug to fill gaps between plug and tube via capillary action. The plug and/or tube might be pre-tinned (coated with solder material).

Tube clamp: Release inner spring to provide axial pressure seal; could include indium (or warmed indium) surfaces to improve seal.

Screw plug: screw a plug into the top of the tube to provide a pressure seal; could include indium surfaces to improve seal.

Shape memory alloy (SMA) plug: SMA plug that is heated to change metal phase and apply pressure seal would be an opportunity to consider additional means for isolating the sample from environments the tubes would experience over subsequent years while on the surface of Mars, in orbit about Mars, in transit to Earth, in the reentry and impact phases on Earth, and during the subsequent recovery period before the samples finally reach the SRF.

In general, the level of sealing could range from the mechanical seals designed to prevent movement of the sample within the tube and particle escape, to some level of hermetic sealing that would contain gases at a specified leak rate up to ultrahigh vacuum sealing (Table 12). The combination of sealing level and permissible materials, as well as the considerations listed above, present a significant range of technical and complexity challenges (i.e., risk and cost) to the development of adequate sealing levels and must be balanced against the preservation of specific measurement objectives of the MSR Campaign.

The history of compromised sample containers obtained in the Apollo mission (Allton, 1989) provides insights into the challenge of obtaining and preserving pristine samples. Based on this experience and subsequent research (e.g., Allen et al., 2011, and references therein), a variety of sample seals are possible:

- Teflon is a good choice for seal material; because of its high chemical inertness and thermal stability, the chance of chemical interaction/degradation/contamination is relatively low. Moreover, the potential (although unlikely) contaminants induced by Teflon would be fluorinated organics, which are easy to discriminate from martian organics. Nevertheless, the mere existence of organic molecules in the sealing materials could raise some concern. Teflon is one of the very few materials routinely used in the curation of extraterrestrial samples.

- Metals, especially copper, are traditionally used for gaskets (especially for gas samples). However, we need to assess the degree to which they may be oxidized and play a catalytic role once in contact with the martian samples.
- Indium is another known gasket material, whose sealing properties would need to be evaluated against the consequences of sample contamination in this area. Preliminary interaction with CAPTEM (Sept. 2011) may indicate that the value of the seal is more important than the value of protecting indium trace element geochemistry.

FINDING \#37: Materials used in the sealing process need to be compatible with the planned measurement objectives. Seals made of Teflon are an example of such a material.

\section{Conclusions}

\subsection{Summary}

(1) The MSR Campaign should address eight major, community-developed science objectives. The most important objective by far relates to determining whether evidence of past life or prebiotic chemistry exists in the examined materials.

(2) To answer the complex questions associated with the highest-priority scientific objectives of the MSR Campaign would require sample suites that are carefully selected through a process of comprehensive in situ science that also would provide critical context for sample analyses back on Earth.

(3) The total number of rocky samples that would be needed to address the objectives is $30-35$. The approximate mass per rock sample needed for analyses on Earth is $15-16 \mathrm{~g}$. Additionally, one or two gas samples would be required.

(4) There are multiple potential landing sites on Mars where it appears possible to meet the proposed science objectives of the MSR Campaign. To access these sites and sample the desired rocks, the mission may need to be able to tolerate some hazards in the landing ellipse (or have an ellipse small enough to avoid the hazards) and be able to traverse beyond the ellipse. 
(5) To achieve the in situ science and assemble the necessary sample suites, the proposed 2018 joint rover should have the field exploration capabilities defined by the E2E-iSAG.

\subsection{Recommendation for future work}

During the deliberations of the E2E-iSAG, it became apparent that there is a considerable amount of additional research and other effort required in preparation for the MSR Campaign. These can be conveniently divided into tasks best addressed by MEPAG and by NASA/ESA at both the programmatic level and in the area of research/development.

\subsubsection{MEPAG-related tasks.}

(1) Considering the prioritized objectives for the MSR Campaign, determine the sample contamination issues that would affect the scientific measurements to be made on Earth (parts of this should be worked jointly with planetary protection, CAPTEM, NASA Astrobiology Institute, other).

(a) Plan for quantitative contamination specifications that would need to be applied to all relevant parts of the MSR Campaign.

(b) Plan for positive and negative control standards, and where they need to be introduced into the sample chain, in order to document the state of contamination at specific times in the proposed rover's activity. This may involve a block of organicfree standard material that needs to be positioned within the robotic arm's workspace, and if so, the configuration engineers would need to know about this early in the design process.

(2) Evaluate and develop life-detection investigation and measurement strategies to be carried out on the returned samples (worked jointly with planetary protection, MEPAG, other).

\subsubsection{Programmatic issues (NASA and ESA)}

(3) As aggressively as possible, conduct a landing site qualification and prioritization process. This is crucially important while Mars Reconnaissance Orbiter is still in service!

(4) Determine the approximate depth of regolith sampling required as input to planning for an eventual human mission to the martian surface.

\subsubsection{Research work (NASA and ESA)}

(5) General research on ways to reduce needed sample mass, including

(a) Increased instrument sensitivity

(b) More efficient sample preparation, specifically including polished section manufacture

(c) Use of same sample material in sequential analyses.

(6) Improve understanding of the likely density range of returned materials, especially for martian sedimentary rocks.
(7) Using terrestrial analog sites, compare ancient hydrothermal/low- $T$ fluid alteration environments to ancient sedimentary environments for prospects of finding signs of ancient life.

(8) Conduct systematic research into issues relating to sample preservation. The issues related to heating/ cooling, volatile loss, and sample contamination were most obvious to the E2E team, but there likely are other issues.

\subsubsection{Engineering development (NASA and ESA)}

(9) Develop improvements in hazard avoidance capabilities and improved landing accuracy.

(10) Enhance rover operations efficiency (e.g., increased autonomy) and increase rover speed, to optimize productivity within a constrained lifetime.

(11) Develop and test drilling capabilities by using a library of relevant sample analogues to ensure adequate drill bit lifetime and sample quality.

(12) Optimize end-to-end sample handling to ensure mechanical core integrity and scientific quality until analysis.

(13) Research, development, and testing of sample sealing mechanisms, gaseous transmission rates across seals of different types, and evaluations of seal longevity.

(14) Additional study is needed to understand the implications to MSR science objectives of exposure of samples to spacecraft-induced magnetic fields and radiation.

(15) Development of a rock abrasion tool that has the capacity for a much larger number of uses than has been attempted on any prior mission.

\section{Appendices}

\section{A1. Glossary}

Aeolian deposits: any accumulation of wind-blown sediment that occurs in recognizable bedforms or morphologies.

Airfall dust: fine-grained material that has settled from the atmosphere.

Altered rocks: general category for materials that include any igneous, sedimentary, or metamorphic materials that have been secondarily altered by fluids passing through them.

Astrobiology: used as synonym of exobiology in this report.

Bioburden: a quantitative estimate of the number of viable microorganisms or viable biomass in or on a device, surface, or raw material. Buried bioburden indicates specifically the bioburden that is not accessible to surfacesterilizing influences.

Exobiology: used as synonym of astrobiology in this report.

Extrusive/effusive: rock that solidified after reaching the surface.

Felsic: rock consisting entirely of feldspars, feldspathoids, and/or quartz.

Float: isolated displaced fragments of rock on the surface.

Hydrothermal deposits-sediments: geological materials deposited at the surface from hot circulating fluids derived from magmatic or volcanic activity. 
Hydrothermally altered rocks: rocks altered by magmatic or volcanic activity; typically originating from higher- $T$ processes. In contrast, the term low-temperature fluid-altered rocks includes those related to subsurface alteration by meteoric fluids, that is, fluids derived ultimately from surface via recharge, for example, low- $T$ serpentinization, carbonate fracture mineral formation.

Igneous: rock formed by solidification from a molten or partially molten state.

Intrusive: rock that solidified before reaching the surface.

Regolith: the entire layer of fragmental and loose, incoherent, or unconsolidated rock material of any origin that mantles more coherent bedrock

(Sample) Suite: a set of samples connected by some sort of relationship.

Soil: any loose, unconsolidated material that can be distinguished from rocks, bedrock, or strongly cohesive sediments but has no singular origin.

Subaqueous sediments/Subaqueously deposited surface sediments: include those deposited by standing or flowing surface waters (such as a lake), or by groundwater (e.g., playas, tufa-style deposition).

Ultramafic: rock containing virtually no quartz or feldspar and consisting entirely of Fe-Mg silicates, metallic oxides, and sulfides.

Xenolithic: pertaining to rock fragments that are foreign to the igneous rock in which they occur.

\section{A2. Charter of This Study}

The proposed Mars sample return campaign would involve three flight elements: (1) sample collection/caching, (2) launch of the sample cache into Mars orbit, (3) collection and return to Earth of the sample cache. A fourth element would involve transport of the sample cache to a sample receiving facility (SRF). The proposed scientific objectives of this fourelement set of activities can be thought of in two categories:

(1) Science that would be derived from the overall campaign, culminating in the study of the returned samples on Earth, and

(2) Science that would be accomplished by each mission element at Mars, in support of the campaign goals, by means of instruments that might be present on the individual flight elements.

Planning for the second category needs to be considered one mission at a time and be linked to specific constraints related to planetary dynamics, mass, energy, and the financial environment of the time. This can be carried out through focused MEPAG Science Analysis Group (SAG) discussions and through mission-specific Science Definition Teams (SDT). However, each of the four elements would need to support overall MSR Campaign objectives that are broader than the individual missions themselves. Therefore, these overall objectives need to be understood in sufficient detail at an early stage in order to plan correctly for the roles of each of the component missions. This is the focus of the science analysis requested in this charter by MEPAG.

\section{A2.1. Charter assumptions}

(1) Assume that the "campaign-level" science objectives are derived from NRC reports (e.g., An Astrobiology
Strategy for the Exploration of Mars) and from the analyses by MEPAG Science Analysis Groups: ND-SAG (2008), MRR-SAG (2009), 2R-iSAG (2010).

(2) Assume that the MSR Campaign would consist of several flight elements (as described in presentations to MEPAG and the Planetary Decadal Survey), each of which must have a "controlled appetite" in areas such as mission instrumentation and sample preservation. Further assume that:

(a) The proposed 2018 rover mission would prepare one or more caches of carefully selected samples for return to Earth from a well-characterized site.

(b) The cache would be retrieved by a later "Fetch" rover, delivered to the surface on a platform with the Mars Ascent Vehicle that would return the samples to Mars orbit.

(3) Assume that the following sample acquisition functionality is available to the MSR Campaign (note that these are planning assumptions, not decisions):

(a) At least 20 encapsulated surface or subsurface samples of at least $\sim 10 \mathrm{~g}$ each, in addition to any necessary positive and negative control standards, to be scientifically selected and packaged using the instruments on the proposed MAX-C/ExoMars.

(b) One or more regolith samples collected from the immediate vicinity of the MSR lander by a deck- or body-mounted sampling system.

(c) One atmospheric gas sample collected into a valved, pressurized container. The combination of volume and pressure is TBD.

\section{A2.2. Methodology and approach}

\section{A2.2.1 Requested tasks.}

(1) MSR Campaign science objectives. Based on previous work (e.g., references in Assumption \#1 above), consolidate and prioritize a reference set of "campaignlevel" science objectives, from which the science-related requirements for the individual flight missions could be derived, and trades between them could be worked. Particular detail is required at this stage in areas that would affect the proposed 2018 sampling mission.

(2) Derived criteria. Map each reference MSR Campaign science objective to specific requirements (within the context of Assumption \#2 above) regarding (1) sample acquisition and handling and (2) site selection criteria. This activity should include (where possible) identification of "threshold" criteria and discussion of the scientific value gained by meeting additional requirements. Specific points to consider are

\section{(a) Samples:}

(i) Relative priorities for sampling different rock types (e.g., how diverse should/must the sample collection be?)

(ii) Value of subsurface material that may be provided by an ExoMars-type drill for inclusion in the sample cache (e.g., the value of cuttings as a sample or the kind of encapsulation needed) 
(iii) Nature and priority of regolith samples (e.g., grain size to be captured)

(iv) Nature and priority of gas samples (e.g., value of headspace gas)

(b) Instrumentation: Threshold capabilities required for adequate in situ characterization needed to support sample selection.

(c) Landing site criteria: Threshold landing site scientific attributes (those that must be present for any site to be considered), as well as qualifying scientific attributes that might make a candidate site more attractive from the point of view of each MSR Campaign science objective.

(i) Are there suitable candidate sites for the MSR Campaign in the $5 \mathrm{~S}$ to $25 \mathrm{~N}$ latitude band at elevations less than $-1 \mathrm{~km}$ ?

(ii) What is the value of going to sites outside this latitude band?

(3) Reference landing sites. To assist in planning the engineering of the landed elements of the MSR Campaign, identify several reference landing sites of interest that contain the proposed attributes. The purpose of these sites is to help the engineers design the mission elements in a way that at least some sites of interest could be accessed. Note that these reference sites will not carry any formal status; there will be an independent landing site competition.

(4) Inputs to technology planning. Assess the primary implications of the results of the above two tasks (\#2-3) for technology planning. For example, what are the implications for priorities in the areas of EDL capability, hazard avoidance, and mobility?

\section{A2.2.2. Methods.}

- The iSAG is asked to conduct its business primarily via telecons, e-mail, and/or web-based processes. At least one face-to-face meeting is encouraged, given the complexities of issues listed above.

- The Mars Program Office at JPL will provide logistical support.

\section{A2.2.3. Deliverables, schedule.}

- The SAG is expected to begin its discussions by August 2010.

- Lead a discussion on the E2E-iSAG's essential charter issues at the MEPAG meeting of September 30-October 1,2010 , giving the community a chance to provide input.

- A midterm report in PowerPoint format by December 1, 2010, which will be delivered and discussed at a town hall meeting at the Fall AGU conference.

- A final white paper report by the Spring 2011 MEPAG meeting (tentatively June, 2011), and a major presentation/discussion of that report.

\section{A3. Scientific Risk for the MSR Campaign}

While MSR has many science objectives, the main thrust would be to bring samples to Earth and examine them for evidence of past or present life and prebiotic chemistry. Return of samples from Mars is an endeavor with significant risks related to the uncertainties of a pioneering mission. As with all trailblazing efforts, the proposed MSR Campaign has the potential for extraordinary high rewards. Discovery and characterization of another form of life, whether extinct or extant, would be a transforming event in the history of science.

There are several different kinds of risks. There are political risks. The current plan of caching samples in the expectation that a future, as yet unfunded, mission would return the samples has obvious political risk. The distribution of samples and the type of scientific work permitted on political and ethical grounds may also bring uncertainty to the mission. Collecting and caching samples on Mars, rendezvousing with the cache, and returning it safely to Earth are complex and difficult endeavors, with significant engineering risks. Discussion of both political and engineering risks is outside the charter of this group. However, we are cognizant of several science risks. In Table A1 some risks are listed and steps that might be taken to alleviate them.

It may be that life never started on Mars or, if it did, no evidence of it survives in the landing area selected for the MSR Campaign. Fortunately, the value of returned samples is not restricted to the search for life. Almost every aspect of Mars' science, including climate change, atmospheric evolution, geological evolution, and prebiotic chemistry would be significantly advanced by the acquisition of samples. With samples in hand, the analytical capabilities of laboratories worldwide could be devoted to better understanding of these issues. For example, an outstanding question-that may be viewed as a scientific risk-is whether life on other planets could be defined in the same manner as life on Earth or if it must be defined by other unusual features beyond our scientific experience. Multiple analyses of martian samples on Earth would allow us to use the scientific method to address this question.

Another science risk is that our pre-landing model of the geology of the sample site may be incorrect so that the sample site does not yield the type of samples that we expect. This risk could be offset by continuing an active remote sensing program and supporting a landing site selection process with broad participation of the science community. Another science risk is that the optimum science sites do not meet the engineering criteria for safe landing. This risk could be offset by a combination of the choice of landing site and a "go-to" capability so that the landing could be in a safe area and the rover could travel to the science-rich regions.

Another risk is that the site may be of the appropriate type but we are unable to recognize the most valuable sample(s) at the site. The sampling vehicle must, therefore, have a diverse array of analytical instruments as well as a tool(s) to expose a surface of the sample that is unaffected by surface weathering. In this way, crucial indicators of rock type (mineralogy, geochemistry, and texture) and potential habitability (e.g., organic content and texture) would be recognized as the landing site is explored. Furthermore, the vehicle must operate for long enough that sufficient information could be collected to select the best samples for caching.

A risk associated with sampling and return of samples from Mars relates to the unknown chemical and mechanical integrity of the samples. Again, including analytical 
instruments and tool(s) on the rover to remove weathering rinds would help alleviate the risk related to sampling unknown materials or materials with different chemical and mechanical properties. In addition, a comprehensive testing program would help us understand the nature of the changes that are undergone during sample acquisition, storage, and transport to Earth.

Engineering analyses are not part of this report, but a detailed investigation of the future "trades" necessary to contain complexity, cost, and overall feasibility is warranted because engineering may pose risk to the science outcomes. For example, the risks associated with caching would be reduced if two separate caches were left on the surface for later retrieval (NRC, 2011). Two caches would provide redundancy if a cache is lost due to a later mission failure and would not necessitate reflight of the caching part of the mission. However, it is uncertain if the extra investment required for two caches is balanced by the reduction in risk. In addition, there is considerable uncertainty regarding coring and cross-contamination when using the same bits and the means to correct errors during core break-off.

It is possible that the most interesting sites may be encountered late in the landed mission after most of the sample slots have been filled. There would be pressure to fill the sample slots early in the mission as in situ characterization of the accessible materials yields new insights and for fear that the rover lifetime may be limited. This risk could be offset by having a significant reserve of sample slots and the ability to replace collected samples with newly acquired samples.

It is critical that the public is educated on the potential risks of samples returned from Mars to health, and the planetary protection (quarantine) measures to be used. Sample return and analysis would require a good under- standing of these issues, and it is essential that facilities would be in place with trained staff well in advance of sample return. Planning should begin for a SRF location as soon as possible. In addition, it is necessary that the analytical capabilities in the SRF would be adequate for evaluating planetary protection issues as well as evaluating major questions about Mars.

\section{A4. Planetary Protection Issues/Opportunities}

\section{Introduction}

Planetary protection encompasses both protecting Mars from terrestrial contamination (forward protection) and protecting the Earth from potentially harmful materials returned from Mars (backward contamination). A major concern of forward contamination is to limit introduction of any terrestrial materials onto the martian surface that might compromise detections of any extinct or extant life-forms or traces of any prebiotic chemistry by future missions. This is accomplished mainly through internationally agreed-upon controls on sterilization and bioburden that depend among other things on the mission type and target body (COSPAR, 2011). The main concern of back protection is to contain safely and usefully any returned samples until they are demonstrated to be harmless.

\section{Information}

When the returned samples are evaluated in Earth laboratories, the needs of both planetary protection and scientific research can only be met by making measurements on the samples. Since the quantity of sample material available would be highly limited, it would be crucial to develop an understanding of the measurements that would be in common to planetary protection and science. Although planetary

Table A1. Examples of Scientific Risks of the MSR CAmpaign

Ref. Science Risk Possible mitigation strategy

1 Safe landing area may be inadequate to achieve returned sample science objectives.

Site may not be correctly interpreted from orbit, and for this reason not have the samples we want/need to meet MSR science goals

3 The landing site has the samples we want/need, but we are not able to recognize them.

4 Sample retrieval may not happen because of engineering, funding, or political issues

5 Most interesting samples may be encountered late in the mission and are not cacheable.

6 Public perception of danger of martian samples may hinder approval of their return and analysis

$7 \quad$ Public perception of failure if no life found

8 Chemical and mechanical integrity of samples compromised during acquisition, storage, and transport

9 Samples compromised by sample handling procedures after receipt on Earth
Have a GOTO capability. Improve landing accuracy. Add EDL capability to land at more hazardous sites. Keep altitude/latitude options as wide as possible.

Keep up an active orbital imaging program, and carry out a landing site selection process that is as open as possible. Choose site that has diverse science goals, not narrowly focused.

Ensure that the instruments necessary to identify the samples of interest are present and that the rover has enough time to use them.

Choose sites and instruments that provide good science independent of sample return

Have adequate reserves of sample tubes and capability of replacement in cache

Educate public on risks and planetary protection measures

Emphasize multiple science goals (life, climate change, planetary evolution, abiotic chemistry)

Comprehensive testing program with different geological materials, different acquisition techniques, etc.

Construct SRF and supporting facilites, staff and train paticipating personnel, well before Earth return 
protection and science may have different needs for accuracy and precision in returned sample measurements, it should be possible to determine which one has more stringent requirements for certain classes of information and to produce at least some of the data in a coordinated way. This would be an important strategy to conserve sample mass. Examples of data of relevance in both arenas might include mineralogy, texture, and organic carbon concentration and speciation. There would also be data required by both planetary protection and science that are not in common.

The justification for the mission is to conduct exciting and cutting-edge science. If this cannot be achieved, the mission should not be flown. The goals of planetary protection are also critical, and if they cannot be met, the mission also should not be flown. Because of engineering considerations, the amount of sample returned to Earth would be quite limited. Thus, there is very high pressure for both science and planetary protection to learn how to achieve their purposes with as small a quantity of sample as possible. Finding ways to minimize destructive sample testing and to maximize sample reuse (so that sample material from an initial use could all, or in part, also be used for a subsequent use) would be essential. Exactly how the sample-related demands between science and planetary protection would be balanced is deferred to future panels, but we encourage those panels to work cooperatively together.

\section{Issues of sample size and diversity}

As discussed at length in this report, to have the best chance of finding the signs of past or present life, sample variety must be maximized. Multiple small samples (perhaps in the $2-5 \mathrm{~g}$ range) would almost certainly be more useful than a few large (50 g) ones. However, the smaller the samples, the more difficult it may be to render judgments on the safety of individual samples and the collection as a whole. This trade-off between the desirability of heterogeneity from the point of view of science and the possible difficulty of dealing with heterogeneity by planetary protection must be studied and a proper balance found.

It is possible that traces of life on Mars may be very rare and heterogeneously distributed, and the concentration of organics in the soil may be very low. Thus, it is essential to avoid biological contamination as well as organic contamination of Mars and of the collected samples. Some sciencedriven limits for organic and nonorganic contaminants are given by Mahaffy et al. (2004) and MacPherson et al. (2005). The tables in these reports need to be updated to reflect more modern understanding of the needs and opportunities in the area. The proposed missions of the MSR Campaign would ultimately be flown with a single set of contamination control requirements, so it is mandatory that science and planetary protection converge on acceptable values.

The most recent and comprehensive summary of how returned martian samples might be treated on their return to Earth from a planetary protection perspective is that by Rummel et al. (2002). Analytical techniques have, however, evolved significantly since this study, and an update of this document is appropriate. Although the return of martian samples to Earth may be decades away, it is vital that sample requirements be understood in the early design stages of the sample acquisition system. We need to know what requirements planetary protection protocols would impose for such things as sample mass, bioburden limits, nonbiological contamination limits, whether and what type of bioassays are needed, and so forth. We therefore recommend that, as the science requirements for sample return are being formulated, a planetary protection panel be established to update or replace the Rummel et al. (2002) Draft Test Protocol in order that the sample acquisition system could be designed to satisfy both the science and the planetary protection requirements.

\section{A5. Reference Landing Sites}

On the MEPAG Web site: http://mepag.jpl.nasa.gov/reports/ Appendix_5_Landing_sites_v6.pdf

\section{Acknowledgments}

The team acknowledges significant input from Mike Hecht (in the area of regolith science), Bob Pepin, Ingo Leya, and John Jones (in the area of gas chemistry), Ben Weiss (in the area of paleomagnetism), Margaret Race, John Rummel, Karen Buxbaum, and Cassie Conley (in the area of planetary protection), Paul Backes and team (in the area of sample acquisition/caching), Sidney Hemming, Elizabeth Pierce, and Troy Rasbury (in the area of sedimentary component analysis and sample sizing), and Bruce Jakosky and Paul Mahaffy (in the area of gas chemistry results expected from MAVEN and MSL). We acknowledge review comments or significant discussions from/with (in alphabetical order): Deborah Bass, John Bridges, Charles Budney, Phil Christensen, Max Coleman, David Fernández-Remolar, Danny Glavin, Fred Goesmann, John Grotzinger, Emmanuelle Javaux, Jeff Johnson, Lisa May, Hap McSween, Dick Morris, Jack Mustard, Paul Niles, Jim Papike, Caroline Smith, Andrew Steele, Michael Velbel, Frances Westall, Charles Whetsel, James Wray, and Rich Zurek. Early drafts of this report were discussed at town hall-style sessions at the December 2010 AGU fall meeting and the MEPAG meeting in Lisbon (June, 2011), and also with the Planetary Protection Subcommittee (Jan. 2011) — many useful comments were received at these venues. Part of this work was carried out at the Jet Propulsion Laboratory, California Institute of Technology, under a contract with the National Aeronautics and Space Administration. Travel support from NASA, ESA, and the Canadian Space Agency for various team members is gratefully acknowledged. C.D.K.H. acknowledges the University of Alberta for sabbatical leave and a Smithsonian Institution Senior Research Fellowship.

\section{Abbreviations}

2R-iSAG: 2-Rover International Science Analysis Group, a 2010 MEPAG study team.

AGU: American Geophysical Union.

ALD: Analytical Laboratory Drawer, a group of instruments within the ESA Pasteur payload.

CAPTEM: Curation and Analysis Planning Team for Extraterrestrial Materials, a committee within the NASA advisory system.

CAT: computer-aided tomographic.

CLUPI: Close-up Imager, an instrument within the ESA Pasteur payload. 

tion.

COMPLEX: Committee on Planetary and Lunar Explora-

CRISM: Compact Reconnaissance Imaging Spectrometer for Mars, an instrument on the 2005 Mars Reconnaissance Orbiter mission.

E2E-iSAG: End-to-End International Science Analysis Group.

EDL: entry, descent, and landing.

EEV: Earth entry vehicle.

EMTGO: ExoMars Trace Gas Orbiter, a proposed mission to Mars baselined for launch in 2016.

GCR: galactic cosmic rays.

Go-to: referred to landing sites; the site of interest is outside the landing ellipse.

iMARS: International Mars Architecture for the Return of Samples, a 2008 committee of the International Mars Exploration Working Group.

LD-BH: life-detection and biohazard.

LMC: Life Marker Chip, an instrument within the ESA Pasteur payload.

Mars-XRD: Mars X-Ray Diffractometer, an instrument within the ESA Pasteur payload.

MATMOS: Mars Atmospheric Trace Molecule Occultation Spectrometer, an instrument on the proposed 2016 EMTGO mission.

MAV: Mars Ascent Vehicle.

MAVEN: Mars Atmosphere and Volatile Evolution Mission, a mission to Mars scheduled for launch in 2013.

MAX-C: Mars Astrobiology Explorer-Cacher, a mission concept proposed by MRR-SAG (2009).

MEPAG: Mars Exploration Program Analysis Group.

MER: Mars Exploration Rover, a 2003 mission to Mars.

MGS: Mars Global Surveyor, a 1996 mission to Mars.

MOMA: Mars Organic Molecule Analyser, an instrument within the ESA Pasteur payload.

MRR-SAG: Mid-Range Rover Science Analysis Group, a 2009 MEPAG study team.

MRSH: Mars Returned Sample Handling.

MSL: Mars Science Laboratory, a 2011 mission to Mars.

MSR: Mars sample return.

MSR-L: MSR Lander, a mission element of the proposed

MSR Campaign concept.

MSR-O: MSR Orbiter, a mission element of the proposed MSR Campaign concept.

ND-SAG: Next Decade Mars Sample Return Science

Analysis Group, a 2008 MEPAG study team.

NRC: National Research Council.

OS: orbiting sample container.

PanCam: panoramic camera system, an instrument within the ESA Pasteur payload.

SAM: Sample Analysis at Mars, an instrument on the 2011 MSL mission.

SHEC: Sample Handling, Encapsulation, and Containerization.

SNC: Shergotty, Nakhla, and Chassigny (a meteorite grouping).

SRF: sample receiving facility.

TES: Thermal Emission Spectrometer, an instrument on 1996 MGS mission.

WISDOM: Water Ice and Subsurface Deposit Information On Mars, an instrument within the ESA Pasteur payload.

\section{References}

Acuña, M.H., Connerney, J.E.P., Wasilewski, P., Lin, R.P., Mitchell, D., Anderson, K.A., Carlson, C.W., McFadden, J., Rème, H., Mazelle, C., Vignes, D., Bauer, S.J., Cloutier, P., and Ness, N.F. (2001) Magnetic field of Mars: summary of results from the aerobraking and mapping orbits. LGeophys Res 106:23403-23417.

Allen, C., Allton, J., Lofgren, G., Righter, K., and Zolensky, M. (2011) Curating NASA's extraterrestrial samples-past, present, and future. Chem Erde 71:1-20.

Allwood, A.C., Walter, M.R., Kamber, B.S., Marshall, C.P., and Burch, I.W. (2006) Stromatolite reef from the Early Archaean era of Australia. Nature 441:714-718.

Allwood, A.C., Grotzinger, J.P., Knoll, A.H., Burch, I.W., Anderson, M.S., Coleman, M.L., and Kanik, I. (2009) Controls on development and diversity of Early Archean stromatolites. Proc Natl Acad Sci USA 106:9548-9555.

Allton, J.H. (1989) Catalog of Apollo Lunar Surface Geological Sampling Tools and Containers, JSC-23454, LESC-26676, National Aeronautics and Space Administration, Washington DC.

Arvidson, R.V., Squyres, S.W., Anderson, R.C., Bell, J.F., III, Blaney, D., Brückner, J., Cabrol, N.A., Calvin, W.M., Carr, M.H., Christensen, P.R., Clark, B.C., Crumpler, L., Des Marais, D.J., de Souza, P.A., Jr., d'Uston, C., Economou, T., Farmer, J., Farrand, W.H., Folkner, W., Golombek, M., Gorevan, S., Grant, J.A., Greeley, R., Grotzinger, J., Guinness, E., Hahn, B.C., Haskin, L., Herkenhoff, K.E., Hurowitz, J.A., Hviid, S., Johnson, J.R., Klingelhöfer, G., Knoll, A.H., Landis, G., Leff, C., Lemmon, M., Li, R., Madsen, M.B., Malin, M.C., McLennan, S.M., McSween, H.Y., Ming, D.W., Moersch, J., Morris, R.V., Parker, T., Rice, J.W., Richter, L., Rieder, R., Rodionov, D.S., Schröder, C., Sims, M., Smith, M., Smith, P., Soderblom, L.A., Sullivan, R., Thompson, S.D., Tosca, N.J., Wang, A., Wänke, H., Ward, J., Wdowiak, T., Wolff, M., and Yen, A. (2006) Overview of the Spirit Mars Exploration Rover mission to Gusev Crater: landing site to Backstay Rock in the Columbia Hills. J Geophys Res 111, doi:10.1029/2005JE002499.

Backes, P., Lindemann, R., Collins, C., and Younse, P. (2010) An integrated coring and caching concept. In 2010 IEEE Aerospace Conference, Institute of Electrical and Electronics Engineers (IEEE), Piscataway, NJ, doi:10.1109/AERO.2010.5446985.

Backes, P., Younse, P., DiCicco, M., Hudson, N., Collins, C., Allwood, A., Paolini, R., Male, C., Ma, J., Steele, A., and Conrad, P. (2011) Experimental results of rover-based coring and caching. In 2011 IEEE Aerospace Conference, Institute of Electrical and Electronics Engineers (IEEE), Piscataway, NJ, doi:10.1109/AERO.2011.5747263.

Baker, V.R. (2001) Water and the martian landscape. Nature 412:228-236.

Bandfield, J.L., Glotch, T.D., and Christensen, P.R. (2003) Spectroscopic identification of carbonate minerals in the martian dust. Science 301:1084-1087.

Bandfield, J.L., Hamilton, V.E., Christensen, P.R., and McSween, H.Y., Jr. (2004) Identification of quartzofeldspathic materials on Mars. J Geophys Res 109, doi:10.1029/2004JE002290.

Banin, A. (2005) The enigma of the martian soil. Science 309:888890.

Barnhart, C.J. and Nimmo, F. (2011) Role of impact excavation in distributing clays over Noachian surfaces. J Geophys Res 116, doi:10.1029/2010JE003629.

Baylor University College of Medicine. (1967) Comprehensive Biological Protocol for the Lunar Sample Receiving Laboratory, Manned Spacecraft Center, National Aeronautics and Space Administration, Houston. 
Beaty, D.W., Allen, C.C., Bass, D.S., Buxbaum, K.L., Campbell, J.K., Lindstrom, D.J., Miller, S.L., and Papanastassiou, D.A. (2009) Planning considerations for a Mars sample receiving facility: summary and interpretation of three design studies. Astrobiology 9:745-758.

Becker, L., Antione, M., Cornish, T., Pinnick, V., and Cotter, R. (2010) The search for life on Mars using the Mars Organic Molecule Analyzer "MOMA" [abstract 2345]. In $41^{\text {st }}$ Lunar and Planetary Science Conference, Lunar and Planetary Institute, Houston.

Bell, J.F., III, Squyres, S.W., Arvidson, R.E., Arneson, H.M., Bass, D., Blaney, D., Cabrol, N., Calvin, W., Farmer, J., Farrand, W.H., Goetz, W., Golombek, M., Grant, J.A., Greeley, R., Guinness, E., Hayes, A.G., Hubbard, M.Y., Herkenhoff, K.E., Johnson, M.J., Johnson, J.R., Joseph, J., Kinch, K.M., Lemmon, M.T., Li, R., Madsen, M.B., Maki, J.N., Malin, M., McCartney, E., McLennan, S., McSween, H.Y., Jr., Ming, D.W., Moersch, J.E., Morris, R.V., Dobrea, E.Z., Parker, T.J., Proton, J., Rice, J.W., Jr., Seelos, F., Soderblom, J., Soderblom, L.A., SohlDickstein, J.N., Sullivan, R.J., Wolff, M.J., and Wang, A. (2004a) Pancam multispectral imaging results from the Spirit rover at Gusev Crater. Science 305:800-806.

Bell, J.F., III, Squyres, S.W., Arvidson, R.E., Arneson, H.M., Bass, D., Calvin, W., Farrand, W.H., Goetz, W., Golombek, M., Greeley, R., Grotzinger, J., Guinness, E., Hayes, A.G., Hubbard, M.Y., Herkenhoff, K.E., Johnson, M.J., Johnson, J.R., Joseph, J., Kinch, K.M., Lemmon, M.T., Li, R., Madsen, M.B., Maki, J.N., Malin, M., McCartney, E., McLennan, S., McSween, H.Y., Jr., Ming, D.W., Morris, R.V., Dobrea, E.Z., Parker, T.J., Proton, J., Rice, J.W., Jr., Seelos, F., Soderblom, J.M., Soderblom, L.A., Sohl-Dickstein, J.N., Sullivan, R.J., Weitz, C.M., and Wolff, M.J. (2004b) Pancam multispectral imaging results from the Opportunity rover at Meridiani Planum. Science 306:1703-1709.

Bertka, C.M. and Fei, Y.W. (1997) Mineralogy of the martian interior up to core-mantle boundary pressures. $[$ Geophys Res 102:5251-5264.

Bertka, C.M. and Holloway, J.R. (1994) Anhydrous partial melting of an iron-rich mantle. 2. Primary melt compositions at 15 Kbar. Contrib Mineral Petrol 115:323-338.

Bibring, J.-P., Langevin, Y., Mustard, J.F., Poulet, F., Arvidson, R., Gendrin, A., Gondet, B., Mangold, N., Pinet, P., Forget, F., and the OMEGA team. (2006) Global mineralogical and aqueous Mars history derived from OMEGA/Mars Express data. Science 312:400-404.

Bishop, J.L., Noe Dobrea, E.Z., McKeown, N.K., Parente, M., Ehlmann, B.L., Michalski, J.R., Milliken, R.E., Poulet, F., Swayze, G.A., Mustard, J.F., Murchie, S.L., and Bibring, J.-P. (2008) Phyllosilicate diversity and past aqueous activity revealed at Mawrth Vallis, Mars. Science 321:830-833.

Blinova, A. and Herd, C.D.K. (2009) Experimental study of polybaric REE partitioning between olivine, pyroxene and melt of the Yamato 980459 composition: insights into the petrogenesis of depleted shergottites. Geochim Cosmochim Acta 73:3471-3492.

Bogard, D.D. and Garrison, D.H. (1998) Relative abundances of argon, krypton, and xenon in the martian atmosphere as measured in martian meteorites. Geochim Cosmochim Acta 62:1829-1835.

Bogard, D.D. and Johnson, P. (1983) Martian gases in an Antarctic meteorite. Science 221:651-654.

Borg, L.E., Nyquist, L.E., Taylor, L.A., Wiesmann, H., and Shih, C.Y. (1997) Constraints on martian differentiation processes from $\mathrm{Rb}-\mathrm{Sr}$ and $\mathrm{Sm}-\mathrm{Nd}$ isotopic analyses of the basaltic shergottite QUE 94201. Geochim Cosmochim Acta 61:4915-4931.
Borg, L.E., Nyquist, L.E., Wiesmann, H., Shih, C.-Y., and Reese, Y. (2003) The age of Dar al Gani 476 and the differentiation history of the martian meteorites inferred from their radiogenic isotopic systematics. Geochim Cosmochim Acta 67:3519-3536.

Borg, L.E., Connelly, J.N., Boyet, M., and Carlson, R.W. (2011) Chronological evidence that the Moon is either young or did not have a global magma ocean. Nature 477:70-72.

Boston, P.J., Ivanov, M.V., and McKay, C.P. (1992) On the possibility of chemosynthetic ecosystems in subsurface habitats on Mars. Icarus 95:300-308.

Bouvier, A., Blichert-Toft, J., Vervoort, J.D., Gillet, P., and Albarede, F. (2008) The case for old basaltic shergottites. Earth Planet Sci Lett 266:105-124.

Boynton, W.V., Feldman, W.C., Squyres, S.W., Prettyman, T.H., Bruckner, J., Evans, L.G., Reedy, R.C., Starr, R., Arnold, J.R., Drake, D.M., Englert, P.A., Metzger, A.E., Mitrofanov, I., Trombka, J.I., D’Uston, C., Wanke, H., Gasnault, O., Hamara, D.K., Janes, D.M., Marcialis, R.L., Maurice, S., Mikheeva, I., Taylor, G.J., Tokar, R., and Shinohara, C. (2002) Distribution of hydrogen in the near surface of Mars: evidence for subsurface ice deposits. Science 297:81-85.

Brasier, M.D., Green, O.R., Jephcoat, A.P., Kleppe, A.K., Van Kranendonk, M.J., Lindsay, J.F., Steele, A., and Grassineau, N.V. (2002) Questioning the evidence for the Earth's oldest fossils. Nature 416:76-81.

Britt, D.T. and Consolmagno, G.J.S.J. (2003) Stony meteorite porosities and densities: a review of the data through 2001. Meteorit Planet Sci 38:1161-1180.

Burns, R.G. (1993) Rates and mechanisms of chemical weathering of ferromagnesian silicate minerals on Mars. Geochim Cosmochim Acta 57:4555-4574.

Cabrol, N. and Grin, E.A. (2010) Lakes on Mars, Elsevier, Amsterdam.

Cady, S.L. and Farmer, J.D. (1996) Fossilization processes in siliceous thermal springs: trends in preservation along thermal gradients. In Evolution of Hydrothermal Ecosystems on Earth (and Mars?), Ciba Foundation Symposium 202, edited by G.R. Bock and J.A. Goode, John Wiley and Sons, Chichester, UK, pp 150173.

Carr, M.H. (1996) Water on Mars, Oxford University Press, Oxford.

Carr, M.H. and Head, J.W., III. (2010) Geologic history of Mars. Earth Planet Sci Lett 294:185-203.

Chapelle, F.H., O'Neill, K., Bradley, P.M., Methé, B.A., Ciufo, S.A., Knobel, L.L., and Lovley, D.R. (2002) A hydrogen-based subsurface microbial community dominated by methanogens. Nature 415:312-315.

Chivian, D., Brodie, E.L., Alm, E.J., Culley, D.E., Dehal, P.S., DeSantis, T.Z., Gihring, T.M., Lapidus, A., Lin, L.H., Lowry, S.R., Moser, D.P., Richardson, P.M., Southam, G., Wanger, G., Pratt, L.M., Andersen, G.L., Hazen, T.C., Brockman, F.J., Arkin, A.P., and Onstott, T.C. (2008) Environmental genomics reveals a single-species ecosystem deep within Earth. Science 322:275-278. Christensen, P.R., Ruff, S.W., Fergason, R.L., Knudson, A.T., Anwar, S., Arvidson, R.E., Bandfield, J.L., Blaney, D.L., Budney, C., Calvin, W.M., Glotch, T.D., Golombek, M.P., Gorelick, N., Graff, T.G., Hamilton, V.E., Hayes, A., Johnson, J.R., McSween, H.Y., Jr., Mehall, G.L., Mehall, L.K., Moersch, J.E., Morris, R.V., Rogers, A.D., Smith, M.D., Squyres, S.W., Wolff, M.J., and Wyatt, M.B. (2004a) Initial results from the Mini-TES experiment in Gusev Crater from the Spirit rover. Science 305:837-842.

Christensen, P.R., Wyatt, M.B., Glotch, T.D., Rogers, A.D., Anwar, S., Arvidson, R.E., Bandfield, J.L., Blaney, D.L., Budney, 
C., Calvin, W.M., Fallacaro, A., Fergason, R.L., Gorelick, N., Graff, T.G., Hamilton, V.E., Hayes, A.G., Johnson, J.R., Knudson, A.T., McSween, H.Y., Jr., Mehall, G.L., Mehall, L.K., Moersch, J.E., Morris, R.V., Smith, M.D., Squyres, S.W., Ruff, S.W., and Wolff, M.J. (2004b) Mineralogy at Meridiani Planum from the Mini-TES experiment on the Opportunity rover. Science 306:1733-1739.

Christensen, P.R., McSween, H.Y., Jr., Bandfield, J.L., Ruff, S.W., Rogers, A.D., Hamilton, V.E., Gorelick, N., Wyatt, M.B., Jakosky, B.M., Kieffer, H.H., Malin, M.C., and Moersch, J.E. (2005) Evidence for magmatic evolution and diversity on Mars from infrared observations. Nature 436:504-509.

Christensen, P.R., Osterloo, M., Hamilton, V., Edwards, C., Wray, J., and Anderson, F.S. (2008) Aqueous mineral deposits in an ancient, channeled, equatorial terrain. In Mars Science Laboratory $2^{\text {nd }}$ Workshop, Pasadena, CA.

Clark, B.C., III, Baird, A.K., Rose, H.J., Jr., Toulmin, P., III, Christian, R.P., Kelliher, W.C., Castro, A.J., Rowe, C.D., Keil, K., and Huss, G.R. (1977) The Viking X-ray fluorescence experiment: analytical methods and early results. L Geophys Res 82:4577-4594.

Cockell, C.S., Lee, P., Osinski, G., Horneck, G., and Broady, P. (2002) Impact-induced microbial endolithic habitats. Meteorit Planet Sci 37:1287-1298.

Collins, C., Younse, P., and Backes, P. (2009) Planetary sample caching system design options [AIAA-2009-6506]. In AIAA SPACE 2009 Conference \& Exposition, American Institute of Aeronautics and Astronautics, Reston, VA.

COMPLEX. (2002) The Quarantine and Certification of Martian Samples, The National Academies Press, Washington DC.

COSPAR (2011) COSPAR Planetary Protection Policy (updated March 2011). Committee on Space Research (COSPAR), Paris. Available online at http://cosparhq.cnes.fr/Scistr/PPPolicy\% 20(24Mar2011).pdf.

Coulson, I.M., Beech, M., and Nie, W. (2007) Physical properties of martian meteorites: porosity and density measurements. Meteorit Planet Sci 42:2043-2054.

Dartnell, L.R., Desorgher, L., Ward, J.M., and Coates, A.J. (2007) Modelling the surface and subsurface martian radiation environment: implications for astrobiology. Geophys Res Lett 34, doi:10.1029/2006GL027494.

Dehouck, E., Mangold, N., Le Mouélic, S., Ansan, V., and Poulet, F. (2010) Ismenius Cavus, Mars: a deep paleolake with phyllosilicate deposits. Planet Space Sci 58:941-946.

Des Marais, D.J. (1996) Stable light isotope biogeochemistry of hydrothermal systems. In Evolution of Hydrothermal Ecosystems on Earth (and Mars?), Ciba Foundation Symposium 202, edited by G.R. Bock and J.A. Goode, John Wiley and Sons, Chichester, UK, pp 83-98.

DeVincenzi, D.L. and Bagby, J.R. (1981) Orbiting Quarantine Facility: the Antaeus Report, NASA SP-454, National Aeronautics and Space Administration, Washington DC.

Drake, B.G., editor. (2009) Human Exploration of Mars Design Reference Architecture 5.0, NASA-SP-2009-566, National Aeronautics and Space Administration, Washington DC.

Ehlmann, B.L., Mustard, J.F., Fassett, C.I., Schon, S.C., Head, J.W., III, Des Marais, D.J., Grant, J.A., and Murchie, S.L. (2008a) Clay minerals in delta deposits and organic preservation potential on Mars. Nat Geosci 1:355-358.

Ehlmann, B.L., Mustard, J.F., Murchie, S.L., Poulet, F., Bishop, J.L., Brown, A.J., Calvin, W.M., Clark, R.N., Des Marais, D.J., Milliken, R.E., Roach, L.H., Roush, T.L., Swayze, G.A., and Wray, J.J. (2008b) Orbital identification of carbonate-bearing rocks on Mars. Science 322:1828-1832.
Ehlmann, B.L., Mustard, J.F., Swayze, G.A., Clark, R.N., Bishop, J.L., Poulet, F., Des Marais, D.J., Roach, L.H., Milliken, R.E., Wray, J.J., Barnouin-Jha, O., and Murchie, S.L. (2009) Identification of hydrated silicate minerals on Mars using MROCRISM: Geologic context near Nili Fossae and implications for aqueous alteration. J Geophys Res 114, doi:10.1029/ 2009JE003339.

Elkins-Tanton, L.T., Hess, P.C., and Parmentier, E.M. (2005) Possible formation of ancient crust on Mars through magma ocean processes. J Geophys Res 110, doi:10.1029/2005JE002480.

Fairén, A.G., Davila, A.F., Lim, D., Bramall, N., Bonaccorsi, R., Zavaleta, J., Uceda, E.R., Stoker, C., Wierzchos, J., Dohm, J.M., Amils, R., Andersen, D., and McKay, C.P. (2010) Astrobiology through the ages of Mars: the study of terrestrial analogues to understand the habitability of Mars. Astrobiology 10:821-843.

Farmer, J. (1998) Thermophiles, early biosphere evolution, and the origin of life on Earth: implications for the exobiological exploration of Mars. I Geophys Res 103:28457-28461.

Farmer, J.D. (2000) Hydrothermal systems: doorways to early biosphere evolution. GSA Today 10:1-9.

Farmer, J. and Des Marais, D. (1999) Exploring for a record of ancient martian life. L Geophys Res 104:26977-26995.

Fassett, C.I. and Head, J.W., III. (2005) Fluvial sedimentary deposits on Mars: ancient deltas in a crater lake in the Nili Fossae region. Geophys Res Lett 32, doi:10.1029/2005GL023456.

Filiberto, J. and Treiman, A.H. (2009) Martian magmas contained abundant chlorine, but little water. Geology 37:10871090.

Fritz, J., Artemieva, N., and Greshake, A. (2005) Ejection of martian meteorites. Meteorit Planet Sci 40:1393-1411.

Garrison, D.H. and Bogard, D.D. (1998) Isotopic composition of trapped and cosmogenic noble gases in several martian meteorites. Meteorit Planet Sci 33:721-736.

Gary, M., McAfee, R., and Wolf, C.L., editors. (1972) Glossary of Geology, American Geological Institute, Washington DC.

Gellert, R., Rieder, R., Anderson, R.C., Brückner, J., Clark, B.C., Dreibus, G., Economou, T., Klingelhöfer, G., Lugmair, G.W., Ming, D.W., Squyres, S.W., d'Uston, C., Wänke, H., Yen, A., and Zipfel, J. (2004) Chemistry of rocks and soils in Gusev Crater from the Alpha Particle X-ray Spectrometer. Science 305:829-832.

Gilmour, J., Lyon, I.C., Johnston, W.A., and Turner, G. (1994) RELAX: an ultrasensitive, resonance ionization mass spectrometer for xenon. Rev Sci Instrum 65:617-625.

Golden, D.C., Ming, D.W., Schwandt, C.S., Lauer, H.V., Jr., Socki, R.A., Morris, R.V., Lofgren, G.E., and McKay, G.A. (2001) A simple inorganic process for formation of carbonates, magnetite, and sulfides in martian meteorite ALH84001. Am Mineral 86:370-375.

Golombek, M., Grant, J., Vasavada, A.R., Grotzinger, J., Watkins, M., Kipp, D., Noe Dobrea, E., Griffes, J., and Parker, T. (2011) Final four landing sites for the Mars Science Laboratory [abstract 1520]. In $42^{\text {nd }}$ Lunar and Planetary Science Conference, Lunar and Planetary Institute, Houston.

Gorevan, S.P., Myrick, T., Davis, K., Chau, J.J., Bartlett, P., Mukherjee, S., Anderson, R., Squyres, S.W., Arvidson, R.E., Madsen, M.B., Bertelsen, P., Goetz, W., Binau, C.S., and Richter, L. (2003) Rock Abrasion Tool: Mars Exploration Rover mission. J Geophys Res 108, doi:10.1029/2003JE002061.

Grant, J.A., Golombek, M.P., Parker, T.J., Crisp, J.A., Squyres, S.W., and Weitz, C.M. (2004) Selecting landing sites for the 2003 Mars Exploration Rovers. Planet Space Sci 52:11-21.

Grant, J.A., Irwin, R.P., III, Grotzinger, J.P., Milliken, R.E., Tornabene, L.L., McEwen, A.S., Weitz, C.M., Squyres, S.W., 
Glotch, T.D., and Thomson, B.J. (2008) HiRISE imaging of impact megabreccia and sub-meter aqueous strata in Holden Crater, Mars, Geology 36:195-198.

Grant, J.A., Irwin, R.P., Wilson, S.A., Buczkowski, D., and Siebach, K. (2010) A lake in Uzboi Vallis and implications for Late Noachian-Early Hesperian climate on Mars. Icarus 212: 100-122.

Grant, J.A., Golombek, M.P., Grotzinger, J.P., Wilson, S.A., Watkins, M.M., Vasavada, A.R., Griffes, J.L., and Parker, T.J. (2011) The science process for selecting the landing site for the 2011 Mars Science Laboratory. Planet Space Sci 59:11141127.

Greeley, R., Foing, B.H., McSween, H.Y., Jr., Neukum, G., Pinet, P., van Kan, M., Werner, S.C., Williams, D.A., and Zegers, T.E. (2005) Fluid lava flows in Gusev Crater, Mars. J Geophys Res 110, doi:10.1029/2005JE002401.

Grotzinger, J., Bell, J., III, Herkenhoff, K., Johnson, J., Knoll, A., McCartney, E., McLennan, S., Metz, J., Moore, J., Squyres, S., Sullivan, R., Ahronson, O., Arvidson, R., Joliff, B., Golombek, M., Lewis, K., Parker, T., and Soderblom, J. (2006) Sedimentary textures formed by aqueous processes, Erebus Crater, Meridiani Planum, Mars. Geology 34:1085-1088.

Grotzinger, J., Beaty, D., Dromart, G., Gupta, S., Harris, M., Hurowitz, J., Kocurek, G., McLennan, S., Milliken, R., Ori, G.G., and Sumner, D. (2011) Mars sedimentary geology: key concepts and outstanding questions. Astrobiology 11:77-87.

Grotzinger, J.P., Arvidson, R.E., Bell, J.F., III, Calvin, W., Clark, B.C., Fike, D.A., Golombek, M., Greeley, R., Haldemann, A., Herkenhoff, K.E., Jolliff, B.L., Knoll, A.H., Malin, M., McLennan, S.M., Parker, T., Soderblom, L., Sohl-Dickstein, J.N., Squyres, S.W., Tosca, N.J., and Watters, W.A. (2005) Stratigraphy and sedimentology of a dry to wet eolian depositional system, Burns Formation, Meridiani Planum, Mars. Earth Planet Sci Lett 240: $11-72$.

Hamilton, V.E., Christensen, P.R., McSween, H.Y., and Bandfield, J.L. (2003) Searching for the source regions of martian meteorites using MGS TES: integrating martian meteorites into the global distribution of igneous materials on Mars. Meteorit Planet Sci 38:871-885.

Hartmann, W.K. and Neukum, G. (2001) Cratering chronology and the evolution of Mars. Space Sci Rev 96:165-194.

Haskin, L.A., Wang, A., Jolliff, B.L., McSween, H.Y., Clark, B.C., Des Marais, D.J., McLennan, S.M., Tosca, N.J., Hurowitz, J.A., Farmer, J.D., Yen, A., Squyres, S.W., Arvidson, R.E., Klingelhöfer, G., Schröder, C., de Souza, P.A., Jr., Ming, D.W., Gellert, R., Zipfel, J., Brückner, J., Bell, J.F., III, Herkenhoff, K., Christensen, P.R., Ruff, S., Blaney, D., Gorevan, S., Cabrol, N.A., Crumpler, L., Grant, J., and Soderblom, L. (2005) Water alteration of rocks and soils on Mars at the Spirit rover site in Gusev Crater. Nature 436:66-69.

Head, J.W., Mustard, J.F., Kreslavsky, M.A., Milliken, R.E., and Marchant, D.R. (2003) Recent ice ages on Mars. Nature 426:797-802.

Hecht, M.H., Kounaves, S.P., Quinn, R.C., West, S.J., Young, S.M.M., Ming, D.W., Catling, D.C., Clark, B.C., Boynton, W.V., Hoffman, J., DeFlores, L.P., Gospodinova, K., Kapit, J., and Smith, P.H. (2009) Detection of perchlorate and the soluble chemistry of martian soil at the Phoenix lander site. Science 325:64-67.

Heiken, G.H., Vaniman, D.T., and French, B.M., editors. (1991) Lunar Sourcebook: A User's Guide to the Moon, Cambridge University Press, Cambridge, UK.

Hemming, S.R. (2004) Heinrich events: massive late Pleistocene detritus layers of the North Atlantic and their global climate imprint. Rev Geophys 42, doi:10.1029/ 2003RG000128.

Herkenhoff, K.E., Squyres, S.W., Arvidson, R., Bass, D.S., Bell, J.F., III, Bertelsen, P., Ehlmann, B.L., Farrand, W., Gaddis, L., Greeley, R., Grotzinger, J., Hayes, A.G., Hviid, S.F., Johnson, J.R., Jolliff, B., Kinch, K.M., Knoll, A.H., Madsen, M.B., Maki, J.N., McLennan, S.M., McSween, H.Y., Ming, D.W., Rice, J.W., Jr., Richter, L., Sims, M., Smith, P.H., Soderblom, L.A., Spanovich, N., Sullivan, R., Thompson, S., Wdowiak, T., Weitz, C., and Whelley, P. (2004) Evidence from Opportunity's Microscopic Imager for water on Meridiani Planum. Science 306:1727-1730.

Howard, A.D. (2007) Simulating the development of martian highland landscapes through the interaction of impact cratering, fluvial erosion, and variable hydrologic forcing. Geomorphology 91:332-363.

Howard, A.D., Moore, J.M., and Irwin, R.P., III. (2005) An intense terminal epoch of widespread fluvial activity on early Mars: 1. Valley network incision and associated deposits. J Geophys Res 110, doi:10.1029/2005JE002459.

Hurowitz, J.A., McLennan, S.M., Tosca, N.J., Arvidson, R.E., Michalski, J.R., Ming, D.W., Schröder, C., and Squyres, S.W. (2006) In situ and experimental evidence for acidic weathering of rocks and soils on Mars. J Geophys Res 111, doi:10.1029/ 2005JE002515.

Hynek, B.M. and Phillips, R.J. (2003) New data reveal mature, integrated drainage systems on Mars indicative of past precipitation. Geology 31:757-760.

iMars Working Group. (2008) Preliminary planning for an international Mars sample return mission: report of the International Mars Architecture for the Return of Samples (iMARS) working group, white paper, posted July 2008 by the Mars Exploration Program Analysis Group (MEPAG). Available online at http://mepag.jpl.nasa.gov/reports/iMARS_Final Report.pdf.

Jakosky, B.M. and Millour, E. (2011) The 2013 Mars Atmosphere and Volatile EvolutioN (MAVEN) Mission to Mars. In Fourth International Workship on the Mars Atmosphere: Modelling and Observation, Paris.

Jakosky, B.M., Nealson, K.H., Bakermans, C., Ley, R.E., and Mellon, M.T. (2003) Subfreezing activity of microorganisms and the potential habitability of Mars' polar regions. Astrobiology 3:343-350.

Jehlička, J., Edwards, H.G.M., and, Vítek, P. (2009) Assessment of Raman spectroscopy as a tool for the non-destructive identification of organic minerals and biomolecules for Mars studies. Planet Space Sci 57:606-613.

Johnson, A.P. and Pratt, L.M. (2010) Metal-catalyzed degradation and racemization of amino acids in iron sulfate brines under simulated martian surface conditions. Icarus 207: 124-132.

Jones, J.H. (2007) The edge of wetness: the case for dry magmatism on Mars, II [abstract 2006]. In Workshop on Water in Planetary Basalts, Lunar and Planetary Institute, Houston.

Kasting, J.F. (1991) $\mathrm{CO}_{2}$ condensation and the climate of early Mars. Icarus 94:1-13.

Kelley, D.S., Karson, J.A., Früh-Green, G.L., Yoerger, D.R., Shank, T.M., Butterfield, D.A., Hayes, J.M., Schrenk, M.O., Olson, E.J., Proskurowski, G., Jakuba, M., Bradley, A., Larson, B., Ludwig, K., Glickson, D., Buckman, K., Bradley, A.S., Brazelton, W.J., Roe, K., Elend, M.J., Delacour, A., Bernasconi, S.M., Lilley, M.D., Baross, J.A., Summons, R.E., and Sylva, S.P. (2005) A serpentinite-hosted ecosystem: the Lost City hydrothermal field. Science 307:1428-1434. 
Khan, A. and Connolly, J.A.D. (2008) Constraining the composition and thermal state of Mars from inversion of geophysical data. J Geophys Res 113, doi:10.1029/2007JE002996.

Klingelhöfer, G., Morris, R.V., Bernhardt, B., Schröder, C., Rodionov, D.S., de Souza, P.A., Jr., Yen, A., Gellert, R., Evlanov, E.N., Zubkov, B., Foh, J., Bonnes, U., Kankeleit, E., Gütlich, P., Ming, D.W., Renz, F., Wdowiak, T., Squyres, S.W., and Arvidson, R.E. (2004) Jarosite and hematite at Meridiani Planum from Opportunity's Mössbauer spectrometer. Science 306:1740-1745.

Kminek, G. and Bada, J.L. (2006) The effect of ionizing radiation on the preservation of amino acids on Mars. Earth Planet Sci Lett 245:1-5.

Knoll, A.H., Jolliff, B.L., Farrand, W.H., Bell, J.F., III, Clark, B.C., Gellert, R., Golombek, M.P., Grotzinger, J.P., Herkenhoff, K.E., Johnson, J.R., McLennan, S.M., Morris, R., Squyres, S.W., Sullivan, R., Tosca, N.J., Yen, A., and Learner, Z. (2008) Veneers, rinds, and fracture fills: relatively late alteration of sedimentary rocks at Meridiani Planum, Mars. J Geophys Res 113, doi:10.1029/2007JE002949.

Kuhn, W.R. and Atreya, S.K. (1979) Ammonia photolysis and the greenhouse effect in the primordial atmosphere of the Earth. Icarus 37:207-213.

Lang, N.P., Tornabene, L.L., McSween, H.Y., and Christensen, P.R. (2009) Tharsis-sourced relatively dust-free lavas and their possible relationship to martian meteorites. Journal of Volcanology and Geothermal Research 185:103-115.

Lapen, T.J., Righter, M., Brandon, A.D., Debaille, V., Beard, B.L., Shafer, J.T., and Peslier, A.H. (2010) A younger age for ALH84001 and its geochemical link to shergottite sources in Mars. Science 328:347-351.

Laskar, J., Levrard, B., and Mustard, J.F. (2002) Orbital forcing of the martian polar layered deposits. Nature 419:375-377.

Leshin, L.A. and Vicenzi, E. (2006) Aqueous processes recorded by martian meteorites: analyzing martian water on Earth. Elements 2:157-162.

Levine, J.S. (1985) The Photochemistry of Atmospheres: Earth, the Other Planets, and Comets, Academic Press, Orlando, FL.

Lewis, K.W., Aharonson, O., Grotzinger, J.P., Kirk, R.L., McEwen, A.S., and Suer, T.A. (2008a) Quasi-periodic bedding in the sedimentary rock record of Mars. Science 322:1532-1535.

Lewis, K.W., Aharonson, O., Grotzinger, J.P., Squyres, S.W., Bell, J.F., III, Crumpler, L.S., and Schmidt, M.E. (2008b) Structure and stratigraphy of Home Plate from the Spirit Mars Exploration Rover. J Geophys Res 113, doi:10.1029/2007JE003025.

Lillis, R.J., Frey, H.V., Manga, M., Mitchell, D.L., Lin, R.P., Acuña, M.H., and Bougher, S.W. (2008) An improved crustal magnetic field map of Mars from electron reflectometry: highland volcano magmatic history and the end of the martian dynamo. Icarus 194:575-596.

Lin, L.H., Wang, P.L., Rumble, D., Lippmann-Pipke, J., Boice, E., Pratt, L.M., Sherwood Lollar, B., Brodie, E.L., Hazen, T.C., Andersen, G.L., DeSantis, T.Z., Moser, D.P., Kershaw, D., and Onstott, T.C. (2006) Long-term sustainability of a high-energy, low-diversity crustal biome. Science 314:479-482.

Loizeau, D., Mangold, N., Poulet, F. Bibring, J.-P., Gendrin, A., Ansan, V., Gomez, C., Gondet, B., Langevin, Y., Masson, P., and Neukum, G. (2007) Phyllosilicates in the Mawrth Vallis region of Mars. J Geophys Res 112, doi:10.1029/2006JE002877.

Loizeau, D., Mangold, N., Poulet, F., Ansan, V., Hauber, E., Bibring, J.-P., Gondet, B., Langevin, Y., Masson, P., and Neukum, G (2010) Stratigraphy in the Mawrth Vallis region through OMEGA, HRSC color imagery and DTM. Icarus 205:396-418.
Lowe, D.R. (1983) Restricted shallow-water sedimentation of early Archean stromatolitic and evaporitic strata of the Strelley Pool Chert, Pilbara Block, Western Australia. Precambrian Res 19:239-283.

Macke, R.J., Britt, D.T., and Consolmagno, G.J. (2011) Density, porosity, and magnetic susceptibility of achondritic meteorites. Meteorit Planet Sci 46:311-326.

MacPherson, G. and the Mars Sample Return Science Steering Group II (2005) The first Mars surface-sample return mission: Revised science considerations in light of the 2004 MER results. Unpublished white paper, 68 pp, Appendix III of Science Properties for Mars Sample Return; posted March 2008 by the Mars Exploration Program Analysis Group (MEPAG) at http://mepag.jpl.nasa.gov/reports/ndsag.html.

Mahaffy, P.R., Beaty, D.W., Anderson, M., Aveni, G., Bada, J., Clemett, S., Des Marais, D., Douglas, S., Dworkin, J., Kern, R., Papanastassiou, D., Palluconi, F., Simmonds, J., Steele, A., Waite, H., and Zent, A. (2004) Science priorities related to the organic contamination of Martian landers. Unpublished white paper, 32 pp; posted November 2004 by the Mars Exploration Program Analysis Group (MEPAG) at http://mepag.jpl.nasa .gov/reports/index.html.

Malin, M.C. and Edgett, K.S. (2003) Evidence for persistent flow and aqueous sedimentation on early Mars. Science 302:19311934.

Mangold, N., Poulet, F., Mustard, J.F., Bibring, J.-P., Gondet, B., Langevin, Y., Ansan, V., Masson, Ph., Fassett, C., Head, J.W., III, Hoffmann, H., and Neukum, G. (2007) Mineralogy of the Nili Fossae region with OMEGA/Mars Express data: 2. Aqueous alteration of the crust. J Geophys Res 112, doi:10.1029/ 2006JE002835.

Marinova, M.M., Aharonson, O., and Asphaug, E. (2011) Geophysical consequences of planetary-scale impacts into a Marslike planet. Icarus 211:960-985.

Mathew, K.J. and Marti, K. (2002) Martian atmospheric and interior volatiles in the meteorite Nakhla. Earth Planet Sci Lett 199:7-20.

Mattingly, R. and May, L. (2011) Mars sample return as a campaign. IEEEAC Paper \#1805, Version 1, updated October 12, 2010, Institute of Electrical and Electronics Engineers (IEEE), Piscataway, NJ.

McCoy, T.J., Corrigan, C.M., and Herd, C.D.K. (2011) Combining meteorites and missions to explore Mars. Proc Natl Acad Sci USA 108:19159-19164.

McCubbin, F.M., Smirnov, A., Nekvasil, H., Wang, J., Hauri, E., and Lindsley, D.H. (2010) Hydrous magmatism on Mars: a source of water for the surface and subsurface during the Amazonian. Earth Planet Sci Lett 292:132-138.

McEwen, A.S., Ojha, L., Dundas, C.M., Mattson, S.S., Byrne, S., Wray, J.J., Cull, S.C., Murchie, S.L., Thomas, N., and Gulick, V.C. (2011) Seasonal flows on warm martian slopes. Science 333:740-743.

McKay, D.S., Gibson, E.K., Jr., Thomas-Keprta, K.L., Vali, H., Romanek, C.S., Clemett, S.J., Chillier, X.D., Maechling, C.R., and Zare, R.N. (1996) Search for past life on Mars: possible relic biogenic activity in martian meteorite ALH84001. Science 273:924-930.

McLennan, S.M. and Grotzinger, J.P. (2008) The sedimentary rock cycle of Mars. In The Martian Surface: Composition, Mineralogy, and Physical Properties, edited by J. Bell, III, Cambridge University Press, Cambridge, pp 541-577.

McLennan, S.M., Bell, J.F., III, Calvin, W.M., Christensen, P.R., Clark, B.C., de Souza, P.A., Farmer, J., Farrand, W.H., Fike, D.A., Gellert, R., Ghosh, A., Glotch, T.D., Grotzinger, J.P., Hahn, B., Herkenhoff, K.E., Hurowitz, J.A., Johnson, J.R., 
Johnson, S.S., Jolliff, B., Klingelhöfer, G., Knoll, A.H., Learner, Z., Malin, M.C., McSween, H.Y., Jr., Pocock, J., Ruff, S.W., Soderblom, L.A., Squyres, S.W., Tosca, N.J., Watters, W.A., Wyatt, M.B., and Yen, A. (2005) Provenance and diagenesis of the evaporite-bearing Burns Formation, Meridiani Planum, Mars. Earth Planet Sci Lett 240:95-121.

McSween, H. (1994) What we have learned about Mars from SNC meteorites. Meteoritics 29:757-779.

McSween, H.Y., Grove, T.L., Lentz, R.C., Dann, J.C., Holzheid, A.H., Riciputi, L.R., and Ryan, J.G. (2001) Geochemical evidence for magmatic water within Mars from pyroxenes in the Shergotty meteorite. Nature 409:487-490.

McSween, H.Y., Arvidson, R.E., Bell, J.F., Blaney, D., Cabrol, N.A., Christensen, P.R., Clark, B.C., Crisp, J.A., Crumpler, L.S., Des Marais, D.J., Farmer, J.D., Gellert, R., Ghosh, A., Gorevan, S., Graff, T., Grant, J., Haskin, L.A., Herkenhoff, K.E., Johnson, J.R., Jolliff, B.L., Klingelhoefer, G., Knudson, A.T., McLennan, S., Milam, K.A., Moersch, J.E., Morris, R.V., Rieder, R., Ruff, S.W., de Souza, P.A., Squyres, S.W., Wanke, H., Wang, A., Wyatt, M.B., Yen, A., and Zipfel, J. (2004) Basaltic rocks analyzed by the Spirit rover in Gusev Crater. Science 305:842-845.

McSween, H.Y., Ruff, S.W., Morris, R.V., Bell, J.F., III, Herkenhoff, K., Gellert, R., Stockstill, K.R., Tornabene, L.L., Squyres, S.W., Crisp, J.A., Christensen, P.R., McCoy, T.J., Mittlefehldt, D.W., and Schmidt, M. (2006) Alkaline volcanic rocks from the Columbia Hills, Gusev Crater, Mars. J Geophys Res 111, doi:10.1029/2006JE002698.

McSween, H.Y., Taylor, G.J., and Wyatt, M.B. (2009) Elemental composition of the martian crust. Science 324:736-739.

McSween, H.Y., Jr., McGlynn, I.O., and Rogers, A.D. (2010) Determining the modal mineralogy of martian soils. J Geophys Res 115, doi:10.1029/2010JE003582.

Melosh, H.J. (1989) Impact Cratering: a Geologic Process, research supported by NASA, Oxford Monographs on Geology and Geophysics, No. 11, Oxford University Press, New York.

Melosh, H.J. and Vickery, A.M. (1989) Impact erosion of the primordial atmosphere of Mars. Nature 338:487-489.

MEPAG. (2002) Groundbreaking MSR: science requirements and cost estimates for a first Mars surface-sample return mission, white paper posted October 2002 by the Mars Exploration Program Analysis Group (MEPAG). Available online at http://mepag.jpl.nasa.gov/reports/index.html.

MEPAG. (2010) Mars science goals, objectives, investigations, and priorities, white paper posted September 2010 by the Mars Exploration Program Analysis Group (MEPAG). Available online at http://mepag.jpl.nasa.gov/reports/index.html.

MEPAG 2R-iSAG. (2010) Two rovers on the same site on Mars, 2018: possibilities for cooperative science, white paper posted July 2010 by the Mars Exploration Program Analysis Group (MEPAG). Available online at http://mepag.jpl.nasa.gov/ reports/2R-iSAG_final.pdf.

MEPAG E2E-iSAG. (2011) Planning for Mars returned sample science: Final report of the MSR End-to-End International Science Analysis Group (E2E-iSAG), 101 pp; posted December 2011 by the Mars Exploration Program Analysis Group (MEPAG) at http://mepag.jpl.nasa.gov/reports/

MEPAG MRR-SAG. (2010) The Mars Astrobiology ExplorerCacher (MAX-C): a potential rover mission for 2018: final report of the Mars Mid-Range Rover Science Analysis Group. Astrobiology 10:127-163.

MEPAG ND-SAG. (2008) Science priorities for Mars sample return. Astrobiology 8:489-535.

Michalski, J.R., Bibring, J.-P., Poulet, F., Loizeau, D., Mangold, N., Dobrea, E.N., Bishop, J.L., Wray, J.J., McKeown, N.K.,
Parente, M., Hauber, E., Altieri, F., Carrozzo, F.G., and Niles, P.B. (2010) The Mawrth Vallis Region of Mars: a potential landing site for the Mars Science Laboratory (MSL) mission. Astrobiology 10:687-703.

Ming, D.W., Morris, R.V., Woida, R., Sutter, B., Lauer, H.V., Shinohara, C., Golden, D.C., Boynton, W.V., Arvidson, R.E., Stewart, R.L., Tamppari, L.K., Gross, M., and Smith, P. (2008) Mars 2007 Phoenix Scout mission Organic Free Blank: method to distinguish Mars organics from terrestrial organics. J Geophys Res 113, doi:10.1029/2007JE003061.

Moore, H.J. (1991) Estimates of Some Physical/Mechanical Properties of Martian Rocks and Soil-Like Materials, USGS Open File Report 91-568, U.S. Geological Survey, Menlo Park, CA.

Morris, R.V., Ruff, S.W., Gellert, R., Ming, D.W., Arvidson, R.E., Clark, B.C., Golden, D.C., Siebach, K., Klingelhöfer, G., Schröder, C., Fleischer, I., Yen, A.S., and Squyres, S.W. (2010) Identification of carbonate-rich outcrops on Mars by the Spirit rover. Science 329:421-424.

Murchie, S., Kirkland, L., Erard, S., Mustard, J., and Robinson, M. (2000) Near-infrared spectral variations of martian surface materials from ISM imaging spectrometer data. Icarus 147:444-471.

Murchie, S.L., Mustard, J.F., Ehlmann, B.L., Milliken, R.E., Bishop, J.L., McKeown, N.K., Noe Dobrea, E.Z., Seelos, F.P., Buczkowski, D.L., Wiseman, S.M., Arvidson, R.E., Wray, J.J., Swayze, G., Clark, R.N., Des Marais, D.J., McEwen, A.S., and Bibring, J.-P. (2009) A synthesis of martian aqueous mineralogy after 1 Mars year of observations from the Mars Reconnaissance Orbiter. J Geophys Res 114, doi:10.1029/2009 JE003342.

Musselwhite, D.S., Dalton, H.A., Kiefer, W.S., and Treiman, A.H. (2006) Experimental petrology of the basaltic shergottite Yamato-980459: implications for the thermal structure of the martian mantle. Meteorit Planet Sci 41:1271-1290.

Mustard, J.F., Poulet, F., Head, J.W., Mangold, N., Bibring, J.-P., Pelkey, S.M., Fassett, C.I., Langevin, Y., and Neukum, G. (2007) Mineralogy of the Nili Fossae region with OMEGA/ Mars Express data: 1. Ancient impact melt in the Isidis Basin and implications for the transition from the Noachian to Hesperian. J Geophys Res 112, doi:10.1029/2006JE002834.

Mustard, J.F., Murchie, S.L., Pelkey, S.M., Ehlmann, B.L., Milliken, R.E., Grant, J.A., Bibring, J.-P., Poulet, F., Bishop, J., Noe Dobrea, E., Roach, L., Seelos, F., Arvidson, R.E., Wiseman, S., Green, R., Hash, C., Humm, D., Malaret, E., McGovern, J.A., Seelos, K., Clancy, T., Clark, R., Des Marais, D., Izenberg, N., Knudson, A., Langevin, Y., Martin, T., McGuire, P., Morris, R., Robinson, M., Roush, T., Smith, M., Swayze, G., Taylor, H., Titus, T., and Wolff, M. (2008) Hydrated silicate minerals on Mars observed by the Mars Reconnaissance Orbiter CRISM instrument. Nature 454:305-309.

Mustard, J.F., Ehlmann, B.L., Murchie, S.L., Poulet, F., Mangold, N., Head, J.W., Bibring, J.-P., and Roach, L.H. (2009) Composition, morphology, and stratigraphy of Noachian crust around the Isidis Basin. J Geophys Res 114, doi:10.1029/ 2009JE003349.

Mysen, B.O., Virgo, D., Popp, R.K., and Bertka, C.M. (1998) The role of $\mathrm{H}_{2} \mathrm{O}$ in martian magmatic systems. Am Mineral 83: 942-946.

NASA. (1995) An Exobiology Strategy for Mars Exploration, NASA SP-530, National Aeronautics and Space Administration, Washington DC.

Neukum, G., Jaumann, R., Hoffmann, H., Hauber, E., Head, J.W., Basilevsky, A.T., Ivanov, B.A., Werner, S.C., van Gasselt, S., Murray, J.B., McCord, T.B., and the HRSC Co-Investigator 
team. (2004) Recent and episodic volcanic and glacial activity on Mars revealed by the High Resolution Stereo Camera. Nature 432:971-979.

Niles, P.B., Zolotov, M.Yu., and Leshin, L.A. (2009) Insights into the formation of Fe-and Mg-rich aqueous solutions on early Mars provided by the ALH 84001 carbonates. Earth Planet Sci Lett 286:122-130.

Niles, P.B., Boynton, W.V., Hoffman, J.H., Ming, D.W., and Hamara, D. (2010) Stable isotope measurements of Martian atmospheric $\mathrm{CO}_{2}$ at the Phoenix landing site. Science 329:1334-1337.

Nisbet, E., Zahnle, K., Gerasimov, M.V., Helbert, J., Jaumann, R., Hofmann, B.A., Benzerara, K., and Westall, F. (2007) Creating habitable zones, at all scales, from planets to mud microhabitats, on Earth and on Mars. In Geology and Habitability of Terrestrial Planets, Space Series of ISSI, Vol. 24, edited by K.E. Fishbaugh, P. Lognonné, F. Raulin, D.J. Des Marais, and O. Korablev, Springer, New York, pp 79-121.

NRC. (1978) Strategy for the Exploration of the Inner Planets: 19771987, The National Academy of Sciences, Washington DC.

NRC. (1990a) Update to Strategy for Exploration of the Inner Planets, The National Academies Press, Washington DC.

NRC. (1990b) International Cooperation for Mars Exploration and Sample Return, The National Academies Press, Washington DC.

NRC. (1994) An Integrated Strategy for the Planetary Sciences: 1995-2010, The National Academies Press, Washington DC.

NRC. (1996) Review of NASA's Planned Mars Program, The National Academies Press, Washington DC.

NRC. (2003) Assessment of Mars Science and Mission Priorities, The National Academies Press, Washington DC.

NRC. (2006) Assessment of NASA's Mars Architecture 2007-2016, National Academies Press, Washington DC.

NRC. (2007) An Astrobiology Strategy for the Exploration of Mars, The National Academies Press, Washington DC.

NRC. (2011) Vision and Voyages for Planetary Science in the Decade 2013-2022, The National Academies Press, Washington DC.

NRC. (2009) Assessment of Planetary Protection Requirements for Mars Sample Return Missions, The National Academies Press, Washington DC.

Nyquist, L.E., Bogard, D.D., Shih, C.-Y., Greshake, A., Stöffler, D., and Eugster, O. (2001) Ages and geologic histories of martian meteorites. Space Sci Rev 96:105-164

Onstott, T.C., Lin, L.-H., Davidson, M., Mislowack, B., Borcsik, M., Hall, J., Slater, G., Ward, J., Lollar, B., Lippmann-Pipke, J., Boice, E., Pratt, L., Pfiffner, S., Moser, D., Gihring, T., Kieft, T., Phelps, T., Vanheerden, E., Litthaur, D., Deflaun, M., Rothmel, R., Wanger, G., and Southam, G. (2006) The origin and age of biogeochemical trends in deep fracture water of the Witwatersrand Basin, South Africa. Geomicrobiol J 23:369-414.

Orange, F., Westall, F., Disnar, J.R., Prieur, D., Bienvenu, N., Le Romancer, M., and Défarge, Ch. (2009) Experimental silicification of the extremophilic archaea Pyrococcus abyssi and Methanocaldococcus jannaschii: applications in the search for evidence of life in early Earth and extraterrestrial rocks. Geobiology 7:403-418.

Osterloo, M.M., Hamilton, V.E., Bandfield, J.L., Glotch, T.D., Baldridge, A.M., Christensen, P.R., Tornabene, L.L., and Anderson, F.S. (2008) Chloride-bearing materials in the southern highlands of Mars. Science 319:1651-1654.

Papike, J.J., Karner, J.M., Shearer, C.K., and Burger, P.V. (2009) Silicate mineralogy of martian meteorites. Geochim Cosmochim Acta 73:7443-7485.

Parnell, J., Cullen, D., Sims, M.R., Bowden, S., Cockell, C.S., Court, R., Ehrenfreund, P., Gaubert, F., Grant, W., Parro, V., Rohmer, M., Sephton, M., Stan-Lotter, H., Steele, A., Toporski,
J., and Vago, J. (2007) Searching for life on Mars: selection of molecular targets for ESA's aurora ExoMars mission. Astrobiology 7:578-604.

Pepin, R.O. (1991) On the origin and early evolution of terrestrial planet atmospheres and meteoritic volatiles. Icarus 92:2-79.

Pepin, R.O. (1994) Evolution of the martian atmosphere. Icarus 111:289-304

Pepin, R.O. (2000) On the isotopic composition of primordial xenon in terrestrial planet atmospheres. Space Sci Rev 92:371-395.

Pepin, R.O. (2006) Atmospheres on the terrestrial planets: clues to origin and evolution. Earth Planet Sci Lett 252:1-14.

Perl, S.M., McLennan, S.M., Grotzinger, J.P., Herkenhoff, K.E., and the Athena Science team. (2007) Volumes and orientation of secondary porosity in the Burns Formation, Meridiani Planum, Mars [abstract 2226]. In 38 ${ }^{\text {th }}$ Lunar and Planetary Institute Science Conference Abstracts, Lunar and Planetary Institute, Houston.

Pettijohn, F.J. (1984) Memoirs of an Unrepentant Field Geologist, University of Chicago Press, Chicago.

Pondrelli, M., Rossi, A.P., Marinangeli, L., Hauber, E., Gwinner, K., Baliva, A., and di Lorenzo, S. (2008) Evolution and depositional environments of the Eberswalde fan delta, Mars. Icarus 197:429-451.

Poulet, F., Bibring, J.-P., Mustard, J.F., Gendrin, A., Mangold, N., Langevin, Y., Arvidson, R.E., Gondet, B., and Gomez, C. (2005) Phyllosilicates on Mars and implications for early martian climate. Nature 438:623-627.

Price, H., Cramer, K., Doudrick, S., Lee, W., Matijevic, J., Weinstein, S., Lam-Trong, T., Marsal, O., and Mitcheltree, R. (2000) Mars Sample Return spacecraft systems architecture. In 2000 IEEE Aerospace Conference Proceedings, Institute of Electrical and Electronics Engineers (IEEE), Piscataway, NJ, doi:10.1109/AERO.2000.879302.

Proskurowski, G., Lilley, M.D., Seewald, J.S., Früh-Green, G.L., Olson, E.J., Lupton, J.E., Sylva, S.P., and Kelley, D.S. (2008) Abiogenic hydrocarbon production at Lost City hydrothermal field. Science 319:604-607.

Quinn, R., Zent, A.P., and McKay, C.P. (2006) The photochemical stability of carbonates on Mars. Astrobiology 6:581-591.

Rogers, A.D. (2010) Crustal compositions exposed by impact craters in the Tyrrhena Terra region of Mars: considerations for Noachian environments. Earth Planet Sci Lett 301:353-364.

Rogers, A.D. and Bandfield, J.L. (2009) Mineralogical characterization of Mars Science Laboratory candidate landing sites from THEMIS and TES data. Icarus 203:437-453.

Rouchy, J.-M. and Monty, C.L.V. (1981) Stromatolites and cryptalgal laminites associated with Messinian gypsum of Cyprus. In Phanerozoic Stromatolites, edited by C.L.V. Monty, Springer, Berlin, pp 155-180.

Ruff, S.W. (2004) Spectral evidence for zeolite in the dust on Mars. Icarus 168:131-143.

Ruff, S.W., Christensen, P.R., Blaney, D.L., Farrand, W.H., Johnson, J.R., Michalski, J.R., Moersch, J.E., Wright, S.P., and Squyres, S.W. (2006) The rocks of Gusev Crater as viewed by the Mini-TES instrument. J Geophys Res 111, doi:10.1029/2006JE002747.

Ruff, S.W., Farmer, J.D., Calvin, W.M., Herkenhoff, K.E., Johnson, J.R., Morris, R.V., Rice, M.S., Arvidson, R.E., Bell, J.F., III, Christensen, P.R., and Squyres, S.W. (2011) Characteristics, distribution, origin, and significance of opaline silica observed by the Spirit rover in Gusev Crater, Mars. J Geophys Res 116, doi:10.1029/2010JE003767.

Rummel, J.D., Race, M.S., DeVincenzi, D.L., Schad, P.J., Stabekis, P.D., Viso, M., and Acevedo, S.E., editors. (2002) A Draft Test Protocol for Detecting Possible Biohazards in Martian Samples 
Returned to Earth, NASA/CP-2002-211842, National Aeronautics and Space Administration, Washington DC.

Schmidt, M.E. and McCoy, T.J. (2010) The evolution of a heterogeneous martian mantle: clues from $\mathrm{K}, \mathrm{P}, \mathrm{Ti}, \mathrm{Cr}$, and $\mathrm{Ni}$ variations in Gusev basalts and shergottite meteorites. Earth Planet Sci Lett 296:67-77.

Schopf, J.W. (2006) Fossil evidence of Archean life. Philos Trans $R$ Soc Lond, B, Biol Sci 361:869-885.

Schopf, J.W. and Packer, B.M. (1987) Early Archean (3.3-billion to 3.5-billion-year-old) microfossils from Warrawoona Group, Australia. Science 237:70-73.

Segura, T.L., Toon, O.B., Colaprete, A., and Zahnle, K. (2002) Environmental effects of large impacts on Mars. Science 298:1977-1980.

Sherwood Lollar, B., Lacrampe-Couloume, G., Slater, G.F., Ward, J., Moser, D.P., Gihring, T.M., Lin, L.-H., and Onstott, T.C. (2006) Unravelling abiogenic and biogenic sources of methane in the Earth's deep subsurface. Chem Geol 226: 328-339.

Smith, P.H., Tamppari, L.K., Arvidson, R.E., Bass, D., Blaney, D., Boynton, W.V., Carswell, A., Catling, D.C., Clark, B.C., Duck, T., DeJong, E., Fisher, D., Goetz, W., Gunnlaugsson, H.P., Hecht, M.H., Hipkin, V., Hoffman, J., Hviid, S.F., Keller, H.U., Kounaves, S.P., Lange, C.F., Lemmon, M.T., Madsen, M.B., Markiewicz, W.J., Marshall, J., McKay, C.P., Mellon, M.T., Ming, D.W., Morris, R.V., Pike, W.T., Renno, N., Staufer, U., Stoker, C., Taylor, P., Whiteway, J.A., and Zent, A.P. (2009) $\mathrm{H}_{2} \mathrm{O}$ at the Phoenix landing site. Science 325:58-61.

Squyres, S.W., Arvidson, R.E., Bell, J.F., III, Brückner, J., Cabrol, N.A., Calvin, W., Carr, M.H., Christensen, P.R., Clark, B.C., Crumpler, L., Des Marais, D.J., D'Uston, C., Economou, T., Farmer, J., Farrand, W., Folkner, W., Golombek, M., Gorevan, S., Grant, J.A., Greeley, R., Grotzinger, J., Haskin, L., Herkenhoff, K.E., Hviid, S., Johnson, J., Klingelhöfer, G., Knoll, A., Landis, G., Lemmon, M., Li, R., Madsen, M.B., Malin, M.C., McLennan, S.M., McSween, H.Y., Ming, D.W., Moersch, J., Morris, R.V., Parker, T., Rice, J.W., Jr., Richter, L., Rieder, R., Sims, M., Smith, M., Smith, P., Soderblom, L.A., Sullivan, R., Wänke, H., Wdowiak, T., Wolff, M., and Yen, A. (2004a) The Spirit Rover's Athena science investigation at Gusev Crater, Mars. Science 305:794-799.

Squyres, S.W., Arvidson, R.E., Bell, J.F., III, Brückner, J., Cabrol, N.A., Calvin, W., Carr, M.H., Christensen, P.R., Clark, B.C., Crumpler, L., Des Marais, D.J., d'Uston, C., Economou, T., Farmer, J., Farrand, W., Folkner, W., Golombek, M., Gorevan, S., Grant, J.A., Greeley, R., Grotzinger, J., Haskin, L., Herkenhoff, K.E., Hviid, S., Johnson, J., Klingelhöfer, G., Knoll, A.H., Landis, G., Lemmon, M., Li, R., Madsen, M.B., Malin, M.C., McLennan, S.M., McSween, H.Y., Ming, D.W., Moersch, J., Morris, R.V., Parker, T., Rice, J.W., Jr., Richter, L., Rieder, R., Sims, M., Smith, M., Smith, P., Soderblom, L.A., Sullivan, R., Wänke, H., Wdowiak, T., Wolff, M., and Yen, A. (2004b) The Opportunity Rover's Athena science investigation at Meridiani Planum, Mars. Science 306:1698-1703.

Squyres, S.W., Grotzinger, J.P., Arvidson, R.E., Bell, J.F., III, Calvin, W., Christensen, P.R., Clark, B.C., Crisp, J.A., Farrand, W.H., Herkenhoff, K.E., Johnson, J.R., Klingelhöfer, G., Knoll, A.H., McLennan, S.M., McSween, H.Y., Jr., Morris, R.V., Rice, J.W., Jr., Rieder, R., and Soderblom, L.A. (2004c) In situ evidence for an ancient aqueous environment at Meridiani Planum, Mars. Science 306:1709-1714.

Squyres, S.W., Arvidson, R.E., Blaney, D.L., Clark, B.C., Crumpler, L., Farrand, W.H., Gorevan, S., Herkenhoff, K.E., Hurowitz, J., Kusack, A., McSween, H.Y., Ming, D.W., Morris,
R.V., Ruff, S.W., Wang, A., and Yen, A. (2006) Rocks of the Columbia Hills. J Geophys Res 111, doi:10.1029/2005 JE002562.

Squyres, S.W., Aharonson, O., Clark, B.C., Cohen, B.A., Crumpler, L., de Souza, P.A., Farrand, W.H., Gellert, R., Grant, J., Grotzinger, J.P., Haldemann, A.F., Johnson, J.R., Klingelhöfer, G., Lewis, K.W., Li, R., McCoy, T., McEwen, A.S., McSween, H.Y., Ming, D.W., Moore, J.M., Morris, R.V., Parker, T.J., Rice, J.W., Jr., Ruff, S., Schmidt, M., Schröder, C., Soderblom, L.A., and Yen, A. (2007) Pyroclastic activity at Home Plate in Gusev Crater, Mars. Science 316:738-742.

Squyres, S.W., Arvidson, R.E., Ruff, S., Gellert, R., Morris, R.V., Ming, D.W., Crumpler, L., Farmer, J.D., Des Marais, D.J., Yen, A., McLennan, S.M., Calvin, W., Bell, J.F., III, Clark, B.C., Wang, A., McCoy, T.J., Schmidt, M.E., and de Souza, P.A., Jr. (2008) Detection of silica-rich deposits on Mars. Science 320:1063-1067.

Stalport, F., Coll, P., Szopa, C., Cottin, H., and Raulin, F. (2009) Investigating the photostability of carboxylic acids exposed to Mars surface ultraviolet radiation conditions. Astrobiology 9:543-549.

Steele, A., Fries, M., Amundsen, H.E.F., Mysen, B.O., Fogel, M.L., Schweizer, M., and Boctor, N.Z. (2007) Comprehensive imaging and Raman spectroscopy of carbonate globules from martian meteorite ALH 84001 and a terrestrial analogue from Svalbard. Meteorit Planet Sci 42:1549-1566.

Steltzner, A., Kipp, D., Chen, A., Burkhart, D., Guernsey, C., Mendeck, G., Mitcheltree, R., Powell, R., Rivellini, T., San Martin, M., and Way, D. (2006) Mars Science Laboratory entry, descent, and landing system. In 2006 IEEE Aerospace Conference, Institute of Electrical and Electronics Engineers (IEEE), Piscataway, NJ, doi:10.1109/AERO.2006 .1655796 .

Stevenson, D.J., Spohn, T., and Schubert, G. (1983) Magnetism and thermal evolution of the terrestrial planets. Icarus 54: 466-489.

Stolper, E. and McSween, H.Y. (1979) Petrology and origin of the shergottite meteorites. Geochim Cosmochim Acta 43: 1475-1498.

Sullivan, R., Banfield, D., Bell, J.F., Calvin, W., Fike, D., Golombek, M., Greeley, R., Grotzinger, J., Herkenhoff, K., Jerolmack, D., Malin, M., Ming, D., Soderblom, L.A., Squyres, S.W., Thompson, S., Watters, W.A., Weitz, C.M., and Yen, A. (2005) Aeolian processes at the Mars Exploration Rover Meridiani Planum landing site. Nature 436:58-61.

Sullivan, R., Arvidson, R., Bell, J.F., Gellert, R., Golombek, M., Greeley, R., Herkenhoff, K., Johnson, J., Thompson, S., Whelley, P., and Wray, J. (2008) Wind-driven particle mobility on Mars: insights from Mars Exploration Rover observations at "El Dorado" and surroundings at Gusev Crater. J Geophys Res 113, doi:10.1029/2008JE003101.

Summons, R.E., Amend, J.P., Bish, D., Buick, R., Cody, G.D., Des Marais, D.J., Dromart, G., Eigenbrode, J.L., Knoll, A.H., and Sumner, D.Y. (2011) Preservation of martian organic and environmental records: final report of the Mars Biosignature Working Group. Astrobiology 11:157-181.

Swindle, T.D. (2002) Martian noble gases. Reviews in Mineralogy and Geochemistry 47:171-190.

Swindle, T.D., Caffee, M.W., and Hohenberg, C.M. (1986) Xenon and other noble gases in shergottites. Geochim Cosmochim Acta 50:1001-1015.

Swindle, T.D., Thomas, C., Mousis, O., Lunine, J.I., and Picaud, S. (2009) Incorporation of argon, krypton and xenon into clathrates on Mars. Icarus 203:66-70. 
Thomas-Keprta, K.L., Clemett, S.J., McKay, D.S., Gibson, E.K., and Wentworth, S.J. (2009) Origins of magnetite nanocrystals in martian meteorite ALH84001. Geochim Cosmochim Acta 73:6631-6677.

Tosca, N.J., Knoll, A.H., and McLennan, S.M. (2008) Water activity and the challenge for life on early Mars. Science 320:1204-1207.

Treiman, A.H. and Essene, E.J. (2011) Chemical composition of magnetite in martian meteorite ALH 84001: revised appraisal from thermochemistry of phases in Fe-Mg-C-O. Geochim Cosmochim Acta 75:5324-5335.

United Nations. (1966) Treaty on Principles Governing the Activities of States in the Exploration and Use of Outer Space, Including the Moon and Other Celestial Bodies, Ref. 610 UNTS 205, Resolution 222 (XXI) of 19 December 1966, ratified on 27 January 1967.

Wacey, D., Kilburn, M.R., Saunders, M., Cliff, J., and Brasier, M.D. (2011) Microfossils of sulphur-metabolizing cells in 3.4billion-year-old rocks of Western Australia. Nature Geosci 4:698-702.

Walter, M.R. (1983) Archean stromatolites: evidence of the Earth's earliest benthos. In Earth's Earliest Biosphere: Its Origin and Evolution, edited by J.W. Schopf, Princeton University Press, Princeton, NJ, pp 187-213.

Walter, M.R. (1996) Ancient hydrothermal ecosystems on Earth: a new palaeobiological frontier. In Evolution of Hydrothermal Ecosystems on Earth (and Mars?), Ciba Foundation Symposium 202, edited by G.R. Bock and J.A. Goode, John Wiley and Sons, Chichester, UK, pp 112-130.

Walter, M.R. and Des Marais, D.J. (1993) Preservation of biological information in thermal spring deposits: developing a strategy for the search for fossil life on Mars. Icarus 101: 129-143.

Walton, E.L., Kelley, S.P., and Herd, C.D.K. (2008) Isotopic and petrographic evidence for young martian basalts. Geochim Cosmochim Acta 72:5819-5837.

Wang, A., Bell, J.F., III, Li, R., Johnson, J.R., Farrand, W.H., Cloutis, E.A., Arvidson, R.E., Crumpler, L., Squyres, S.W., McLennan, S.M., Herkenhoff, K.E., Ruff, S.W., Knudson, A.T., Chen, W., and Greenberger, R. (2008) Light-toned salty soils and coexisting Si-rich species discovered by the Mars Exploration Rover Spirit in Columbia Hills. J Geophys Res 113, doi:10.1029/2008JE003126.

Weiss, B.P., Fong, L.E., Vali, H., Lima, E.A., and Baudenbacher, F. (2008) Paleointensity of the ancient martian magnetic field. Geophys Res Lett 35, doi:10.1029/2008GL035585.

Westall, F., de Vries, S.T., Nijman, W., Rouchon, V., Orberger, B., Pearson, V., Watson, J., Verchovsky, A., Wright, I., Rouzaud, J.-N., Marchesini, D., and Severine, A. (2006) The 3.466 Ga "Kitty's Gap Chert," an Early Archaean microbial ecosystem. In Processes on the Early Earth, Geological Society of America Special Paper 405, edited by W.U. Reimold and R.L. Gibson, Geological Society of America, Boulder, CO, pp 105-131.

Wilson, L. and Head, J.W., III. (1994) Mars: review and analysis of volcanic eruption theory and relationships to observed landforms. Rev Geophys 32:221-263.
Yen, A.S., Kim, S.S., Hecht, M.H., Frant, M.S., and Murray, B. (2000) Evidence that the reactivity of the martian soil is due to superoxide ions. Science 289:1909-1912.

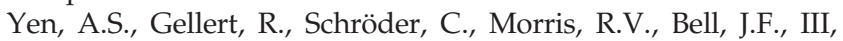
Knudson, A.T., Clark, B.C., Ming, D.W., Crisp, J.A., Arvidson, R.E., Blaney, D., Brückner, J., Christensen, P.R., Des Marais, D.J., de Souza, P.A., Jr., Economou, T.E., Ghosh, A., Hahn, B.C., Herkenhoff, K.E., Haskin, L.A., Hurowitz, J.A., Joliff, B.L., Johnson, J.R., Klingelhöfer, G., Madsen, M.B., McLennan, S.M., McSween, H.Y., Richter, L., Rieder, R., Rodionov, D., Soderblom, L., Squyres, S.W., Tosca, N.J., Wang, A., Wyatt, M., and Zipfel, J. (2005) An integrated view of the chemistry and mineralogy of martian soils. Nature 436:49-54.

Yen, A.S., Clark, B.C., Hurowitz, J.A., and the Athena Science team. (2006) Decomposition of organic compounds at the martian surface [abstract B13C-1114]. In American Geophysical Union, Fall Meeting 2006, American Geophysical Union, Washington DC.

Yen, A.S., Morris, R.V., Clark, B.C., Gellert, R., Knudson, A.T., Squyres, S., Mittlefehldt, D.W., Ming, D.W., Arvidson, R., McCoy, T., Schmidt, M., Hurowitz, J., Li, R., and Johnson, J.R. (2008) Hydrothermal processes at Gusev Crater: an evaluation of Paso Robles class soils. J Geophys Res 113, doi:10.1029/ 2007JE002978.

Younse, P., Collins, C., and Backes, P. (2010) A Sample Handling, Encapsulation, and Containerization subsystem concept for Mars sample caching missions. In International Planetary Probe Workshop 2010 (IPPW-7).

Address correspondence to: Scott McLennan

Department of Geosciences SUNY at Stony Brook Stony Brook, NY

USA

E-mail: scott.mclennan@sunysb.edu

Mark Sephton

Imperial College London

Department of Earth Science and Engineering

London

UK

E-mail: m.a.sephton@imperial.ac.uk

David Beaty

Mars Program Office

Jet Propulsion Laboratory

California Institute of Technology

Pasadena, CA

USA

E-mail: David.Beaty@jpl.nasa.gov

Submitted 8 December 2011

Accepted 11 January 2012 
This article has been cited by:

1. Grosch Eugene G., McLoughlin Nicola, Lanari Pierre, Erambert Muriel, Vidal Olivier. 2014. Microscale Mapping of Alteration Conditions and Potential Biosignatures in Basaltic-Ultramafic Rocks on Early Earth and Beyond. Astrobiology 14:3, 216-228. [Abstract] [Full Text HTML] [Full Text PDF] [Full Text PDF with Links] [Supplemental Material]

2. Abigail Allwood, David Beaty, Deborah Bass, Cassie Conley, Gerhard Kminek, Margaret Race, Steve Vance, Frances Westall. 2013. Conference Summary: Life Detection in Extraterrestrial Samples. Astrobiology 13:2, 203-216. [Citation] [Full Text HTML] [Full Text PDF] [Full Text PDF with Links] 